\title{
Multimodal multiscale analysis reveals flexible DNA contacts of bacterial nucleoprotein filaments
}

\author{
Beth A. Shen', Christine M. Hustmyer ${ }^{1}$, Daniel Roston ${ }^{1,2}$, Michael B. Wolfe ${ }^{1}$, Erik D. \\ Jessen $^{1}$, and Robert Landick ${ }^{1,3}$ \\ ${ }^{1}$ Department of Biochemistry, University of Wisconsin-Madison, Madison, WI 53706 \\ ${ }^{2}$ Current address: Department of Chemistry and Biochemistry, University of California San \\ Diego, La Jolla, CA 92093 \\ ${ }^{3}$ Department of Bacteriology, University of Wisconsin-Madison, Madison, WI 53706
}

*To whom correspondence should be addressed; Email: rlandick@wisc.edu

\section{Keywords:}

bacterial chromatin; H-NS; ChIP-seq; FeBABE; MD simulation; nucleoprotein; gene regulation

\section{Select Abbreviations:}

ChIP-seq, chromatin immunoprecipitation followed by high-throughput sequencing; DBD, DNA binding domain; DBP, DNA binding protein; FeBABE, Fe(III) (S)-1-(p-

Bromoacetamidobenzyl)ethylenediaminetetraacetate; Fe·EDTA-H-NS, H-NS derivatized with an $\mathrm{Fe}^{2+}$.EDTA nuclease; gDNA, genomic DNA; MD, molecular dynamics; NTD, N-terminal domain; $\bullet \mathrm{OH}$, hydroxyl radical; RNAP, RNA polymerase 


\section{ABSTRACT}

2 Gene silencing in bacteria is mediated by chromatin proteins, of which Escherichia coli $\mathrm{H}-\mathrm{NS}$ is

3 a paradigmatic example. H-NS forms nucleoprotein filaments with either one or two DNA

4 duplexes. However, the overall structures, arrangements of DNA-binding domains (DBDs),

5 positions of DBD-DNA contacts, and determinants of genomic distribution for these linear and

6 bridged filaments are uncertain. To connect H-NS structures that silence genes in vivo with

7 features elucidated in vitro, we developed a multimodal, multiscale analysis that combines a new

8 method for chromatin reconstitution and mapping (Nitro-seq), ChIP-seq, tethered-nuclease

9 mapping of DBD-DNA contacts (TEN-map), $\cdot \mathrm{OH}$ footprinting, molecular dynamics, and

10 bioinformatics. We find that DNA sequence principally governs H-NS-filament location with

11 indistinguishable sequence specificity in bridged or linear forms with or without the H-NS

12 modifiers StpA and Hha, and that DBD-DNA contacts vary in orientation and position with $\sim 10$ -

13 bp average spacing. Our results support a hemi-sequestration model of linear-to-bridged filament

14 switching. 


\section{INTRODUCTION}

16 Extensive study of nucleosome-based chromatin structure has provided broad insight into

17 mechanisms of chromatin-mediated gene regulation in eukaryotes (Cook and Marenduzzo, 2018;

18 Luger et al., 1997; Talbert et al., 2019). For bacteria, however, an analogous structural

19 understanding remains elusive in part due to the diverse and complex DNA-binding properties of

20 the multiple DNA-binding proteins (DBPs) that generate bacterial chromatin (Dame et al., 2020;

21 Grainger, 2016; Shen and Landick, 2019). The $\gamma$-proteobacterial histone-like nucleoid structuring

22 protein (H-NS; $15 \mathrm{kDa}$ monomer) provides a representative example of this complexity. H-NS,

23 which contains two oligomerization sites in its N-terminal domain (NTD) and a C-terminal DNA

24 binding domain (DBD), forms gene-silencing, oligomeric filaments on AT-rich DNA, notably on

25 pathogenicity operons (e.g. SPI-1 in Salmonella), surface-antigen operons (e.g. LPS biosynthesis

in Escherichia coli, and horizontally acquired genes (e.g. bgl operon in E. coli) (Gawade et al., 2020; Grainger et al., 2006; Kahramanoglou et al., 2011; Lucchini et al., 2006; Navarre et al., 2006; Sankar et al., 2009).

H-NS oligomerizes on DNA through head-head and tail-tail NTD contacts into filaments that adopt either linear or bridged conformations in which H-NS interacts with one or two

31 segments of duplex DNA, respectively (Arold et al., 2010; Gordon et al., 2011; Kotlajich et al., 2015; Liu et al., 2010; Ulissi et al., 2014) (Figure 1A). The DBD forms an AT-hook that binds the narrow minor groove of AT-rich DNA (Gordon et al., 2011). A flexible linker connecting the DBD and NTD enables DBD-DNA contacts. However, in some conditions, a subset of DBDs are proposed to interact with the NTD rather than DNA yielding a hemi-sequestered, linear conformation of H-NS-DNA filaments (Gao et al., 2017; Gulvady et al., 2018; van der Valk et al., 2017) (Figure 1A). Both linear and bridged filaments inhibit transcription initiation by RNA polymerase (RNAP) by occluding promoters but only bridged filaments inhibit transcript elongation by RNAP, which occurs via a topological trapping mechanism (Boudreau et al., 2018; Kotlajich et al., 2015; Lim et al., 2012) (Figure S1-1A). other DBPs controls the locations of the $\sim 0.2-20 \mathrm{~kb} \mathrm{H-NS}$ filaments that cover $\sim 15 \%$ of the $E$. 
coli K-12 genome is unclear (Kahramanoglou et al., 2011; Noom et al., 2007). Various DNA sequences, including a 10-bp high-affinity motif (TCGATAAATT), A-tracts, AT-content, and TA dinucleotide steps, have been proposed to define H-NS binding sites (Bouffartigues et al., 2007; Gordon et al., 2011; Gulvady et al., 2018; Japaridze et al., 2017; Lang et al., 2007; OwenHughes et al., 1992; Riccardi et al., 2019; Yamada et al., 1991). However, both active transcription and other DBPs are known to affect H-NS binding patterns. Although H-NS can inhibit RNAP (Dole et al., 2004; Kotlajich et al., 2015; Lim et al., 2012; Singh et al., 2014), RNAP also can displace H-NS at some loci (Chandraprakash and Seshasayee, 2014; Rangarajan and Schnetz, 2018). Additionally, DBPs can regulate gene expression either by promoting or displacing H-NS binding, for instance in anti-silencing mechanisms (Will et al., 2015). StpA, by small Hha/YmoA-family regulators (e.g. Hha), by $\mathrm{Mg}^{2+}$, and by solutes (Dame et al., 2020). StpA forms heterodimers with H-NS whereas Hha binds to the NTD of H-NS, but not to DNA (Ali et al., 2013; Leonard et al., 2009). Both StpA and Hha stimulate bridging by H-NS (Boudreau et al., 2018; van der Valk et al., 2017; Wang et al., 2014); both also enhance silencing at subsets of H-NS regulated genes, but the extent of overlap between these subsets is unknown (Banos et al., 2009; Lucchini et al., 2009; Muller et al., 2006; Nieto et al., 2000; Srinivasan et al., 2013; Uyar et al., 2009). However, ChIP-seq results suggest that H-NS, StpA, and Hha bind to the same locations in the genome (Srinivasan et al., 2013; Ueda et al., 2013; Uyar et al., 2009); thus, their apparently differential effects on gene regulation remain to be explained. arrangement in the switch from linear to bridged conformations. DNase and $\cdot \mathrm{OH}$ footprinting experiments reveal nucleation at high-affinity sites and H-NS oligomerization with an apparent periodicity of DBD contacts every $\sim 10$ bp (Bouffartigues et al., 2007; Ulissi et al., 2014; Will et al., 2015). However, $\sim 20$ - and $\sim 30$-bp periodicities of DBD footprints also are reported. A prevalent model for H-NS filament organization based on an NTD crystal structure suggests 30-bp spacing of DBDs (Arold et al., 2010; Dame et al., 2006). Given the flexible linker connecting the DBD, a key question is whether DBDs necessarily exhibit a regular arrangement 
71 of contacts to DNA within H-NS filaments. New approaches are needed to determine how DNA

72 sequence governs DBD location and the arrangement of DBDs in an H-NS filament.

73 To gain insight into the determinants of H-NS filament formation and DBD locations in

74 filaments, we developed and applied a multimodal, multiscale approach that combines a new

75 method to reconstitute and map bacterial chromatin using HT-sequencing (Nitro-seq), a novel

76 DBD-mapping method using a tethered chemical nuclease (TEN-map), hydroxyl radical $(\cdot \mathrm{OH})$

77 footprinting, ChIP-seq, computational molecular dynamic (MD) simulations, and rigorous

78 statistical analysis and modeling. Our results reveal that DNA sequence, rather than transcription

79 or other DBPs, principally guide the pattern of gene silencing in vivo and that DBDs contact

80 DNA irregularly in an H-NS filament in patterns unaffected by bridging versus linear

81 conformations. 


\section{RESULTS}

\section{H-NS filament locations are not principally determined by transcription}

Although actively elongating RNAP can rearrange H-NS filaments near transcription-H-NS junctions (Rangarajan and Schnetz, 2018) and can more extensively alter H-NS binding genomewide when $\rho$-dependent termination is inhibited (Chandraprakash and Seshasayee, 2014), the extent to which transcription governs H-NS filament formation across the genome is unclear. To address this question, we first used ChIP-seq to determine RNAP and H-NS locations in the presence or absence of the transcription inhibitor rifampicin (Rif). Rif prevents extension of initial RNA transcripts past $3 \mathrm{nt}$, causing RNAP to relocate in vivo to promoters or promoter-like sequences (Campbell et al., 2001; Herring et al., 2005). We hypothesized that if transcribing RNAPs strongly contribute to H-NS filament patterns in cells, then trapping all RNAP at promoters with Rif would alter H-NS locations detected by ChIP-seq (Figure 1B). We treated wild-type E. coli K-12 cells with Rif and then generated ChIP-seq samples from treated and untreated cells using antibodies specific for RNAP or H-NS. Comparison of the RNAP ChIP patterns in the presence or absence of Rif revealed the expected Rif-induced redistribution of RNAP to promoters or cryptic promoters throughout the genome (Figure 1C; Figure S1-1) (Herring et al., 2005; Kim et al., 2012; Thomason et al., 2015). The majority (72\%) of identifiable RNAP peaks in Rif-treated cells were within 30 bp of a known promoter based on a genome-scale, transcription-start-site (TSS) analysis (Thomason et al., 2015), with a mean distance of $\sim 10$ bp vs. $\sim 140$ bp for a randomized control $\left(p<10^{-4}\right.$; Figure S1-2). In contrast, the H-NS binding pattern exhibited only minimal changes upon Rif treatment despite the elimination of elongating RNAPs.

To characterize the small number of filaments that did change, we used MOSAiCS to assess

105 the extent of change in filaments present at the same genomic location in untreated and Rif-

106 treated cells (Kuan et al., 2011). MOSAiCS is well-suited to map transcribed regions and H-NS

107 filaments because it can identify broad regions of enrichment relative to the input DNA prior to

108 immunoprecipitation in addition to the symmetrical Gaussian-like peaks typical for single-site

109 DNA-binding proteins. To analyze changes in H-NS filaments upon Rif treatment, we defined 
110 four classes of filaments: (1) unchanged by Rif treatment; (2) filaments with boundaries that

111 expanded (2a) or receded (2b) by more than $200 \mathrm{bp}(\sim 10 \%$ of the $\sim 2 \mathrm{~kb}$ average filament length);

112 (3) filaments that appeared only after Rif treatment; and (4) filaments that dissociated upon Rif

113 treatment. Most filaments were unchanged; class 1 included $\sim 85 \%$ of all filaments present in

114 untreated cells (266 of 301 filaments) (Figure 1D). Even when transcription was directly

115 adjacent to an H-NS filament, such as cysHIJ adjacent to the H-NS-bound cas operon, H-NS

116 filaments were not dramatically altered upon Rif treatment (Figure 1C). Class 1 unchanged

117 filaments included H-NS silenced genes with internal antisense transcription units, such as the $\beta$ -

118 glucoside utilization ( $b g l$ ) operon (Figure S1-3) (Dole et al., 2004). However, Rif treatment did

119 affect the lengths or existence of $\sim 14 \%$ of the H-NS filaments (Figure 1D), causing changes at

120 one or both ends of some filaments (class $2 ; n=30$ ), appearance of a few filaments (class $3 ; n=3$ ),

121 and disappearance of other filaments (class $4 ; n=12$ ) (Table S5).

122 We next investigated whether changes in RNAP binding to promoter or promoter-like

123 sequences explained the minimal changes in H-NS filaments upon Rif treatment. We

124 hypothesized that Rif-induced relocation of RNAP to promoters could either disrupt an existing

125 H-NS filament at sites of relocation or allow H-NS binding in previously transcribed regions.

126 Most (75\%) of the few filaments that disappeared in Rif-treated cells (class 4; $n=12$ ) overlapped

127 a Rif-induced RNAP-binding site (e.g. at the H-NS-regulated $d p s$ promoter; Figure 1D)

128 (Grainger et al., 2008) and two of the three Rif-induced filaments overlapped a location vacated

129 by RNAP (Table S5). In nearly all cases (89\%), the locations at which filaments receded (class

$1302 \mathrm{~b} ; \mathrm{n}=18$ ) corresponded to locations of Rif-induced RNAP binding (Table S5). Conversely, 55\%

131 of sites at which filaments grew (class $2 \mathrm{a} ; \mathrm{n}=12$ ) were associated Rif-induced loss of bound

132 RNAP. Together, our results indicate that active transcription elongation plays a minimal role in

133 determining locations of H-NS binding in E. coli, although the few sites at which transcription

134 does affect H-NS binding could play important roles in gene regulation.

135 H-NS nucleoprotein filaments can be reconstituted using genomic DNA in vitro

136 Even though active transcription does not principally determine H-NS filament locations, it

137 remained possible that other DBPs could play dominant roles in guiding H-NS filament 
138 formation in vivo, either by exclusion or via cooperative interactions. To test this possibility, we

139 sought to reconstitute H-NS filaments in vitro using purified proteins and chromosomal DNA to

140 compare to the H-NS binding locations in vivo. A similar approach to eukaryotic chromatin

141 reconstitution has been achieved recently and has proven informative (Krietenstein et al., 2016).

142 We predicted that any strong effects of DBPs on H-NS filament locations in vivo would be

143 evident as differences between the in vitro reconstituted filaments and in vivo ChIP-seq profiles.

144 To study H-NS filament in vitro, we reconstituted them on fragmented and purified genomic

145 DNA (gDNA), captured the reconstituted filaments by binding to nitrocellulose filters, and then

146 mapped filament locations by HT-sequencing of DNA recovered from the filters (Figure 2A;

147 Figure S2-1,2; see Methods). We call this method Nitro-seq.

148 To verify that Nitro-seq captures specific protein-DNA interactions, we compared retention 149 on filters of purified and protein-free gDNA to retention of H-NS-bound gDNA. The purified 150 gDNA gave a uniform distribution of sequence reads prior to nitrocellulose filter-binding and 151 much lower amounts of retained DNA compared to H-NS-bound samples $(\sim 1.5 \%$ of gDNA 152 retained without H-NS; Figure S2-3). Reconstitution with H-NS caused nitrocellulose retention 153 of $4-12 \%$ of the gDNA (Figure $\boldsymbol{S 2}-\mathbf{1 B}$ ), with reproducible and distinct patterns of enrichment 154 (peaks) across the genome (Figure 2B; Figure S2-4). This fraction of gDNA filter-retention by 155 H-NS was similar to the $\sim 15 \%$ of the E. coli K-12 genome bound by H-NS in vivo (Grainger et 156 al., 2006), suggesting that our Nitro-seq method captured specific H-NS binding events.

157 MOSAiCS analysis identified $407 \mathrm{H}-\mathrm{NS}$ binding regions $(\sim 1.4 \mathrm{~kb}$ on average $)$ in the Nitro-seq 158 results $($ Table 1$)$.

Importantly, the Nitro-seq H-NS-binding pattern differed from the DNA-binding pattern generated by another abundant, AT-preferring DBP in E. coli, Fis, in a control Nitro-seq

161 experiment. Fis also bound AT-rich DNA but exhibited a different pattern than H-NS consistent 162 with its distribution on chromosomal DNA in vivo (Cho et al., 2008; Kahramanoglou et al.,

163 2011); Figure S2-4; Figure S3-1A). We quantified the difference in the H-NS and Fis Nitro-seq 164 patterns by calculating the Jaccard index ( 0.2 for H-NS versus Fis; the Jaccard index measures 165 overlap of enriched regions and scales from 0-1 no overlap-complete overlap; see Methods). 
166 These results verify that Nitro-seq identifies specific patterns of protein-DNA interactions and is

167 suitable for tracking in vitro reconstitution of bacterial chromatin.

168 The in vivo pattern of $\mathrm{H}-\mathrm{NS}$ filaments is primarily determined by DNA sequence

169 To determine whether DNA sequence-specific binding by H-NS primarily explains the

170 distribution of H-NS in vivo, we compared the binding pattern of H-NS in our in vitro Nitro-seq

171 assay to in vivo ChIP-seq results (Figure 3A). Overall, the in vivo (ChiP-seq) and in vitro (Nitro-

172 seq) H-NS binding patterns were highly similar (Jaccard index $=0.6$; Figure $2 \boldsymbol{B}$, Figure $3 \boldsymbol{B}$ ).

173 However, the Nitro-seq sites fell into two classes: (1) sites that overlapped with ChIP-seq sites

174 and were similar in length $(n=297$; Jaccard index $=0.71$, covering $89 \%$ of the bp bound by H-

175 NS in vivo) and (2) sites observed only by Nitro-seq and were on average shorter than the in vivo

176 H-NS filaments $(n=112$, or $35 \%$ of the bp bound by H-NS in vitro; Figure 3B, Figure S3-2A-

177 C). Among class-1 overlapping filaments, most (255) overlapped for $\geq 90 \%$ of bp in vivo and in

178 vitro (Figure 3B, black dots). For example, the in vivo and in vitro H-NS binding patterns at the

$179 b g l$ operon were nearly identical (Figure S3-2B). We conclude that H-NS binds DNA in similar

180 patterns in vitro and in vivo, with some additional sites available in vitro.

181 We hypothesized that many of the Nitro-seq-specific filaments could be explained by

182 competition for H-NS binding in vivo by other DBPs. To test this idea, we compared the Nitro-

183 seq-specific regions to known transcription factor (TF) binding sites from RegulonDB (Santos-

184 Zavaleta et al., 2019) and to protein-occupied regions of the genome called extended protein

185 occupancy domains or EPODs (Vora et al., 2009) (Figure S3-2; ePODS can be either silenced

186 regions called $t s$ EPODS or highly expressed regions called heEPODS). If Nitro-seq-specific

187 regions are explained by binding to unmasked sites normally protected by other DBPs in vivo,

188 then they should correlate with the TF-binding sites. Alternatively if these regions are explained

189 by exclusion of H-NS by active transcription in vivo (i.e., they are normally bound by RNAP),

190 then they should correlate with heEPODS (regions of high RNAP occupancy in vivo). We found

191 that the Nitro-seq-specific filaments were enriched in RegulonDB TF-binding sites with 62 of

192112 (55\%) overlapping known TF-binding sites (Figure S3-2D). Given that TF-binding sites are

193 incompletely mapped, the observation of 55\% overlap is consistent with a majority of the class- 

compete with H-NS, such as IHF, FNR, and Fis, bind about 29 of the class-2 sites (Figure S3_ 2A; Table S6), further supporting this explanation (Stoebel et al., 2008; Will et al., 2015). In contrast, Nitro-seq-specific filaments did not overlap significantly with heEPODS, also consistent with exclusion of H-NS in vivo by DBPs and not by transcription. As expected, class-

1991 filaments were highly similar to tsEPODs $(p<0.001)$. Consistent with our H-NS ChIP results

200 (Figure 1), we conclude that the Nitro-seq-specific regions reflect sites at which H-NS is

201 excluded by other DBPs in vivo and that active transcription minimally affects H-NS filament

202 locations. Together, our results indicate that most H-NS filament locations in E. coli cells are

203 determined by DNA sequence alone with a minority of potential locations blocked by other

204 DBPs in vivo, notably by anti-silencing TFs (Will et al., 2015).

\section{H-NS and StpA exhibit indistinguishable DNA binding specificities}

Both the H-NS paralog StpA and the H-NS modulator Hha promote bridging by H-NS filaments and help silence subsets of H-NS-silenced genes with unknown overlap (Boudreau et al., 2018; Srinivasan et al., 2013; Uyar et al., 2009; van der Valk et al., 2017). Their effects on DNAsequence specificity are unknown but ChIP patterns largely indistinguishable from H-NS are reported for both, leaving their roles in silencing unexplained (Banos et al., 2009; Fukui et al.,

211 2016; Nieto et al., 2000; Uyar et al., 2009). To determine whether StpA and Hha-bound H-NS

212 filaments differ in sequence specificity from H-NS-only filaments, we applied the Nitro-seq

213 assay to StpA and Hha-H-NS filaments. Using optimized binding conditions and MOSAiCS

214 analysis (see Methods), we found that H-NS, StpA, and Hha-H-NS produced virtually

215 indistinguishable binding patterns on gDNA (Figure 2B; Figure S2-4; Figure 3; Figure S3-

216 1A; Table 1). For StpA vs. H-NS, the average densities of Nitro-seq reads were highly correlated

217 (Spearman $\rho=0.984$ ) and the MOSAiCS-predicted filaments overlapped extensively (Jaccard

218 index $=0.73$ ). StpA generated more filaments than H-NS (589 vs 407), possibly due to tighter

219 binding by StpA (Sonnenfield et al., 2001); removing the StpA-only filaments increased the

220 overlap to a Jaccard index of 0.86 (Figure 3 C). We observed a similarly high degree of overlap

221 between the H-NS and Hha-H-NS datasets (Spearman $\rho$ of average read densities = 0.993; 
222 Jaccard index of MOSAiCS-predicted filaments $=0.86$; Figure 3D; Figure S3-1A). We

223 conclude that StpA, Hha-H-NS and H-NS exhibit essentially indistinguishable sequence

224 specificities in our in vitro conditions (see Discussion).

Tethered-nuclease mapping (TEN-map) reveals H-NS DBD locations in H-NS filaments

226 Having established that in vitro reconstituted H-NS filaments recapitulate their key ability to

227 target subsets of genes in vivo, we next sought to determine the spacing, orientations, and

228 regularity of H-NS DBDs in a representative filament able to form both bridged and linear

229 configurations. To move from the genome-wide to the bp scale, we thus chose the well-

230 characterized $b g l$ operon downstream regulatory element (DRE). H-NS filaments formed on the

231 DRE are known to: (1) be determined by DNA sequence (Figure S1-3; Figure S3-2);

232 (2) switch from bridged to linear in vitro when H-NS is present above 200 monomers/kb DNA;

233 (3) be modulated by $\mathrm{Mg}^{2+}$; and (4) silence the $b g l$ operon by inhibiting both transcript elongation

234 by RNAP and, via bridging to an upstream regulatory element (URE), binding of RNAP to the

235 bgl promoter (Boudreau et al., 2018; Kotlajich et al., 2015; Nagarajavel et al., 2007; Schnetz,

236 1995) (Figure S4-1A).

237 To enable precise assignment of DBD locations in the DRE filament, we developed a

238 tethered nuclease mapping (TEN-map) assay in which an artificial chemical nuclease

$239\left(\mathrm{Fe}^{2+} \cdot \mathrm{EDTA}\right)$ is covalently tethered near but not within the DBD-DNA interface by reaction of

240 FeBABE (Fe(III) (S)-1-(p-Bromoacetamidobenzyl)ethylenediaminetetraacetate) with Cys

241 substituted for Glu-102 (Figure 4A-B). Upon activation, the tethered $\mathrm{Fe}^{2+}$.EDTA generates

242 hydroxyl radicals $(\cdot \mathrm{OH})$ that cleave the DNA backbone within $\sim 12 \AA$ of the chelated $\mathrm{Fe}^{2+}\left(\mathrm{Cheal}^{2}\right.$

243 et al., 2009). The resulting DNA cleavage pattern thus defines the location and orientation of the

244 H-NS DBD within the nucleoprotein filament. This tethered cleavage pattern differs from a

245 conventional $\cdot \mathrm{OH}$ footprinting pattern generated by $\mathrm{Fe}^{2+} \cdot$ EDTA free in solution, which

246 principally defines just solute exclusion from the DNA surface by the bound DBD.

247 To validate use of the derivatized H-NS for TEN-map, we compared it to similarly treated

248 C21S H-NS lacking the tethered nuclease and to wild-type H-NS in DNA-binding and

249 transcription experiments. Both conjugated and control H-NS bound $b g l$ DRE DNA similarly to 
wild-type H-NS in a gel-shift assay (Figure S4-2). The somewhat higher concentration requirements may reflect a known propensity of $\mathrm{H}-\mathrm{NS}$ to lose activity during biochemical manipulation (R.T. Dame, personal communication, July 2020). Of note, high concentrations of conjugated H-NS did not generate the increased mobility complex thought to reflect linear filament formation (Kotlajich et al., 2015). Conjugated H-NS slowed transcript elongation and stimulated backtrack pausing by RNAP on a $b g l$ DRE template as expected for bridged H-NS filaments. However, at $\geq 200$ monomer/kb both conjugated H-NS and control C21S H-NS treated with FeBABE (i.e., treated but unconjugated H-NS) continued to inhibit RNAP elongation indicative of constitutive H-NS bridging (Figure S4-3D), whereas at $\geq 200$ monomer/kb wildtype H-NS and untreated C21S or C21S-E102C H-NS allow faster RNAP elongation indicative of switching to linear H-NS filaments (Figure $\boldsymbol{S 4 - 3 A - C )}$ (Boudreau et al., 2018; Kotlajich et al., 2015). This observation suggests that the conjugated H-NS may be unable to form linear filaments, either due to partial inactivation by FeBABE treatment making it impossible to reach $\geq 200$ active monomer/kb or due to constitutive bridging by conjugated H-NS as observed for StpA (Boudreau et al., 2018; see Discussion). We concluded that conjugated H-NS was suitable for TEN-map analysis of DBD positions in bridged but not linear $b g l$ DRE filaments.

To probe the locations of H-NS DBDs on DNA by TEN-map, we used a 146-bp $b g l$ DRE fragment that could accommodate 10-15 H-NS monomers (assuming an H-NS binding site size of 10-15 bp; (Amit et al., 2003; Ulissi et al., 2014). The 146-bp fragment enabled single-nt resolution by denaturing PAGE of multiple DBDs on either the template $(\mathrm{T})$ or non-template (NT) strand, depending on the location of a ${ }^{32} \mathrm{P}$-end-label (Figure $4 \boldsymbol{C}$, Figure $S 4-1 B$ ). After formation of filaments, phosphodiester backbone cleavage via hydrogen abstraction from DNA sugars was triggered by addition of $\mathrm{H}_{2} \mathrm{O}_{2}$ and ascorbic acid to generate $\cdot \mathrm{OH}$ by the Fenton reaction (Balasubramanian et al., 1998). We observed robust cleavage patterns on both the $\mathrm{T}$ and NT strands indicated DBD locations on the $b g l$ DRE fragment (Figure 4D, Figure S4-4A). Cleavage was dependent on the presence of ascorbic acid, $\mathrm{H}_{2} \mathrm{O}_{2}$, and Fe EDTA tethered to $\mathrm{H}$ NS, indicating that it resulted from $\mathrm{H}-\mathrm{NS}$ binding and not from $\cdot \mathrm{OH}$ formed by untethered Fe·EDTA (Figure 4D, top panel). 
Peaks of cleavage were spaced irregularly at intervals of 6-21 nt on the T strand and 9-14 nt on the NT strand (Figure $4 \boldsymbol{E}$ and $\boldsymbol{F}$; NT, red arrows; T, blue arrows) and remained unchanged in 280 location up to $5 \mu \mathrm{M}$ Fe·EDTA-H-NS. We quantified the H-NS concentration-dependence of 281 cleavage using linear approximations of the change in normalized peak areas (Figure S4-4 and 282 5). Large, positive slopes indicating robust H-NS-dependent cleavage were spaced $\sim 10-15$ bp. 283 This spacing is consistent with previous H-NS footprint analyses using untethered probes on different DNA fragments in which regions of protected DNA occurred with 10-20 bp periodicity (Bouffartigues et al., 2007; Dame et al., 2006; Ulissi et al., 2014; Will et al., 2018).

To verify that TEN-map patterns reflected tethered nuclease cleavage, we compared them to untethered ' OH cleavage of DNA alone or of DNA bound to wild-type H-NS (Figure S4-6). As expected if conjugated and unconjugated H-NS DBDs protect the same sites, the TEN-map patterns had features in common with untethered $\cdot \mathrm{OH} H-\mathrm{NS}$ footprints, reflecting DNA protected by bound DBD, but were clearly different, reflecting localized TEN-map cleavages.

291 Both tethered and untethered $\cdot \mathrm{OH}$ cleavage were also easily distinguishable from background -OH cleavage of DNA alone. These results confirmed that TEN-map yields valuable information that can be used to locate DNA contacts of the H-NS DBDs and that $\cdot \mathrm{OH}$ footprints provide an independent source of information to map DBD-DNA contacts.

To test whether H-NS binding patterns are affected by flanking sequence or DNA end positions, we first compared cleavage of the 146-bp bgl-DRE fragment to that of a 151-bp 297 fragment with identical internal sequence and 5 bp added to the downstream end (Figure S4298 7A). The identical cleavage patterns of these fragments established that the DBD locations 299 reported by TEN-map depended on the underlying sequence rather than being phased from a 300 nearby DNA end (Figure S4-7B), as expected from prior results (Bouffartigues et al., 2007). To 301 test whether the patterns seen on a short bgl-DRE change in longer filaments, we compared 302 results on the 146-bp fragment to TEN-map analysis of a 549-bp bgl DRE-containing fragment 303 that was extended toward $\mathrm{P}_{b g l}$ but retained the same downstream ${ }^{32} \mathrm{P}$-labeled end (Figure S4$304 \quad 7 C$ ). The tethered-cleavage patterns were nearly identical for both DNA fragments (Figure S4305 7D), except for a new peak at position $\sim 645$ on the longer fragment that likely resulted from 
stabilization of the upstream H-NS filament. We conclude TEN-map reports DBD locations based only on the underlying DNA sequence and that these locations on the $b g l$ DRE are spaced irregularly at intervals of roughly $5-20 \mathrm{bp}$.

\section{H-NS DBD locations are unchanged in obligately bridged filaments or by $\mathbf{M g}^{2+}$}

310 We next sought to confirm that TEN-map reported H-NS DBD locations in bridged filaments by

311 forming obligately bridged filaments using a previously developed bridging pull-down assay

312 (van der Valk et al., 2017) (Figure 5A; Figure S5-1A). In this assay, H-NS-DNA filaments are

313 captured from solution by DNA-coated magnetic beads. Capture requires H-NS bridging

314 between the bead-bound and unbound DNAs. We incubated streptavidin beads pre-loaded with a 315 685-bp, biotinated bait DNA with a mixture of Fe·EDTA-H-NS, unmodified H-NS, and the 549-

316 bp $b g l$ DRE-containing fragment (Figure 5A; the $b g l$ DRE fragment lacked biotin and was 5'-

$317 \quad{ }^{32}$ P-end-labeled on the T strand). Bridged filaments, whose formation was unaffected by the

318 presence of conjugated H-NS, were magnetically separated from unattached filaments and TEN-

319 map cleavage was activated. These obligately bridged cleavage patterns were indistinguishable

320 from those observed without bead-based capture (compare Figure S4-7D to Figure 5A; Figure

321 S5-2), confirming that the TEN-map assay reports the locations of bridged H-NS DBDs. As an

322 additional test, we asked how elevated $\mathrm{Mg}^{2+}$, which stimulates bridging at concentrations $>5 \mathrm{mM}$

323 (Liu et al., 2010), would affect the TEN-map assigned DBD locations. We found that DBD

324 locations were unaffected by elevated $\mathrm{Mg}^{2+}(8 \mathrm{mM}$ vs. $\sim 0.3 \mu \mathrm{M})$ (Figure 5B, Figure S5-3). Our

325 results are consistent with constitutive bridging by conjugated H-NS in the TEN-map assay and

326 also are consistent with a previous conclusion that changing $\mathrm{Mg}^{2+}$ concentration does not alter

327 DBD locations (Will et al., 2018).

\section{DBD · OH footprints on DNA are unchanged during bridged-to-linear switching}

329 Although TEN-map reveals the location of DBDs in bridged filaments, we wanted to compare

330 these locations on bridged and linear filaments. To enable this comparison, we examined

331 untethered $\mathrm{OH}$ footprints of wild-type H-NS on the $b g l$ DRE encompassing previously validated

332 bridged-to-linear switching by increasing H-NS/DNA ratios (Kotlajich et al., 2015) (Figure 6A).

333 We normalized $\cdot \mathrm{OH}$ cleavage as a function of $\mathrm{H}-\mathrm{NS}$ concentration to the unprotected cleavage 
334 pattern to correct for intrinsic DNA reactivity (Burkhoff and Tullis, 1988) (Figure 6B-D, Figure

335 S6-1). Strikingly, the H-NS footprint patterns did not change as H-NS concentration was

336 increased from a range that favors bridging to well above the concentration known to switch

337 filaments to the linear conformation. This unchanging $\cdot \mathrm{OH}$ footprint pattern indicates that $\mathrm{H}-\mathrm{NS}$

338 DBD locations on DNA are the same in bridged and linear filaments. The unchanging pattern of

339 H-NS also enabled us to analyze protection of DNA from $\cdot \mathrm{OH}$ as a function of $\mathrm{H}-\mathrm{NS}$

340 concentration by calculating linear slopes and thus assign a magnitude of protection to each

341 position in the $b g l$ DRE (Figure S6-1D,F; Figure S6-2). This analysis revealed that sites of

342 maximal DBD protection were irregularly spaced at intervals of 5-24 nt in both bridged and

343 linear filaments, similar to the spacing observed in bridged filaments by TEN-map (see bars at

344 the bottoms of graphs, Figure 6 C-D). These results can be explained if H-NS DBDs occupy the

345 same irregularly spaced locations on DNA in bridged and linear H-NS nucleoprotein filaments.

346 MD simulation of TEN-map and independent footprint cleavage patterns allows DBD

347 positioning

348 To determine locations and orientations of the H-NS DBD on the $b g l$ DRE in molecular terms,

349 we needed a way to predict the expected TEN-map and footprint cleavage patterns for a given

350 DBD location and orientation. To generate these predictions, we used molecular dynamics (MD)

351 simulations. For TEN-map analysis, we first predicted the position of the conjugated Fe·EDTA

352 - and thus the position of the catalytic $\mathrm{Fe}^{2+}$ — with respect to DNA and calculated solvent

353 accessible areas (Figure 7). The simulations generated a probability distribution of distances

354 between the $\mathrm{Fe}^{2+}$ and each nt in the two duplex DNA strands to which H-NS is bound (the two

355 strands were arbitrarily designated A and B for modeling). We assumed that the cleavage

356 propensity at each $n t$ is directly proportional to the local concentration of $\cdot \mathrm{OH}$ at that nt, which

357 we estimated by a diffusion model for $\cdot \mathrm{OH}$ centered at the $\mathrm{Fe}^{2+}$ (Cheal et al., 2009). Because

358 some nucleotides are shielded from the solvent by the bound DBD, we scaled the concentration

359 of radicals according to the solvent-accessible surface area of the putative cleavage site (Figure

360 S7-2) (Balasubramanian et al., 1998). Modeling based on these assumptions yielded an estimate

361 of the relative cleavage propensity at each nt in the duplex DNA bound by the DBD (Figure 7A). 
362 When displayed on B-form DNA, foci of cleavage were evident on each DNA strand offset from

363 the location of the DBD in the minor groove (Figure $7 \boldsymbol{B}$ ). The maximum peak of cleavage was

364 predicted to occur at $\mathrm{nt}+10$ of strand $\mathrm{A}$ and was flanked by two smaller predicted cleavage peaks

365 in strand B. Adjacent to these sites of high predicted cleavage were sites of particularly low

366 predicted cleavage, which resulted from shielding by the bound DBD.

To identify specific DBD positions in the $b g l$-DRE filament, we developed a statistical method to compare the experimentally measured TEN-map pattern (Figure $\boldsymbol{S 4 - 5 A}$ ) to the MDpredicted cleavage pattern for each possible DBD position and orientation (Figure 7B). For each comparison, the Manhattan distance between the simulated pattern and the experimentally-

371 determined cleavage pattern was calculated; the Manhattan distance is the sum of absolute

372 differences in all dimensions between of sets of points with a lower score indicating a closer

373 match (see Methods). This analysis predicted seven unique binding sites with match scores lower 374 than $95 \%$ of all match scores from 1000 different sets of shuffled data, which we interpreted to 375 be statistically significant (Figure 7 C). Given the noise in both the predictions and experimental 376 data, we interpreted predicted binding sites separated by $\leq 3$ bp as one binding site (e.g. n663F 377 and $n 664 \mathrm{R}$ were assigned as one binding site; $\mathrm{n}$ indicates assignment is from tethered nuclease 378 data; F or R indicate forward or reverse orientation of the DBD relative to the coding direction of 379 the $b g l$ operon; Figure $7 \boldsymbol{B}$ ). To visualize this arrangement of DBDs, we placed the center of the 380 DBD (black triangle in Figure 7 B) on B-form DNA at the locations with a statistically 381 significant match score in the appropriate orientation (Figure S7-1). This model of the H-NS 382 DBDs confirmed that occupation of these seven binding sites on one duplex DNA is sterically 383 feasible. Overall, our TEN-map analysis, which applies to bridged H-NS filaments, predicted 384 four DBDs bound in the forward orientation $(\mathrm{n} 642 \mathrm{~F}, \mathrm{n} 676 \mathrm{~F}$, n695F, and $\mathrm{n} 711 \mathrm{~F})$, two bound in 385 the reverse orientation (n685R and n706R), and one (n663F/n664R) bound either in alternating 386 orientations or in an indeterminate orientation. An alternating orientation could result from the 387 DBD changing orientation dynamically or being bound in opposite orientations on different 388 DNA molecules; noise in our experimental data and MD predictions precludes definitive 389 assignment of the n663F/n664R DBD. 
As an independent way to predict DBD locations for both linear and bridged filaments, we

391 applied a statistical method similar to that used for TEN-map to the untethered $\cdot \mathrm{OH}$ footprints of

392 wild-type H-NS on the $b g l$ DRE using calculated Manhattan distances for solvent accessibility-

393 based predictions (Figure S6-2; Figure S7-2A-B; see Methods). Because the solvent

394 accessibility pattern was asymmetrical, we matched the footprint data to predictions for both

395 DBD orientations. This analysis predicted four unique binding sites, which we modeled onto B-

396 form DNA (Figure $\boldsymbol{S}$ 7-2C). Two footprint-predicted DBDs were in the forward orientation

397 (p694F and p711F) and two were either bound in alternating orientations or bound in

398 indeterminate orientation (p665F/p668R and p685F/p688R).

The footprint-based and TEN-map predictions of DBD positions on the $b g l$ DRE coincided,

401 Three features of this DBD arrangement stood out. First, we observed variation in the intervals

402 between the location of DBDs on DNA (5-20 bp). Second, both forward and reverse DBD

403 orientations were observed. Third, some DBDs (e.g. at 663-669) exhibited either indeterminate

404 or alternating orientations. Together, these results reveal that DBDs in both bridged and linear H-

405 NS filaments do not contact DNA with rigidly fixed spacing and orientation but rather assume positions and orientations within a window of possible locations based on the highest affinity to

407 local DNA sequence features (see Discussion).

408 H-NS DBD positioning is associated with sequence-determined DNA structure

409 Having established from independent $\cdot \mathrm{OH}$ footprint and TEN-map analyses that DBD location

410 and orientation appears to be set by features of DNA sequence within both bridged and linear

411 filaments and that DNA sequence is the principal determinant of the genome-scale distribution of

412 H-NS, we next sought to identify sequence-based parameters that best explain the H-NS

413 distribution genome-wide (i.e., we sought to use bp-scale information to predict genome-scale H-

414 NS locations). Previous work suggests that H-NS DBDs exhibit variable preference for AT-rich

415 4mer sequences (AAAA, AAAT, AATT, ATTT, TTTT, ATAA, ATAT, ATTA, AATA, TAAA,

416 TAAT, TATA, TATT, TTAA, TTAT, or TTTA) with TA-step-containing 4mers being most

417 favored, as well as having increased affinity when the 4mers are located in regions of high A/T 
418 content (Gordon et al., 2011). These sequence motifs (meaning a sequence pattern, not a position

419 weight matrix, PWM) were postulated to favor DBD binding because they form a narrow minor

420 groove width (MGW), which H-NS has been shown to bind (Gordon et al., 2011). In addition to

421 sequence motifs per se, it also is possible to predict DNA structural features on a genome-scale

422 such as MGW, propeller twist (ProT), and electrostatic potential in the minor groove (EP) (Chiu

423 et al., 2017; Chiu et al., 2016; Zhou et al., 2013).

424 To determine which sequence motifs and sequence-defined structural features best predict

425 H-NS binding, we first asked which best explained the H-NS DBD arrangement in the $b g l$ DRE

426 (Figure 8A). We found that our high-confidence DBD locations corresponded most closely to

427 TA-step and A-tract sequences with a narrow MGW, negative propeller twist, and highly

428 negative electrostatic potential. Using this set of sequence motifs and structural features that best

429 explained DBD locations in the $b g l$ DRE as a guide, we next sought to predict the genome-wide

430 distribution of H-NS filaments. We performed this analysis by comparing the H-NS bound status

431 of $1000 \mathrm{bp}$ windows staggered every $250 \mathrm{bp}$ across the $E$. coli genome to the sequence and

432 structural features associated with those windows. We used least absolute shrinkage and

433 selection (LASSO) logistic regression trained on H-NS ChIP-seq data to predict the binding

434 pattern of H-NS, StpA, and Hha-H-NS (see Methods). LASSO regression yielded a minimal set

435 of DNA features that best explained the distribution of H-NS (Figure $8 \boldsymbol{B}$ ). We found that a

436 simple model with only TA-step abundance, A/T content, and A-tract abundance was able to

437 predict H-NS ChIP-seq regions with high precision and recall (Figure 8C). Strikingly, these

438 sequence features, which also best matched DBD locations in an H-NS filament (Figure 8A), did

439 not gain significant predictive power when structural features were incorporated into the model.

440 Thus, both DBD locations and H-NS genomic distribution are best predicted by the combination

441 of three sequence features among which TA-step abundance is the most informative.

442 To test this model trained on H-NS ChIP-seq data, we next asked if it also predicted

443 genome-scale H-NS binding locations in the presence of Rif and in the in vitro Nitro-seq

444 experiments using chromatin reconstituted with H-NS, with StpA, or with H-NS:Hha. We found

445 this same sequence-motif-only model worked well to explain genome-scale binding distributions 
446 in these different experiments (Figure 8C). As expected, the same model did a poor job of

447 predicting Fis binding sites in vitro, confirming its discriminatory power to differentiate H-NS

448 peaks from Fis peaks despite the similarity in A/T preference of these two proteins. Validating

449 our prediction approach overall, however, a similar model trained instead on the Fis Nitro-seq

450 data identified the RegulonDB Fis PWM as the most important feature for predicting the Fis

451 Nitro-seq distribution (Figure S8-1A). Although it is notable that LASSO selected only DNA

452 sequence motifs and not structural features for the final H-NS model, it is possible that the

453 relevant structural information is fully captured by the sequence motifs. Indeed, models trained

454 on H-NS Nitro-seq rather than ChIP-seq data selected the same three variables and performed

455 with similar high precision and recall (Figure S8-1B). We conclude that genome-scale H-NS,

456 StpA, and H-NS:Hha distributions can be predicted by DNA sequence motifs related to those

457 that explain DBD locations on the bp scale. 


\section{DISCUSSION}

460 The biological function of H-NS depends on its complex structural properties, which includes

461 alternating head-head, tail-tail oligomerization of its NTD, flexibly connected DBDs,

462 paralogous subunits (e.g. StpA), and small-protein modulators (e.g. Hha) that combine to form

463 as-yet unresolved filament structures with DNA. This complexity enables formation of multiple

464 filament types, notably $(i)$ bridged nucleoprotein filaments that inhibit both transcription

465 initiation and elongation and (ii) linear filaments that principally inhibit only initiation. Our

466 multimodal-multiscale approach, which combined new genome-scale reconstitution and

467 sequencing methods (Nitro-seq), conventional footprinting, direct detection of DBD locations

468 (TEN-map), molecular dynamics, and bioinformatics yielded three principal insights: (i) the

469 genome-wide locations of H-NS binding in vivo are determined principally by DNA sequence-

470 binding preferences, which are unchanged by the paralog StpA or modulator Hha; (ii) the DBDs

471 in an H-NS filament do not contact DNA with precisely fixed spacing but rather adjust both

472 contact point and orientation along adjacent minor groove positions; and (iii) HNS DBD

473 locations do not change in the switch from linear to bridged filaments, supporting a model of

474 DBD hemi-sequestration in linear filaments. We discuss each insight in the context of an overall

475 model of H-NS binding in which DNA sequence dictates both the location of H-NS filaments

476 genome-wide and the arrangement of DBDs within the filaments (Figure 8).

\section{H-NS genomic distribution is primarily determined by DNA sequence}

478 Multiple contributions to H-NS genomic distribution have been described, including AT-rich-

479 sequence-specific binding (Grainger et al., 2006; Kahramanoglou et al., 2011; Lang et al., 2007;

480 Lucchini et al., 2006; Navarre et al., 2006), competition with transcription (Chandraprakash and

481 Seshasayee, 2014; Rangarajan and Schnetz, 2018), and competition with other DBPs (e.g. in

482 anti-silencing gene regulation) (Will et al., 2015). However, the extent to which each of these

483 factors contributes to H-NS genomic distribution is currently uncertain. We predicted that if

484 transcription or DBPs played a dominant role in determining genome-scale H-NS filament

485 patterns, then in vitro reconstitution of H-NS filaments on genomic DNA would yield different

486 H-NS binding patterns than observed in vivo by ChIP-seq. In fact, we found only minor 
differences between in vitro and in vivo H-NS binding patterns. Differences were attributable to limited instances of transcription competing with H-NS binding and primarily to DBPs that displace H-NS in vivo such as anti-silencing TFs (Figures 1-3). Further confirming the dominant role of DNA sequence in determining H-NS locations in vivo, a statistical model based on only three DNA sequence features (A/T richness, TA steps, and A tracts) could robustly predict H-NS

492 binding genome-wide (Figure 8). These findings do not contradict the reported roles of active transcription or of anti-silencing by TFs in H-NS binding (Rangarajan and Schnetz, 2018; Will et al., 2015). Rather, our findings suggest that active transcription and DPBs affect H-NS distribution in limited ways at discrete sites with important regulatory consequences (e.g. TFrearrangement of $\mathrm{H}-\mathrm{NS}$ at promoters during anti-silencing) but that the overall genomic distribution of H-NS is determined by DNA sequence rather than by other proteins bound to DNA (Figure 8E).

Our findings that StpA and Hha do not affect the sequence-specificity of H-NS filament

500 formation have important implications for how StpA and Hha confer greater regulation on 501 subsets of genes bound by H-NS (Banos et al., 2009; Lucchini et al., 2009; Muller et al, 2006).

502 Nitro-seq established that StpA and Hha-H-NS bind the same subset of DNA sequences as 503 bound by H-NS-only filaments (Figure 2B; Figure S2-1; Figure 3; Figure S3-1A; Table 1).

504 These results agree with previous ChIP results showing extensive overlap between H-NS, StpA, 505 and Hha throughout the genome (Ueda et al., 2013; Uyar et al., 2009), but raise an obvious 506 question. If StpA and Hha do not alter the sequence-specificity of H-NS binding, then how do 507 they accomplish preferential regulation of subsets of H-NS bound genes? 
515 at higher temperatures where H-NS affinity may be reduced (Bouffartigues et al., 2007; Shahul

516 Hameed et al., 2019). A second possibility is that the differential effects on gene expression

517 could reflect differential ability of certain sequences to promote bridging (see below), since both

518 StpA and Hha confer constitutive bridging on H-NS filaments (Boudreau et al., 2018; van der

519 Valk et al., 2017), and thus could have greater effects on sequences that favor linear H-NS-only

520 filament formation.

521 Our characterization of H-NS sequence preferences may also aid prediction of H-NS

522 binding locations in other bacteria. Previous attempts at predicting H-NS binding locations relied

523 on similarity to the high-affinity consensus sequence TCGATAAATT (Lang et al., 2007). We

524 found sequence characteristics of A/T richness, TA steps, and A tracts were sufficient to explain

525 the genome-scale distribution of filaments in vivo and of H-NS-only, H-NS-StpA, and H-NS-

526 Hha filaments in vitro (Figure 8B). The PWM based on the high-affinity consensus sequence as

527 well as DNA structure parameters added negligible predictive value, making this prediction

528 relatively simple to apply. Application of the algorithm to other enterobacterial species that rely

529 on H-NS will predict binding locations that can be validated by H-NS ChIP-seq.

\section{DBD position and orientation within H-NS filaments is flexible and set by DNA sequence}

531 Our finding that both H-NS DBD position and orientation are flexible within H-NS filaments is

532 consistent with reported binding patterns of H-NS on DNA (Badaut et al., 2002; Bouffartigues et

533 al., 2007; Tupper et al., 1994; Ulissi et al., 2014; Will et al., 2018). These previous footprinting

534 experiments typically revealed semi-regular spacing of $\sim 10 \mathrm{bp}$ between H-NS protected sites, but

535 also variation in spacing over a 5-30 bp range between a subset of sites. When bridged H-NS

536 filaments are pulled apart by $\sim 22 \mathrm{pN}$ force on beads attached to the two bridged DNAs, H-NS

537 contacts are lost in steps of multiples of $\sim 11$ bp ( $\sim 1$ helical turn of B-form DNA) (Dame et al.,

538 2006). However, many 11-bp steps give no force signature yielding a mean step size closer to

539 two helical repeats. Possibly some contacts are too weak to give a force signature; alternatively,

540 some DBDs could remain bound to the NTD yielding an $\sim 22$ bp spacing. Further, deviations in

541 apparent DBD-DNA contact positions by $\pm \sim 2$ bp are frequent. In agreement with this single-

542 molecule view of variation in contacts, we observed a correlation between the exact DBD 
543 contact positions and the DNA sequence that suggests DNA sequence may dictate a lowest free

544 energy arrangement of DBDs within the constraints imposed by their flexible tethers to the H-NS

545 oligomer. Specifically, DBDs were located on the $b g l$ DRE fragment at sequence and shape

546 features that confer high-affinity for H-NS (e.g. TA steps, A-tracts leading to narrow minor

547 groove width and negative EP), but were not observed at lower-affinity sites lacking these

548 features (Gordon et al., 2011) (Figure 8A). Thus, the precise position and orientation DBDs on

549 the minor groove as it aligns with the H-NS filament in every helical repeat is likely dictated by

550 the best minor groove complement in shape and charge to the DBD within reach of the linker.

551 The larger spacings of two or more helical repeats in contacts may reflect the absence of suitable

552 binding features near a given tail-tail junction in the H-NS oligomer so both DBDs at a given

553 junction remain associated with the NTD. Of note, we cannot exclude the possibility that both

554 DBDs at a junction contact one DNA duplex adjacent to each other, which could explain the

555 706R and 711F DBDs in our bgl DRE model (Figure 7D).

What can this flexibility in DBD arrangement tell us about the structure of H-NS filaments?

Our results do not define a specific conformation for the H-NS head-head, tail-tail oligomer.

However, the DBD locations we find are inconsistent with the oligomer structure observed in an

H-NS crystal that places the tail-tail junction to which the DBDs are tethered at $\sim 30$ bp intervals relative to B-form DNA (Arold et al., 2010). If DBDs contact B-form DNA every 10.5 bp in a

561 bridged filament in which one of the two DBDs at each junction binds one DNA duplex and the

562 other DBD binds the other duplex, then the H-NS NTD must adopt a conformation in which the

563 tail-tail junctions are $\sim 10.5$ bp or $33 \AA$ apart. The crystal structure of the NTD oligomer places

564 these junctions $\sim 95 \AA$ apart, nearly three times the 10.5-bp distance on B-form DNA. Although

565 H-NS binding may distort DNA, it is unlikely to increase the helical pitch by a factor of 3.

566 Consistent with a shorter distance between tail-tail junctions, other conformations of the NTD

567 have been observed by MD simulation (Shahul Hameed et al., 2019; van der Valk et al., 2017);

568 these alternatives could enable explain the variable but on average $\sim 11$ bp spacing we and others

569 observe between neighboring DBDs. Future studies, possibly by cryo-EM, of a complete H-NS-

570 DNA filament are needed to resolve how H-NS monomers assemble to enable the semi-regular

571 but flexible orientations we observed for the H-NS DBDs. 
572 H-NS DBD contacts to DNA do not change in the switch from bridged to linear

573 conformations

574 One of the most intriguing features of H-NS-based gene regulation is the capacity of H-NS

575 filaments to switch from binding one DNA duplex as a linear filament to binding two DNA

576 duplexes in a bridged conformation. The extent of DNA bridging in vivo and whether it varies

577 among genes or conditions remains unknown. However, the fact that H-NS can block transcript

578 elongation only in the bridged conformation where it creates topological traps to elongating

579 RNAP (Kotlajich et al., 2015; Boudreau et al., 2018) and the clear evidence that H-NS modulates

580 transcription elongation in vivo by stimulating $\rho$-dependent termination (Chandraprakash and

581 Seshasayee, 2014; Peters et al., 2012; Saxena and Gowrishankar, 2011) provides strong evidence

582 that bridging occurs to some extent in vivo. A structural basis for switching of H-NS between

583 linear and bridged filaments remains to be established.

The Dame and Arold groups have proposed a hemi-sequestration model of H-NS DBD location that can explain linear-to-bridged switching (van der Valk et al., 2017; Shahul Hameed et al., 2018). In the hemi-sequestration model, one of the two DBDs present at each tail-tail dimerization interface in an H-NS filament binds to DNA and the second DBD is sequestered through interactions with the N-terminal dimerization domain, possibly aided by buckling of the $\alpha$-helix connecting the two dimerization interfaces. Thus, the hemi-sequestered or closed conformation enables only linear filament formation whereas the open conformation, which is

591 favored by binding of $\mathrm{Mg}^{2+}$ or Hha and possibly constitutive in StpA (Boudreau et al., 2018), 592 releases the sequestered set of DBDs so they can bridge to a second DNA duplex (Figure 1A; 593 Figure 8D).

594 Our results validate the hemi-sequestration model because we observe no changes in the 595 locations of DBDs on the DNA between filaments that are obligately bridged (Figure 5A) and 596 filaments formed in conditions known to favor linear filament formation (high wild-type H597 NS/DNA ratio Figure 6) (Kotlajich et al., 2015). Even though the precise $b g l$-DRE contacts and 598 orientation of the DBDs appear to be set by local sequence features and are sometimes weak or 599 absent at the typical $\sim 10.5$ bp intervals, these patterns show no evident change in conditions 
600 favoring linear conformations where twice as many DBDs could be available for DNA contacts

601 (Figure 6). This same observation of no change in footprints at different H-NS/DNA ratios or at

602 high versus low $\mathrm{Mg}^{2+}$ concentration thought to govern linear-bridged preference has been

603 reported by several other groups (Badaut et al., 2002; Bouffartigues et al., 2007; Tupper et al.,

604 1994; Ulissi et al., 2014; Will et al., 2018). Taken together, these data strongly favor the hemi-

605 sequestration model of H-NS linear-to-bridged filament switching.

\section{Conclusion}

607 Our multimodal-multiscale approach to understanding the genome-scale basis of H-NS filament

608 formation and the rules governing H-NS DBD contact to DNA has clarified how linear and

609 bridged H-NS filaments are organized on DNA. The challenges now are to translate this

610 understanding to molecular resolution structures of these filament conformations and to develop

611 approaches that can distinguish locations of linear versus bridged filaments in living cells.

612 


\section{MATERIALS AND METHODS}

\section{Materials}

615 Reagents were obtained from ThermoFisher (Waltham, MA, USA) or Sigma-Aldrich (St. Louis,

616 MO, USA), unless otherwise specified. Oligonucleotides ( Table S2) were obtained from IDT

617 (Coralville, IA); ribonucleotide triphosphates (rNTPs), from Promega (Madison, WI); [ $\left.{ }^{-32} \mathrm{P}\right] \mathrm{GTP}$

618 and $\left[\gamma^{-32} \mathrm{P}\right] \mathrm{ATP}$, from Perkin Elmer (Waltham, MA); polyethyleneimine (PEI, avg. MW =

619 60,000), from Acros Organics;

620 (S)-1-(p-Bromoacetamidobenzyl)ethylenediaminetetraacetate (BABE), from Santa Cruz

621 Biotechnology; and DNA-modifying enzymes, from New England Biolabs (NEB; Ipswich, MA).

\section{Plasmid preparation}

623 An expression plasmid for wild type H-NS (pWTHNS) was constructed by Gibson assembly

624 using NEB HiFi reagents (Table S1). The hns coding sequence was amplified from MG1655

625 genomic DNA using primers \#11779 and \#11780 (Table S2) and the pET21d backbone was

626 amplified from pHNS using primers \#11781 and \#11782 before assembly. To introduce point

627 mutations in pWTHNS or pHisHNS (Table S1), single primer site-directed mutagenesis was

628 performed with Q5 DNA polymerase (NEB) and primer \#8964 to change the Cys21 codon

629 (TGT) to a Ser (AGT) in pHNSC21S. To create pHNSC21SE102C, primer \#8964 was used to

630 mutate the Cys21 codon and \#8963 was used to change the Gln102 codon (GAA) to Cys (TGT).

631 Sequences of all plasmids described here were confirmed in key regions by Sanger sequencing.

\section{Protein Purification}

633 Native StpA and Hha were purified as described previously (Boudreau et al., 2018). C-terminally

634 His6x-tagged H-NS was purified as described previously (Kotlajich et al., 2015).

635 H-NS C21S purification

636 H-NS C21S was purified as described for non-tagged H-NS in (Boudreau et al., 2018) with the

637 following modifications. Briefly, BL21 $\lambda$ DE3 cells transformed with pHNSC21S and pHiC were

638 grown in $2 \mathrm{~L}$ of LB supplemented with ampicillin $(100 \mu \mathrm{g} / \mathrm{mL})+$ gentamycin $(10 \mu \mathrm{g} / \mathrm{mL})$ to

639 OD600 $\sim 0.6$ before inducing expression of H-NS C21S with $0.5 \mathrm{mM}$ isopropyl $\beta$-D-1- 
640 thiogalactopyranoside (IPTG) for $2 \mathrm{~h}$ at $37^{\circ} \mathrm{C}$. Pelleted cells were lysed by sonication. H-NS

641 C21S was precipitated with addition of $0.6 \%(\mathrm{w} / \mathrm{v})$ PEI and eluted with PEI elution buffer

642 containing $0.8 \mathrm{M} \mathrm{NaCl}$ followed by ammonium sulfate precipitation. Precipitated H-NS was

643 dissolved in nickel binding buffer and applied to a HisTrap $\mathrm{Ni}^{2+}$ column (GE Healthcare).

644 Fractions containing H-NS were mixed with TEV protease to remove the N-terminus His ${ }_{6 x}$-tag.

645 H-NS C21S was reapplied to the nickel column. Non-tagged protein was collected in the flow

646 through and dialyzed against Heparin Buffer $(20 \mathrm{mM}$ Tris- $\mathrm{HCl}$ pH 7.5, $130 \mathrm{mM} \mathrm{NaCl}, 100 \mu \mathrm{M}$

647 EDTA, 5\% glycerol, $1 \mathrm{mM}$ dithiothreitol (DTT)). H-NS C21S was then applied to a HiTrap Hep

648 column (GE Healthcare) and eluted with Heparin Buffer containing $1 \mathrm{M} \mathrm{NaCl}$. Fractions

649 containing H-NS C21S were pooled and dialyzed against Binding Buffer $(20 \mathrm{mM}$ Tris-HCl pH

$6507.2,10 \%$ glycerol, $1 \mathrm{mM}$ DTT) supplemented with $130 \mathrm{mM} \mathrm{NaCl}$. To concentrate H-NS, the

651 protein was applied to a Resource Q column (GE Healthcare). The column was washed with a

652 130-200 mM NaCl gradient in Binding Buffer over 7 column volumes. H-NS C21S was eluted

653 in $3 \mathrm{~mL}$ Binding Buffer with $500 \mathrm{mM} \mathrm{NaCl}$. Fractions containing H-NS were dialyzed against

654 H-NS Storage Buffer (20 mM Tris-HCl pH 7.5, $0.3 \mathrm{M}$ potassium acetate, 10\% glycerol) before

655 storing at $-80{ }^{\circ} \mathrm{C}$. Protein purity $(\sim 90 \%)$ and yield $(\sim 5 \mathrm{mg})$ were determined by SDS-PAGE and

656 Qubit, respectively.

657 Native WT and C21S E102C H-NS purification

658 BL21 2 DE3 cells were transformed with pWTHNS or pHNSC21SE102C before deleting hns by

$659 \mathrm{P} 1$ transduction using a lysate from the $\Delta h n s:$ :kan Keio strain (Coli Genetic Stock Center \#9111;

660 (Baba et al., 2006). After confirming the hns deletion by PCR, 2 L of cells were grown

661 aerobically in Luria Broth- $0.5 \% \mathrm{NaCl}$ supplemented with $100 \mu \mathrm{g}$ ampicillin $/ \mathrm{mL}$ at $37^{\circ} \mathrm{C}$ to

662 apparent $\mathrm{OD}_{600} 0.5$ before inducing H-NS expression by addition of IPTG to $1 \mathrm{mM}$ and

663 carbenicillin to $250 \mu \mathrm{g} / \mathrm{mL}$ followed by shaking at $16 \mathrm{~h}$ at $25^{\circ} \mathrm{C}$. Cells were pelleted at $3,000 \times g$

664 for $15 \mathrm{~min}$ at $4^{\circ} \mathrm{C}$ and resuspended in $40 \mathrm{~mL}$ of lysis buffer $(20 \mathrm{mM}$ Tris-HCl, $\mathrm{pH} 7.2,130 \mathrm{mM}$

$665 \mathrm{NaCl}, 10 \%$ glycerol, $1 \mathrm{mM}$ DTT, $1 \mathrm{mM} \beta$-mercaptoethanol supplemented with

666 phenylmethylsulfonyl fluoride (PMSF) and protease inhibitor cocktail (PIC; $31.2 \mathrm{mg}$

667 benzamide/mL, $0.5 \mathrm{mg}$ chymostatin $/ \mathrm{mL}, 0.5 \mathrm{~m}$ leupeptin $/ \mathrm{mL}, 0.1 \mathrm{mg}$ pepstatin $/ \mathrm{mL}, 1 \mathrm{mg}$

668 aprotonin/mL, $1 \mathrm{mg}$ antipain/mL). Cells were lysed by sonication and the lysate was cleared by 
669 centrifugation at $27,000 \times \mathrm{g}$ for $45 \mathrm{~min}$ at $4{ }^{\circ} \mathrm{C}$. H-NS containing cell lysate was directly applied to a HiTrap Heparin HP column (GE Healthcare) equilibrated with Binding Buffer with $130 \mathrm{mM}$

$671 \mathrm{NaCl}$. H-NS was eluted at $\sim 600 \mathrm{mM} \mathrm{NaCl}$ using a gradient elution from $130 \mathrm{mM}-1 \mathrm{M} \mathrm{NaCl}$

672 over 20 column volumes. Fractions containing H-NS were pooled and dialyzed against binding

673 buffer+130 mM NaCl. H-NS was applied to a 1-mL Resource Q column equilibrated with

674 Binding Buffer+130 mM NaCl. Remaining impurities were eluted over a gradient of 130-200

$675 \mathrm{mM} \mathrm{NaCl}$ in $10 \mathrm{~mL}$ of Binding Buffer. H-NS was eluted with $3 \mathrm{~mL}$ of Binding Buffer+500 mM

$676 \mathrm{NaCl}$. Fractions containing H-NS were dialyzed against H-NS Storage Buffer supplemented with

$6775 \mathrm{mM}$ TCEP. Purity ( $90 \%)$ and amount of protein $(\sim 4 \mathrm{mg})$ was determined by SDS-PAGE and

678 Qubit analysis, respectively. For tethered cleavage or $\mathrm{OH}$ cleavage assays, wild-type H-NS was 679 exchanged into a 3x stock of Filament Buffer (3x concentrations: $120 \mathrm{mM}$ HEPES-KOH, $\mathrm{pH}$ $680 \quad 8.0,300 \mathrm{mM}$ potassium glutamate, and 15\% glycerol).

\section{ChIP-seq sample preparation}

682 RL3000 cells (MG1655 F-, $\lambda-, i l v \mathrm{G} 468+, r f b-50, r p h+, y b h J(\mathrm{~L} 54->\mathrm{I}), y e b N(\mathrm{G} 25 \mathrm{xxx}->\mathrm{D})$, $y c f K:: 97 \mathrm{bp} \Delta I n s B-5 \Delta I n s A-5 \Delta I n s A B-5$; (Ghosh and Landick, 2016) were grown in $50 \mathrm{~mL}$ MOPS 684 defined minimal medium with 0.2\% glucose (GMM) (Neidhardt et al., 1974) at $37^{\circ} \mathrm{C}$ with vigorous shaking to an apparent $\mathrm{OD}_{600}$ of $\sim 0.4$ at which point either $100 \mu \mathrm{L}$ of $50 \mathrm{mg} \mathrm{Rif} / \mathrm{mL}$ in methanol (100 $\mu \mathrm{g}$ Rif/mL final) or $100 \mu \mathrm{L}$ of methanol as control and incubation at $37^{\circ} \mathrm{C}$ with shaking was continued for $30 \mathrm{~min}$. Cell preparation, crosslinking, and immunoprecipitation was completed as described previously (Mooney et al., 2009; Myers et al., 2013) with the following $\mathrm{mM}$ Tris, $\mathrm{pH}$ 8.0, $300 \mathrm{mM} \mathrm{NaCl}, 1 \%$ TritonX-100, $1 \mathrm{mM}$ PMSF), sonicated with the Misonix

691 Ultrasonic Liquid Processor (Model No. S-4000) for 1 hour at 60\% power, $10 \mathrm{sec}$ on and $10 \mathrm{sec}$ 692 off, and treated with $2 \mathrm{ng} \mathrm{RNase} \mathrm{A} / \mu \mathrm{L}$ (final concentration). To generate the input DNA control, 693 1/10th of the resulting lysate was removed and incubated with a Sepharose bead slurry (50-50 694 slurry of Sepharose Protein A and Protein G) overnight. The remaining lysate was pre-cleared 695 before adding the antibody by incubating the lysate with the Sepharose bead slurry at $4^{\circ} \mathrm{C}$ for 3 696 h. Beads were pelleted, and the cleared lysate was removed. Next, either $10 \mu \mathrm{L}$ of polyclonal H- 
NS antibody (Harlan Bioproducts) or $2 \mu \mathrm{L}$ Beta antibody (Neoclone \#WP0022; (Peters et al., 2012) were incubated with the lysate overnight. The next day, DNA/protein/antibody complexes were recovered with the Sepharose bead slurry and washed extensively before incubating at $65{ }^{\circ} \mathrm{C}$ for $30 \mathrm{~min}$ to reverse the crosslinks. Eluted DNA and input DNA (described above) were purified with Qiagen QIAquick PCR purification kit and eluted in $58 \mu \mathrm{L}$ of EB. DNA was quantified with Qubit dsDNA HS assay reagents (ThermoFisher). incubating $30 \mu \mathrm{L}$ of purified DNA from the IP with $0.4 \mathrm{mM}$ dNTPs (Fisher Scientific), $3 \mathrm{U} \mathrm{T} 4$ DNA polymerase, 1 U Klenow Enzyme, and 10 U T4 PNK in 1x T4 DNA ligase buffer (NEB) in a $50 \mu \mathrm{L}$ reaction for $30 \mathrm{~min}$ at $20^{\circ} \mathrm{C}$. DNA was then purified using Qiagen QIAquick PCR purification reagents. An A-overhang was added by incubating purified DNA with $1 \mathrm{mM} 2$ 'dATP and 5 U Klenow Exo 3', 5' minus (NEB \#M0212) in 1x NEBuffer 2 (\#B7002) in a $50 \mu \mathrm{L}$ reaction for $30 \mathrm{~min}$ at $37^{\circ} \mathrm{C}$. DNA was purified by using Qiagen MinElute PCR purification reagents and eluted in $10 \mu \mathrm{L}$ EB. Adapter and index oligo mixtures were made by incubating 30 $\mu \mathrm{M}$ universal adapter oligo and $30 \mu \mathrm{M}$ index primer in a $10 \mu \mathrm{L}$ reaction for $5 \mathrm{~min}$ at $100{ }^{\circ} \mathrm{C}$ and

712 slowly cooling back to room temp. Annealed oligos were ligated onto DNA fragments in a $30 \mu \mathrm{L}$ 713 reaction containing $10 \mu \mathrm{L}$ of purified DNA, $1 \mu \mathrm{L}$ annealed oligo mixture, $12 \mathrm{U}$ T4 DNA Ligase 714 (NEB), 1x DNA ligase buffer supplemented with $10 \mathrm{mM}$ ATP for $15 \mathrm{~min}$ at $20{ }^{\circ} \mathrm{C}$. DNA was 715 purified using Qiagen MinElute PCR purification reagents and resolved on a 2\% E-gel

716 (ThermoFisher). Fragments $\sim 270 \mathrm{bp}$ long were excised from the gel and purified by the 717 QIAquick gel extraction kit (Qiagen). $20 \mu \mathrm{L}$ DNA fragments were PCR amplified with $2 \mathrm{nM}$ 718 primers 1, 2, and the respective index primer using 2x Phusion High-fidelity PCR master mix 719 with HF buffer (NEB: M0531) (Table S2). Final PCR fragments were purified with QIAquick 720 PCR purification reagents. Quality and quantity of the finished libraries were assessed using an 721 Agilent DNA1000 chip and Qubit dsDNA HS Assay reagents, respectively. Libraries were 722 standardized to $2 \mathrm{nM}$. Cluster generation was performed using the Illumina cBot. Paired-end, 50723 bp sequencing was performed using standard sequencing by synthesis (SBS) chemistry on an 724 Illumina HiSeq2500 sequencer. Images were analyzed using the standard Illumina Pipeline, 725 version 1.8.2. 
727 Genomic DNA (gDNA) was isolated from stationary phase E. coli MG1655 cells using a

728 previously described CTAB isolation protocol (Wilson, 2001). Briefly, cells from an overnight

729 culture were diluted with TE (10 mM Tris-HCl pH 8.0, 1 mM EDTA) to an apparent OD 600 of 1

730 and final volume of $5 \mathrm{~mL}$ and then lysed by addition of $133 \mu \mathrm{L} 100 \mathrm{mg}$ lysozyme/mL at room

731 temperature for $5 \mathrm{~min}$. Proteins were removed from gDNA by incubating the solution with 267

$732 \mu \mathrm{L} 10 \% \mathrm{SDS}$ and $5.3 \mu \mathrm{L} 10 \mathrm{mg}$ proteinase $\mathrm{K} / \mathrm{mL}$ for 1 hour at $37{ }^{\circ} \mathrm{C}$. Polysaccharides were

733 removed from gDNA by adding $667 \mu \mathrm{L} 5 \mathrm{M} \mathrm{NaCl}$ and $667 \mu \mathrm{L} \mathrm{10 \%} \mathrm{CTAB} \mathrm{and} \mathrm{incubating} \mathrm{for} 10$

$734 \min$ at $65^{\circ} \mathrm{C}$. Remaining contaminants in the gDNA solution were removed by performing three

735 consecutive phenol:chloroform extractions where $3.3 \mathrm{~mL}$ of phenol:chloroform was added to the

$736 \quad 6.7 \mathrm{~mL}$ gDNA solution. DNA was then precipitated by addition of 0.6 volumes of chilled

737 isopropanol and pelleted. Precipitated DNA was resuspended in $100 \mu \mathrm{L}$ TE with $20 \mu \mathrm{g}$

738 RNase $\mathrm{A} / \mathrm{mL}$ and stored at $-20{ }^{\circ} \mathrm{C}$. Purity and concentration were determined with Nanodrop.

739 gDNA was sheared to fragments $\sim 200-700$ bp using the Misonix sonicator for 30 min at 60 watts

740 and $50 \%$ duty cycle.

741 To identify an optimal H-NS-DNA ratio for Nitro-seq that preferentially captured AT-rich DNA,

742 we tested different H-NS-gDNA ratios spiked with ${ }^{32} \mathrm{P}$-labeled AT-rich PCR fragments from the

$743 b g l$ operon and ${ }^{32} \mathrm{P}$-labeled GC-rich PCR fragments from pBR322. The sheared gDNA had an

744 average size of $\sim 880 \mathrm{bp}$ ) and the different H-NS-gDNA ratios were achieved by varying H-NS

745 concentration from 50 to $4000 \mathrm{nM}$ with $\sim 3.7 \mu \mathrm{M} \mathrm{bp} \cdot \mathrm{mL}^{-1}$ (equivalent to $\sim 500 \mu \mathrm{g} \cdot \mathrm{mL}^{-1}$ ) gDNA.

746 Radiolabeled DNA was prepared using OneTaq Polymerase (NEB), $250 \mathrm{nM}$ of the indicated

747 primers (Table S3), either genomic DNA, pMK110, or pBR322 as appropriate, and $2.5 \mu \mathrm{Ci}$ of

$748\left[\alpha-{ }^{32} \mathrm{P}\right] \mathrm{dCTP}$ per $50 \mu \mathrm{L}$ reaction. Excess label was removed using ProbeQuant G50 micro

749 columns (GE Healthcare \#28-9034-08). Nitro-seq reactions were performed in $250 \mu \mathrm{L}$ of

750 Filament Buffer (40 mM HEPES $\mathrm{KOH}, \mathrm{pH}$ 8.0, $100 \mathrm{mM}$ potassium glutamate, 5\% glycerol)

751 supplemented with $8 \mathrm{mM}$ magnesium aspartate containing gDNA (500 ng), $1 \mu \mathrm{L}$ of each PCR

752 product ( $\sim 50 \mathrm{ng}$ DNA or $\sim 8 \mathrm{nCi}$ ) and C-term His ${ }_{6}$-tagged H-NS at 50-4000 nM. Complexes were

753 formed for $20 \mathrm{~min}$ at $20^{\circ} \mathrm{C}$ before passing the mixture through a $0.45 \mu \mathrm{m}$ nitrocellulose filter

754 (Millipore CAS\# HAWP02400) at room temperature using Hoefer 10-well filtration apparatus 
755

756

757

758

759

760

761

762

763

764

765

766

767

768

769

770

771

772

773

774

775

776

777

778

779

780

781

782

(FH224V). Filters were washed twice with Filament Buffer containing 8 mM magnesium aspartate to remove non-specifically bound complexes. Bound DNA was eluted from the filters by incubating filters with $400 \mu \mathrm{L}$ of filter elution buffer $(20 \mathrm{mM}$ Tris- $\mathrm{HCl} \mathrm{pH} 7.8,0.2 \%$ sodium dodecyl sulfate, $0.3 \mathrm{M}$ sodium acetate) for 2 hours at $30{ }^{\circ} \mathrm{C}$ as previously described (Papoulas, 1996). Then, DNA was ethanol precipitated, separated by $6 \%$ urea-PAGE, and visualized with the Typhoon (GE Healthcare) (Figure $\boldsymbol{S 2}-1,2)$. Total ${ }^{32} \mathrm{P}$ applied to, retained by, and passed though the filter was determined by counting Cerenkov radiation. DNA eluted from the filters with $\sim 70 \%$ efficiency (Figure S2-1A). We found that $100 \mathrm{nM}$ and $200 \mathrm{nM} \mathrm{H}-\mathrm{NS}$ (31 and $64 \mathrm{H}-$ NS/kb gDNA, respectively) retained AT-rich fragments, but not GC-rich fragments (Figure S21). These H-NS-DNA ratios are similar to found previously to favor formation of bridged HNS-DNA filaments (Kotlajich et al., 2015). The optimization test also verified that short $(<1 \mathrm{~kb})$ DNA fragments, which are suitable for direct HT-sequence library preparation, were retained on the filter, indicating that they support H-NS-DNA filament formation. We found that longer fragments ( $\geq 959 \mathrm{bp}$ ) were preferentially retained on filters at lower H-NS concentrations (e.g. 50 nM H-NS; 15 H-NS/kb) than shorter ( $\leq 630$ bp) filaments (Figure $\boldsymbol{S} 2-1)$. We chose $100 \mathrm{nM}$ and $200 \mathrm{nM} H-N S$ (31 and 64 H-NS/kb DNA) for subsequent experiments because these conditions gave selective retention of AT-rich fragments short enough for use in HT-sequencing reactions.

A similar optimization was performed for StpA and for Fis. For StpA, 50 or $100 \mathrm{nM}$ StpA retained AT-rich but not GC-rich PCR fragments with selectivity similar to $100 \mathrm{nM} \mathrm{H}-\mathrm{NS}$. For Fis, we used different radiolabeled PCR fragments containing known Fis-binding sites (Kahramanoglou et al., 2011) (Table S3). We found that 5 and 12 nM Fis retained Fis-specific PCR fragments on the nitrocellulose filter (Figure S2-2). No optimization was performed for Hha-H-NS filaments because they are known to form at 1:1 Hha:H-NS ratio (Ali et al., 2013). Thus, we added equimolar Hha to an H-NS concentration known to retain AT-rich fragments (100 nM).

For Nitro-seq experiments, H-NS, StpA, or Fis were mixed with 500 ng sheared gDNA at concentrations that selected AT-rich DNA fragments, but not GC-rich fragments (100 and 200 nM H-NS; 50 and 100 nM StpA; 5 and 12 nM Fis; Figure 2A; Figure S2-1,2) as described 
783

784

785

786

787

788

789

790

791

792

793

794

795

796

797

798

799

800

801

802

803

804

805

806

807

808

809

810

above. Protein-DNA complexes were isolated as described above for the optimization experiments except DNA was resuspended in water. Total gDNA applied to, retained by, and passed though the filters was determined using Qubit dsDNA HS Assay reagents (Figure S2-1). The percentage of gDNA retained by H-NS in our Nitro-seq experiments $(\sim 10 \%)$ was similar to the percentage of the $E$. coli K-12 genome bound by H-NS in vivo (15\%) (Grainger et al., 2006; Kahramanoglou et al., 2011; Myers et al., 2013; Navarre et al., 2006), which further supports our Nitro-seq conditions as being appropriate to determine the DNA sequence specificities of bacterial chromatin proteins. DNA sequencing libraries were prepared for each sample using NEBNext Ultra II DNA library reagents (NEB \#E7103). The quality and quantity of libraries and sequencing was performed like the ChIP-seq experiment described above except cluster generation was not performed.

\section{ChIP-seq and Nitrocellulose-seq sequence processing and alignment}

Sequencing adapter reads and reads shorter than $30 \mathrm{nt}$ were removed with Trimmomatic (version 0.) (Bolger et al., 2014) and the quality of remaining read were confirmed by FastQC (Andrews, 2010). Remaining paired-end reads were aligned to the MG1655 genome (Accession: NC_000913.3) using Bowtie2 (version 2.3.4.2) with the parameters (-q --end-to-end -I 100 -X 800 --fr -t) (Langmead and Salzberg, 2012). Correctly paired reads were retained and duplicate reads were removed with Samtools (version 1.9)(Li et al., 2009) and bedgraph files for visualization were created with BEDtools (version 2.8) (Quinlan and Hall, 2010). More than 95\% of reads mapped properly to the genome for ChIP-seq (Table S4) and Nitro-seq experiments (Table S7) and were of high quality.

\section{ChIP-seq and Nitro-seq data analysis}

ChIP- or Nitro-seq patterns, read coverage in each condition was scaled for sequencing depth by reads per million. To correct ChIP-seq patterns for variations in amounts of input DNA and in sequencing, the $\log _{2}$ of control reads from input DNA was subtracted from the $\log _{2}$ of IP reads to give a corrected read coverage. The RNAP baseline was set to the ChIP signal level in regions known to be transcriptionally silenced (Mooney et al., 2009) and the H-NS baseline was set at the average IP/input values from at least 5 regions with little signal. A similar analysis was 
811 performed for Nitro-seq samples by correcting the nitrocellulose retained reads using input reads.

812 When compared, $\log _{2}$ (ChIP/input) or $\log _{2}$ (Nitro/input) samples were first quantile normalized

813 together using R. To compare Nitro-seq patterns, the genome was divided into 200 bp bins and

814 the average $\log _{2}$ (Nitro/input) was calculated for each bin using a custom script. The density of

815 read coverage in each bin was compared between samples used to calculate Spearman

816 correlation coefficients (Figure S3-1).

817 To determine the enriched DNA segments more rigorously, we used MOSAiCS (Model-

818 based one and two Sample Analysis and Inference for ChIP-Seq Data) (Kuan et al., 2011),

819 specifically the two-sample analysis and MOSAiCS with a hidden Markov Model (MOSAiCS-

820 HMM) (Chung et al., 2014). This approach allowed determination of enriched regions for an IP

821 or nitrocellulose-retained sample compared to a matched input sample. For H-NS and RNAP

822 ChIP, 200-bp bins were used and duplicate reads were capped at 5 before fitting to a robust

823 background model using MOSAiCS-HMM. Using the viterbi algorithm, enriched regions were

824 determined using a false discovery rate $(F D R) \leq 0.05$. Enriched region boundaries were adjusted,

825 and false positive peaks were filtered out using default settings in the MOSAiCS package.

826 Enriched regions present in both H-NS ChIP replicates were determined using BEDtools

827 “intersect” using default parameters to assign the enriched regions for cells with or without Rif-

828 treated cells (Quinlan and Hall, 2010). The H-NS binding patterns in the untreated cells was

829 similar to those reported using ChIP-chip in strain MG1655 also grown in GMM (Myers et al.,

830 2013) and to the ChIP-seq patterns reported for flag-tagged H-NS in MG1655 grown in broth

831 (LB 0.5\% NaCl; Figure S1-1C; (Kahramanoglou et al., 2011), confirming our analysis of H-NS

832 filaments in vivo. For the RNAP ChIP in Rif-treated cells, settings similar to untreated RNAP

833 ChIP were used find enriched regions, but using a non-HMM MOSAiCS fit for background and

834 mosaicsPeak parameters of $\mathrm{FDR}=0.05$, maxgap $=200$, minsize $=50$, and thres $=10$. For Nitro-seq,

835 MOSAiCS analysis was performed as described for H-NS ChIP-seq except that bins were

836 created without removing duplicates and enriched regions were identified using the posterior

837 decoding algorithm. Nitro-seq enriched regions were defined as those identified in at least two of

838 the three replicates. The features of MOSAiCS-defined regions for each dataset are summarized

839 in Table 1. 
To compare MOSAiCS-identified regions between conditions, we used BEDtools (Quinlan

841 and Hall, 2010). First, we identified regions that overlapped between conditions using the

842 BEDtools "intersect" with options set to report the original regions (-wa -wb). A scatterplot of

843 the lengths of these regions was generated using Excel. The complete regions or fractions of

844 regions bound in only one condition were determined using BEDtools "subtract" with default

845 parameters. The similarity between two conditions was calculated as the Jaccard index using the

846 BEDtools "jaccard" command, which calculates the percentage of the length of filaments that

847 overlap out of the total length of filaments that do not overlap (Favorov et al., 2012). Thus,

848 completely dissimilar, non-overlapping data sets have a Jaccard index of $0 \%$ is whereas a

849 Jaccard index of $100 \%$ indicates that two datasets completely overlap.

To assign regions differentially bound by H-NS, the average ChIP signal from both

851 replicates in each condition was calculated using a custom script. The difference in the $\log _{2}$ ratio

852 of untreated H-NS signal and Rif-treatment $\mathrm{H}-\mathrm{NS}$ signal $\geq 0.15$ was used to assign regions of $\mathrm{H}$ -

853 NS depletion upon Rif treatment whereas a ratio $\leq-0.15$ was used to assign regions of $\mathrm{H}-\mathrm{NS}$

854 enrichment upon in Rif treatment (Table S5). Similar cutoffs were used for RNAP ChIP to

855 determine differences in RNAP occupancy in H-NS differentially bound regions. A correlation

856 between an H-NS differentially bound region and a change in RNAP binding was confirmed if

857 either MOSAiCS or average ChIP signal showed a change in RNAP binding at that H-NS

858 differentially bound region.

859 To compare H-NS filaments found only in vitro (Nitro-seq specific) to known TF binding

860 sites, we calculated the overlap between H-NS filaments and regions of TF-binding described in

861 RegulonDB (Santos-Zavaleta et al., 2019) with Bedtools "intersect". The overlap between Nitro-

862 seq specific regions with tsEPODs and heEPODs (Vora et al., 2009) also was calculated with

863 Bedtools "intersect". To calculate the $p$-value for these overlaps, the overlap between known

864 protein binding sites and 112 randomized sites of the same length as the 112 Nitro-seq specific

865 sites was determined 1000 times. 


\section{FeBABE Conjugation}

H-NS C21S and H-NS C21S E102C were exchanged into Conjugation Buffer (10 mM MOPS, pH 8.0, 0.5 mM EDTA, 200 mM NaCl, 5\% glycerol) using PD SpinTrap G-25 columns (GE Healthcare \#28-9180-04) pre-equilibrated with conjugation buffer. FeBABE was charged by incubating (S)-1-(p-Bromoacetamidobenzyl)ethylenediaminetetraacetate (BABE; Santa Cruz Biotechnology) with $\mathrm{FeCl}_{3} \bullet 6 \mathrm{H}_{2} \mathrm{O}$ as previously described (Greiner et al., 1997). H-NS variants $(50 \mu \mathrm{M})$ were incubated with 10 -fold molar excess FeBABE for $2 \mathrm{~h}$ at room temperature to conjugate FeBABE to H-NS. After conjugation, 7.6 $\mu$ mol of conjugated H-NS was combined with 20-fold-molar excess mPEG24 (ThermoFisher \# 22713) for an additional $2 \mathrm{~h}$ at room temperature. Samples were visualized on 10\% Bis-tris gel (ThermoFisher) to determine the percent conjugated ( $\sim 50 \%$ for H-NS C21S E102C). Excess FeBABE was removed by applying conjugated H-NS to a clean PD SpinTrap G-25 column equilibrated with a $3 \mathrm{X}$ stock of Filament Buffer. Conjugation was also confirmed by the appearance of a second, slower migrating band on $20 \%$ PhastGel (GE Healthcare). Fe·EDTA-H-NS was stored in aliquots at $-80^{\circ} \mathrm{C}$ and remained stable under these storage conditions for up to 1 year. The DNA-binding activity of the conjugated protein was confirmed by electrophoretic mobility shift assay and by in vitro transcription assays described previously (Figure S4-2,3) (Boudreau et al., 2018; Kotlajich et al., 2015).

\section{Tethered-nuclease mapping (TEN-map) Assay}

The 146-bp DRE DNA for the TEN-map assay was generated by PCR from a plasmid pMK110 using primers \#7486 and \#12638 (Tables S1 and S2). To enable visualization by phosphorimaging, one primer was 5 '-end-labeled with $\left[\gamma-{ }^{32} \mathrm{P}\right] \mathrm{ATP}$ prior to the PCR reaction. The PCR product was separated on 5\% non-denaturing PAG, eluted from the gel overnight at $4{ }^{\circ} \mathrm{C}$ in $20 \mathrm{mM}$ Tris- $\mathrm{HCl}$ pH 7.5, 1 mM EDTA, and $200 \mathrm{mM} \mathrm{NaCl}$, and concentrated using QIAquick gel extraction kit. Increasing concentrations of Fe·EDTA-H-NS (from 50 to $5000 \mathrm{nM}$ ) were 891 incubated with $\sim 18 \mathrm{nM}$ bgl DRE DNA for $20 \mathrm{~min}$ at $20^{\circ} \mathrm{C}$ in $7.5 \mu \mathrm{L}$ of $1 \mathrm{X}$ Filament Buffer 892 supplemented with $8 \mathrm{mM}$ magnesium aspartate. Hydroxyl radical cleavage was initiated by 893 addition of $1 \mu \mathrm{L} 2 \mathrm{mM} \mathrm{H}_{2} \mathrm{O}_{2}$ and $1 \mu \mathrm{L} 2 \mathrm{mM}$ ascorbic acid. Cleavage proceeded for $2 \mathrm{~min}$ at 20 
${ }^{\circ} \mathrm{C}$ after which $8 \mu \mathrm{L}$ of the cleavage reaction was added to $100 \mu \mathrm{L}$ TE-saturated phenol, $\mathrm{pH} 7.9$ and $92 \mu \mathrm{L}$ cleavage stop solution (20 mM EDTA, $20 \mu \mathrm{g}$ glycogen) were added and samples were vortexed for $10 \mathrm{sec}$. Samples were centrifuged to separate layers and $90 \mu \mathrm{L}$ of the aqueous layer was added to $280 \mu \mathrm{L}$ of $100 \mu \mathrm{L}$ ethanol to precipitate DNA. Samples were washed twice with $500 \mu \mathrm{L}$ of $70 \%$ ethanol and resuspended in $4 \mu \mathrm{L}$ of formamide stop dye (95\% formamide, 20 mM EDTA, $0.02 \%$ xylene cyanol, $0.02 \%$ bromophenol blue). The samples were electrophoresed through a $7 \%$ urea polyacrylamide gel $(40.4 \times 45.7 \mathrm{~cm})$ in $0.5 \mathrm{X}$ TBE and alongside a Maxam-

901 Gilbert A+G ladder for alignment (Maxam and Gilbert, 1977) and MspI-digested, ${ }^{32} \mathrm{P}-$-end-

902 labeled pBR322 for size reference. The cleaved DNA was quantified using semi-automated

903 footprinting analysis (SAFA) (Das et al., 2005). The resulting peak areas were first normalized to 904 total signal in each sample as determined by ImageQuant (version 5.2; GE Lifesciences) analysis 905 of an unsaturated phosphorimage scan of gel and then adjusted to values above and below the 906 average peak area in each sample.

907 For the offset DNA experiment (Figure S4-7), the TEN-map assay was performed as 908 described above except using a 151-bp bgl DRE DNA amplified from pMK110 using 5' ${ }^{32}$ P-end909 labeled primer \#14374 and unlabeled primer \#12386. For the TEN-map assay on the 549-bp bgl 910 DRE fragment (Figure 5), DNA was amplified from pMK110 using 5' ${ }^{32} \mathrm{P}$-end-labeled primer 911 \#7486 and unlabeled primer \#7491. A mixture of Fe·EDTA-H-NS and wild-type H-NS at 4:6 912 ratio and concentration indicated in the figure legend was added to $\sim 18 \mathrm{nM}$ DNA in a $20 \mu \mathrm{L}$ 913 reaction in Filament Buffer supplemented with $0.02 \%$ NP-40 and $100 \mu$ g acetylated BSA $/ \mathrm{mL}$ 914 (supplemented Filament Buffer) to compare to the bridged filament cleavage described below.

915 Cleavage was initiated by addition of $1 \mu \mathrm{L} 50 \mathrm{mM}$ ascorbic acid and $4 \mu \mathrm{L} 12.5 \mathrm{mM} \mathrm{H}_{2} \mathrm{O}_{2}$.

916 Cleavage continued for $2 \mathrm{~min}$ at RT before stopping the reaction with $25 \mu \mathrm{L} 22.4 \mathrm{mM}$ thiourea

917 and $20 \mu \mathrm{L}$ TE. The cleavage products were recovered by phenol extraction and ethanol

918 precipitation, and then resuspended in $4 \mu \mathrm{L}$ formamide stop dye. The cleavage products were

919 visualized and quantified as described above for the 146-bp $b g l$ DRE DNA. To test the effect of $920 \mathrm{Mg}^{2+}$ on the H-NS DBD location and orientation (Figure $5 \boldsymbol{B}$ ), the TEN-map assay was 921 performed on the $549 \mathrm{bp}$ DNA fragment as described except the amount of magnesium aspartate 922 was varied as specified in the figure legend. 
923 Hydroxyl radical footprinting

924 H-NS filaments were formed on the same 146-bp $b g l$ DRE DNA template used in the TEN-map 925 assay. Concentrations of unmodified H-NS from $50 \mathrm{nM}$ to $4 \mu \mathrm{M}(17-1360 \mathrm{H}-\mathrm{NS} / \mathrm{kb})$ were 926 incubated to $\sim 20 \mathrm{nM} \mathrm{5}{ }^{\prime 32} \mathrm{P}$-end-labeled $b g l$ DRE DNA in $50 \mu \mathrm{L}$ reactions in Filament Buffer

927 without glycerol. Filaments were allowed to form at $20^{\circ} \mathrm{C}$ for $20 \mathrm{~min}$ prior to $\mathrm{OH}$ cleavage.

928 Cleavage was initiated by addition of $0.6 \mu \mathrm{L} 100 \mathrm{mM}$ ascorbic acid, $4 \mu \mathrm{L} 0.15 \% \mathrm{H}_{2} \mathrm{O}_{2}$, and $6 \mu \mathrm{L}$ 929 fresh $50 \mathrm{mM} \mathrm{Fe}^{2+}$-EDTA $\left(50 \mathrm{mM}\left(\mathrm{NH}_{4}\right)_{2} \mathrm{Fe}\left(\mathrm{SO}_{4}\right)_{2}, 100 \mathrm{mM}\right.$ EDTA) and incubated for $2 \mathrm{~min}$ at

$93020^{\circ} \mathrm{C}$. The no-cleavage control was treated identically except $10.6 \mu \mathrm{L}$ water was added in place 931 of cleavage reagents. $\cdot \mathrm{OH}$ was then quenched by addition of $80 \mu \mathrm{L} 20 \mathrm{mM}$ thiourea and $60 \mu \mathrm{L}$

932 of $0.3 \mathrm{M} \mathrm{NaCl}, 1 \mathrm{mM}$ EDTA. Cleavage products were recovered by phenol extraction and 933 ethanol precipitation, and then resuspended in $4 \mu \mathrm{L}$ formamide stop dye. Samples visualized as 934 described for TEN-map assay and quantified using SAFA (Das et al., 2005).

\section{Bridged H-NS cleavage assay}

936 Bridged H-NS filaments were formed using the previously published bridging pull-down assay 937 (van der Valk et al., 2018; van der Valk et al., 2017), with the following modifications. The 5' 938 32P-end-labeled, 549-bp bgl DRE DNA (see above) was used as the prey DNA. To form bridged filaments, $5 \mathrm{nM}$ biotinylated 685-bp DNA fragment (68\% AT; Table S2) coupled to $3 \mu \mathrm{L}$ M280

940 streptavidin dynabeads (ThermoFisher) was incubated with $5 \mathrm{nM}$ prey DNA and H-NS in 941 supplemented Filament Buffer at 5-8 $\mu \mathrm{M}$ 6:4 WT H-NS: Fe·EDTA-H-NS as indicated in the

942 legend to Figure 5. High levels of H-NS $(>5 \mu \mathrm{M})$ are required to form bridged filaments in the 943 bridging/pull-down assay, possibly because DNA sequestration on the positively charged 944 streptavidin beads inhibits H-NS binding (Figure S5-2B; (van der Valk et al., 2017). Bridged

945 filaments were formed for $20 \mathrm{~min}$ at $20^{\circ} \mathrm{C}$ with shaking at $1000 \mathrm{rpm}$ in an Eppendorf

946 Thermomixer and then separated from unbound protein and DNA using a magnetic rack. Bead-

947 immobilized bridged filaments were then resuspended in $20 \mu \mathrm{L}$ supplemented Filament Buffer

948 followed by addition of $1 \mu \mathrm{L} 50 \mathrm{mM}$ ascorbic acid and $4 \mu \mathrm{L} 12.5 \mathrm{mM} \mathrm{H}_{2} \mathrm{O}_{2}$. Cleavage was

949 allowed to proceed for $2 \mathrm{~min}$ at RT before addition of $25 \mu \mathrm{L} 22.4 \mathrm{mM}$ thiourea and $20 \mu \mathrm{L}$ TE.

950 DNA recovered and resuspended in formamide stop dye as described above. The amount of ${ }^{32} \mathrm{P}$ 
951 retained on the beads was measured by counting Cerenkov radiation and compared to amounts

952 retained using only unmodified H-NS and prey DNA. Bridging efficiency was calculated as a

953 fraction of total prey DNA ${ }^{32} \mathrm{P}$ that was retained on the beads. Apparent bridging efficiency was

954 likely decreased by some DNA lost during sample processing. Cleavage products were then

955 visualized and quantified by gel electrophoresis and phosphorimaging as described above for the

956 TEN-map assay.

\section{MD simulation of FeBABE-H-NS bound to DNA}

958 Molecular dynamics simulations were run using the program OpenMM (Eastman et al., 2017)

959 with the Charmm36 force field (Best et al., 2012; Hart et al., 2012). Initial setup and post-

960 processing analyses were conducted using the program CHARMM (Brooks et al., 2009). We

961 obtained an initial starting model of H-NS bound to a 12-bp strand of duplex DNA from Vreede

962 and coworkers (Riccardi et al., 2019). We "mutated" the side-chain of Cys 102 in that model to

963 an FeBABE moiety and generated force field parameters for the organic component of FeBABE

964 by analogy to existing functional groups in the Charmm36 force field. We modeled the $\mathrm{Fe}^{2+}$

965 using a cationic dummy-atom model (Liao et al., 2017). We dissolved the H-NS/FeBABE/DNA

966 system in a cubic box of water with $70 \AA$ edges and added $\mathrm{Na}^{+}$and $\mathrm{Cl}^{-}$ions to reach an ionic

967 strength corresponding to $150 \mathrm{mM}$ with neutral overall charge. The complete system contained

96831,518 atoms. Following a short geometry optimization, the system was heated from $48 \mathrm{~K}$ to

$969298 \mathrm{~K}$ during $1 \mathrm{~ns}$ and equilibrated at $298 \mathrm{~K}$ and $1 \mathrm{~atm}$ for an additional $4 \mathrm{~ns}$ prior to production

970 simulations. The simulations employed the NPT ensemble using the Andersen thermostat and

971 MC barostat. Non-bonded interactions were cutoff above a distance of $12 \AA$ with a switching

972 function from $10 \AA$ to $12 \AA$ and the integration time step was 1 fs. We placed a harmonic

973 restraint with force constant of $1 \mathrm{kcal} / \mathrm{mol} \bullet \mathrm{A}^{2}$ on DNA backbone atoms to prevent unraveling at

974 the ends of the duplex, which we observed in initial tests. Under these conditions, we simulated

975 the system for $500 \mathrm{~ns}$, taking snapshots every 10 ps.

976 In each frame, we calculated the distance from the $\mathrm{Fe}^{2+}$ to every $\mathrm{C}^{\prime}$, because FeBABE-

977 induced cleavage is thought to occur by $\cdot \mathrm{OH}$ radical reacting with $\mathrm{C} 5{ }^{\prime}-\mathrm{H}$ (Balasubramanian et

978 al., 1998). We modeled the concentration of $\cdot \mathrm{OH}$ radicals at $\mathrm{C}^{\prime}$ ' based on a diffusion model 
979 where the concentration of radicals around the $\mathrm{Fe}^{2+}$ decays according to a Gaussian distribution

980 (Cheal et al., 2009). To account for cleavage sites that are shielded from solvent by the bound

981 protein, the average concentration of $\cdot \mathrm{OH}$ at each site was scaled linearly by the average solvent

982 accessible surface area of the corresponding C5' methylene group (Figure $\boldsymbol{S} 7-2 \boldsymbol{A})$. The scaled

983 concentration at each site yields the cleavage propensities displayed in Figure $7 \boldsymbol{A}$. The

984 simulations used a relatively short 12-bp duplex in order to achieve reasonable computational

985 efficiency and to run simulations long enough to adequately sample the conformational

986 flexibility of the FeBABE. To model cleavage propensities for bp at greater distances from the

987 H-NS binding site, we assumed that long-range interactions with DNA do not influence the

988 overall behavior of FeBABE and thus estimated those cleavage propensities by adding additional

989 DNA to the saved trajectories after the simulation was complete.

\section{Modeling of DBDs in H-NS filaments}

991 The $\cdot \mathrm{OH}$ footprinting data for each $\mathrm{H}-\mathrm{NS}$ concentration tested ( $0-4 \mu \mathrm{M}$ titration), each strand,

992 and each independent replicate was normalized by dividing the signal in each lane by the average

993 signal within a lane. The resulting ratio was $\log _{2}$ transformed to center the data at zero. The $\log _{2}-$

994 normalized deviance from the average signal from each strand was separately fit to a Bayesian

995

996

997

998

999

1000

1001

1002

1003

1004

1005

1006

multi-level model of the following form:

$$
\begin{gathered}
y_{i} \sim \text { Student's } t\left(\mu_{i}, \sigma, v\right) \\
\mu_{i}=\alpha_{\mathrm{bp}[i]}+\beta_{\mathrm{bp}[i]}[\mathrm{HNS}] \\
{\left[\begin{array}{c}
\alpha_{\mathrm{bp}} \\
\beta_{\mathrm{bp}}
\end{array}\right]=\operatorname{MVNormal}\left(\left[\begin{array}{l}
\alpha \\
\beta
\end{array}\right], \boldsymbol{S}\right)} \\
\boldsymbol{S}=\left(\begin{array}{cc}
\sigma_{\alpha} & 0 \\
0 & \sigma_{\beta}
\end{array}\right) \boldsymbol{R}\left(\begin{array}{cc}
\sigma_{\alpha} & 0 \\
0 & \sigma_{\beta}
\end{array}\right) \\
\boldsymbol{R}=\left(\begin{array}{ll}
1 & \rho \\
\rho & 1
\end{array}\right)
\end{gathered}
$$

With the following weakly regularizing priors:

$$
\begin{gathered}
\alpha \sim \operatorname{Normal}(0,10) \\
\beta \sim \operatorname{Normal}(0,10) \\
\sigma \sim \operatorname{HalfCauchy}(0,1) \\
\sigma_{\alpha} \sim \operatorname{HalfCauchy}(0,1)
\end{gathered}
$$




$$
\boldsymbol{R} \sim \operatorname{LKJ} \operatorname{corr}(2)
$$

1009 The models were fit using the R package brms (version 2.10.0) (Bürkner, 2017) and default 1010 priors were used for any parameter for which a prior is not specified above.

1011 Similarly, the tethered-cleavage data for each concentration (0-3 $\mu \mathrm{M}$ titration) was

1012 normalized by first dividing each lane by a constant factor resulting from quantification of a

1013 short scan of the entire lane. The data was then centered by subtracting the average signal within

1014 each lane. The centered and normalized signal for each strand separately was then fit to a

1015 Bayesian multilevel, of the form described above, with the following priors:

1016

1018

$$
\begin{aligned}
& \alpha \sim \operatorname{Normal}(0,0.1) \\
& \beta \sim \operatorname{Normal}(0,0.1)
\end{aligned}
$$

Default priors were used for every parameter not specified above. Notably, the $4 \mu \mathrm{M}$ and $5 \mu \mathrm{M}$ concentrations were not included in the tethered cleavage fits due to their substantial deviance from linearity. Point estimates for each slope at each bp in the models above were estimated from the average of the posterior distribution for that slope. These estimates can be interpreted as the H-NS concentration-dependent change in signal at each bp with data-driven shrinkage towards the grand mean of slopes for every $\mathrm{bp}$.

To match the MD simulations with the in vitro data, the slope estimates for each strand in each data type were Robust- $Z$ normalized using the following formula:

$$
y_{\text {scaled }}=\frac{y-\operatorname{median}(y)}{1.4826 \times \operatorname{MAD}(y)}
$$

where MAD is the median absolute deviation and 1.4826 is a scaling factor to convert the MAD to an estimate for the standard deviation (Hampel, 1974; Rousseeuw and Croux, 1993). Similarly, the expected 15-bp cleavage pattern of a single H-NS DBD (Figure 7A) was also scaled to a standard Normal using the formula above. Using a sliding window, the Manhattan distance between the scaled, tethered-cleavage estimates and the scaled, 15-bp expected cleavage pattern was calculated for each base centered on the sixth bp of the cleavage pattern (triangle in Figure $7 \boldsymbol{B}$ ). Due to the asymmetry of the expected cleavage pattern, we performed this calculation in both directions along the DNA corresponding to two different possible orientations 
1035 of the H-NS DNA binding domain. Since this score is a distance, lower values indicate a closer

1036 match between the expected cleavage pattern and the tethered cleavage data.

1037 To assess the statistical significance of the MD match scores, we performed this same

1038 procedure on 1000 different random shuffles of the tethered-cleavage data for each direction of

1039 the H-NS DBD. We considered MD match scores below the $5^{\text {th }}$ percentile of the random MD

1040 match scores to signify a statistically significant match between the expected cleavage pattern

1041 and the tethered cleavage data.

1042 To determine possible localizations of the H-NS DBD in the $\cdot \mathrm{OH}$ footprinting data, we

1043 performed the same procedure described above using the solvent accessibility of the $5^{\prime}$ carbon of

1044 the DNA duplex as the expected pattern of protection imparted by the H-NS DBD (Figure S7-

$10452 \boldsymbol{A}$ ) and matched that pattern to the $\cdot \mathrm{OH}$ footprinting data (Figure S6-3).

1046 Predicting H-NS binding patterns genome-wide

1047 To generate a dataset for genome-wide prediction of the locations of H-NS filaments, the E. coli

1048 genome (accession U00096.3) was divided into a tiling array of 1000-bp windows staggered

1049 every $250 \mathrm{bp}$. A window was considered to be within a peak if over $50 \%$ of the window was

1050 covered by a called peak for that dataset. Any window that did not meet this criterium was

1051 considered as part of the unbound negative set. For each window the following sequence features

1052 were generated: a count of the As and Ts (A/T content), a count of 4mers matching the criteria

1053 for an AT tract, a count of 4mers matching the criteria for a TA step, a count of motif matches to

1054 the Lang et al. H-NS PWM (Lang et al., 2007), and a count of motif matches to the RegulonDB

1055 (Santos-Zavaleta et al., 2019) Fis motif. Motif matches were generated by running FIMO

1056 (version 5.05) (Grant et al., 2011) over the entire E. coli genome with a third-order Markov

1057 background model, searching on both strands, and using a $p$-value cutoff of 0.001 with no FDR

1058 correction. Each of these counts was then normalized by the length of the window. DNA

1059 structure features were generated using the DNAshapeR package (Chiu et al., 2016) run over the

1060 entire E. coli genome. The MGW, ProT, and EP bp level features were then Robust-Z

1061 normalized using data from the entire genome and the number of bases below a -2 cutoff on the

1062 Robust-Z scale for each feature was determined and normalized by the length of the window. 
To predict genome-wide locations of H-NS filaments, a LASSO logistic regression model

1064 was trained on the H-NS ChIP data using glmnet version 2.0-16 (Friedman et al., 2010). All

1065 features were centered and scaled before training. The LASSO model reveals the minimal set of

1066 DNA features needed for the prediction of a given dataset by only including non-zero

1067 coefficients for a feature if that feature provides independent information from other non-zero

1068 coefficients already present in the model. Since all input data was centered and scaled before

1069 training the model, the relative magnitude of each coefficient can be interpreted as the

1070 importance for that feature in predicting the outcome, with positive values indicating an increase

1071 in the probability of a region being bound by a protein when the associated feature is a higher

1072 value. Models were trained using 10-fold cross-validation with stratified sampling and the

1073 penalty parameter, $\lambda$, set as one standard error away from the minimum binomial deviance. $\lambda$

1074 was determined individually for each subsampled dataset using 10-fold cross-validation

1075 internally to test for sensitivity to the exact data used to train the model. Predictive performance

1076 for each ChIP and Nitro-seq dataset was tested by calculating the area under the precision-recall

1077 curve (AUC-PRC) using the method of Davis and Goadrich (2006) with the R package PRROC

1078 version 1.3.1 (Grau et al., 2015). The average, minimum, and maximum AUC-PRC was

1079 determined using the fitted models for each subsampled dataset to predict the binding locations

1080 for each ChIP and Nitro-seq dataset.

1081 Data availability

1082 Raw sequencing data, processed bedgraph, and bed files from the MOSAiCS output have been

1083 deposited in the Gene Expression Omnibus with accession number GSE157512. Custom scripts

1084 for ChIP-seq and Nitro-seq analysis are available at https://github.com/jmvera255/bio-script-

1085 library/tree/master/perl.

\section{ACKNOWLEDGEMENTS}

1087 We thank Jocelyne Vreede (University of Amsterdam) for sharing coordinates for an H-NS

1088 DBD-DNA structure and for modeling FeBABE into the structure; Wilma Ross for providing

1089 purified Fis, advice on footprinting experiments, and equipment for Nitro-seq experiments;

1090 Jessica Vera for sharing scripts to analyze ChIP-seq and Nitro-seq data; Peter Freddolino for 
1091 suggestions of training sets for DBPs that bind a large fraction of the genome; and members of

1092 the Landick lab for many helpful discussions and comments on this manuscript. This work was

1093 supported by NIH grant GM38660 to R.L., Chemical Biology Interface Training grant (NIH T32

1094 GM008505) to C.M.H., and the National Science Foundation Graduate Research Fellowship

1095 under grant number DGE-1256259 to B.A.S. and DGE-1747503 to C.M.H. High-throughput

1096 sequencing services were provided by the University of Wisconsin Biotechnology Center DNA

1097 Sequencing Facility. Computational resources for MD simulations were provided by the Extreme

1098 Science and Engineering Discovery Environment (XSEDE; allocation TG-MCB180084 to D.R.),

1099 which is supported by National Science Foundation grant number ACI-1548562.

1100 
1101 TABLES

1102 Table 1. Enriched ChIP-seq and Nitro-seq regions identified by MOSAiCS

\begin{tabular}{|c|c|c|c|c|c|c|c|}
\hline SAMPLE & METHOD & $\begin{array}{l}\text { TOTAL } \\
\text { REGIONS }\end{array}$ & $\begin{array}{l}\text { AVERAGE } \\
\text { LENGTH }\end{array}$ & $\begin{array}{l}\text { MEDIAN } \\
\text { LENGTH }\end{array}$ & $\begin{array}{l}\text { SMALLEST } \\
\text { REGION }\end{array}$ & $\begin{array}{l}\text { LARGEST } \\
\text { REGION }\end{array}$ & FDR \\
\hline Untreated & H-NS ChIP & 301 & 2005 & 1221 & 220 & 15629 & 0.02 \\
\hline Rif Treated & H-NS ChIP & 289 & 2059 & 1279 & 211 & 15486 & 0.03 \\
\hline Untreated & Beta ChIP & 294 & 1829 & 875 & 94 & 22190 & 0.06 \\
\hline Rif Treated & Beta ChIP & 1352 & 601 & 400 & 200 & 3400 & 0.05 \\
\hline $100 \mathrm{nM}$ H-NS & Nitro & 407 & 2066 & 1436 & 242 & 14836 & 0.05 \\
\hline 200 nM H-NS & Nitro & 380 & 1902 & 1300 & 176 & 15281 & 0.05 \\
\hline 50 nM StpA & Nitro & 589 & 1817 & 1241 & 176 & 15281 & 0.05 \\
\hline 100 nM StpA & Nitro & 325 & 2217 & 1542 & 117 & 1600 & 0.05 \\
\hline Hha:H-NS & Nitro & 436 & 2056 & 1490.5 & 122 & 14881 & 0.05 \\
\hline $12 \mathrm{nM}$ Fis & Nitro & 888 & 917 & 818 & 209 & 4207 & 0.05 \\
\hline
\end{tabular}

1103

1104 


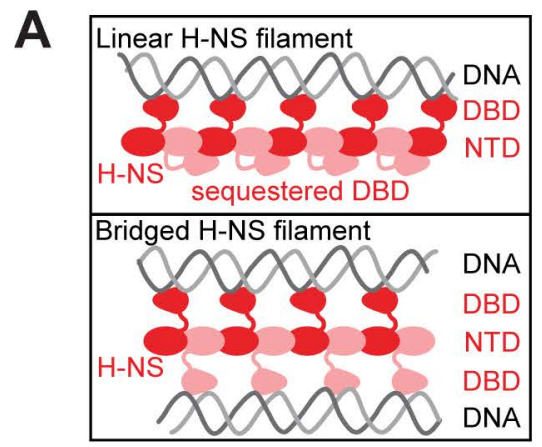

B

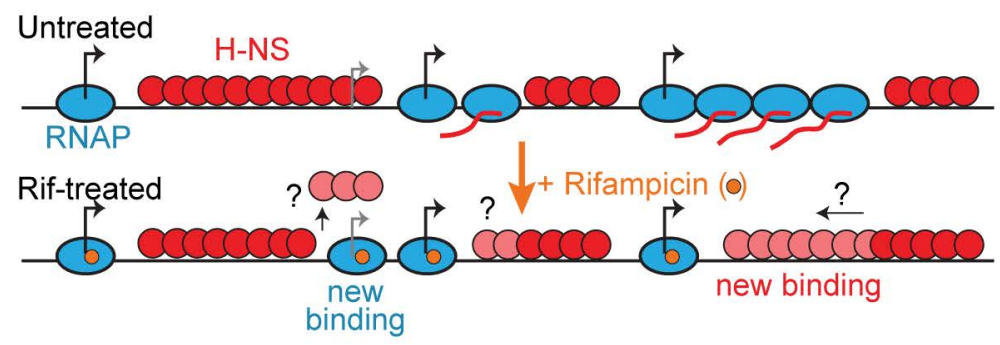

C
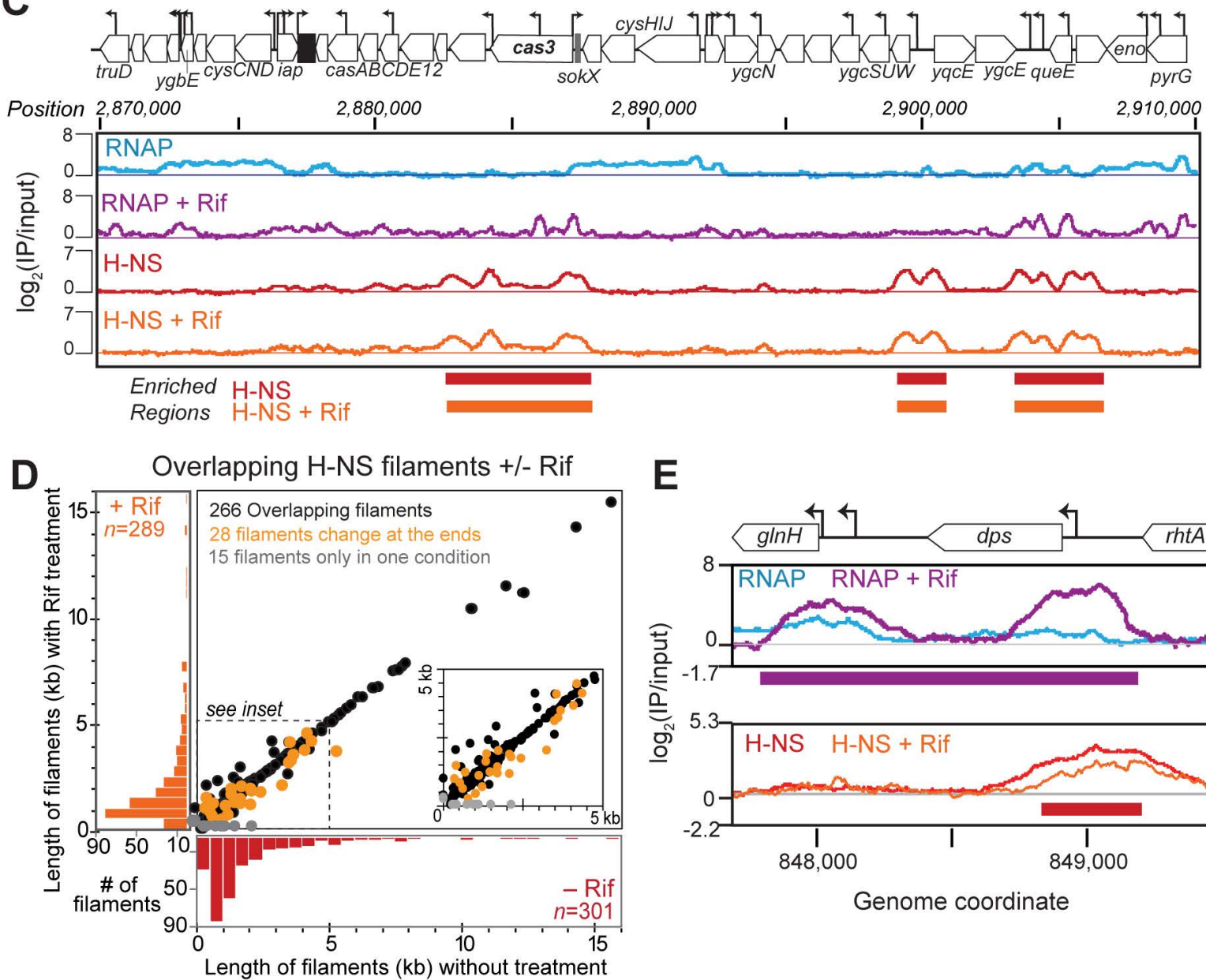

$E$

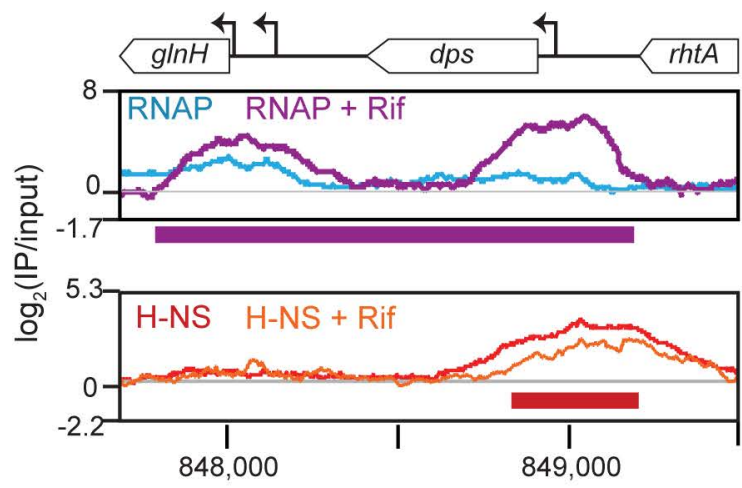

Genome coordinate

Figure 1. Halting transcription does not dramatically change H-NS distribution in vivo. contacting one or two DNA duplexes, respectively. Alternating monomers in the head-head, tail-tail H-NS oligomer are pink and red. In the proposed hemi-sequestration model of linearbridged switching, half the DBDs contact one DNA duplex and the other half are sequestered by interaction with the NTD in a linear filament. To switch to a bridged filament, the sequestered DBDs are released and contact the second DNA duplex. (B) Schematic of ChIP-seq experiment. If H-NS (red) filament boundaries are determined principally by actively transcribing RNAP (blue), then treatment with rifampicin (Rif, orange), which traps RNAP at promoters, should change the H-NS binding pattern. (C) ChIP-seq locations of RNAP in the absence (blue) or presence (purple) of Rif and of H-NS in the absence (red) or presence (orange) of Rif.

1117 Representative ChIP-seq reads were normalized ratios to the IP input DNA reads and are shown 
1118 as $\log 2$ ratios $(n=2)$ with baselines of signal in transcriptionally silenced DNA (RNAP) or

1119 unoccupied regions (H-NS). H-NS enriched regions (i.e., filaments) identified by MOSAiCS are

1120 shown as boxes below ChIP traces. Genes (white boxes) are shown above the coverage tracks.

1121 TSSs (promoters) are indicated by black arrows (Thomason et al., 2015). (D) Length of H-NS

1122 enriched regions identified by MOSAiCS in Rif-treated (red) or untreated (orange) cells; binned

1123 distributions are shown on each axis. Filaments that changed in H-NS density at the ends are indicated by light orange dots. Filaments only present in one condition are indicated by gray dots. Number of filaments $(n)$ shown in each graph are indicated. (E) H-NS filament at the $d p s$ promoter in untreated cells (red line) is lost after Rif treatment (orange line), likely due to the appearance of RNAP trapped at the $d p s$ promoter (purple line). Purple and red bars indicate enriched regions identified by MOSAiCS.

1129 Associated data: Figure S1-1, Figure S1-2, and Figure S1-3. 
A

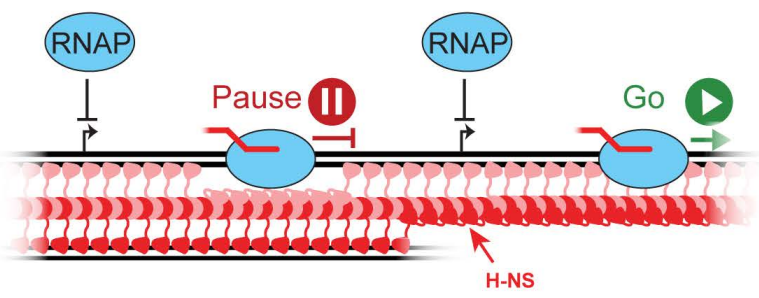

C

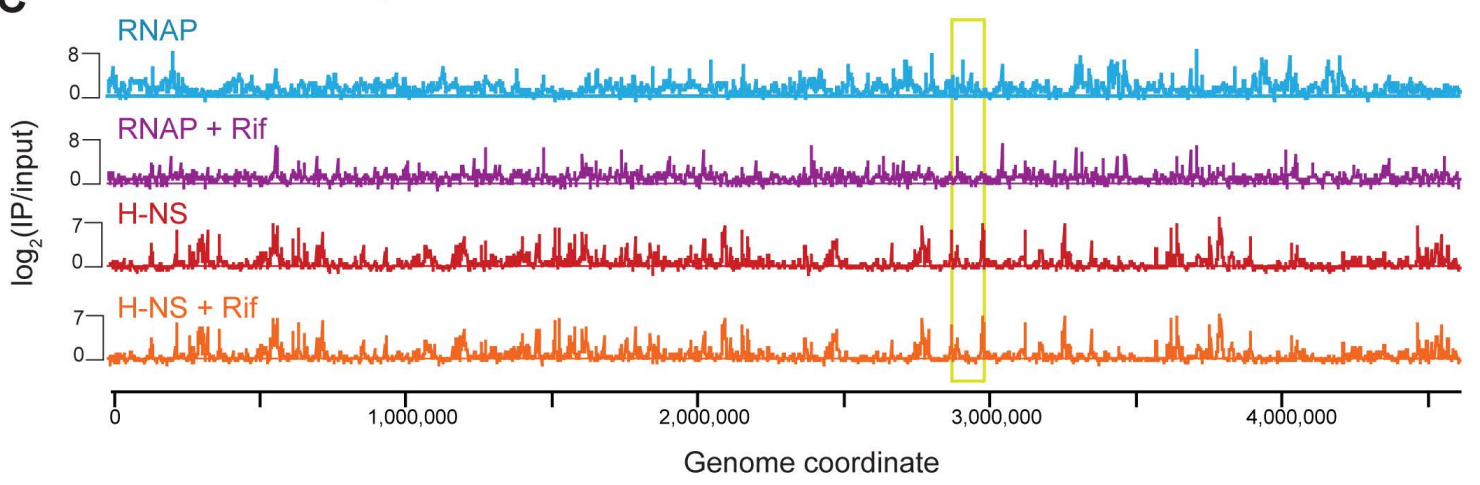

D Comparison to published H-NS ChIP patterns
B

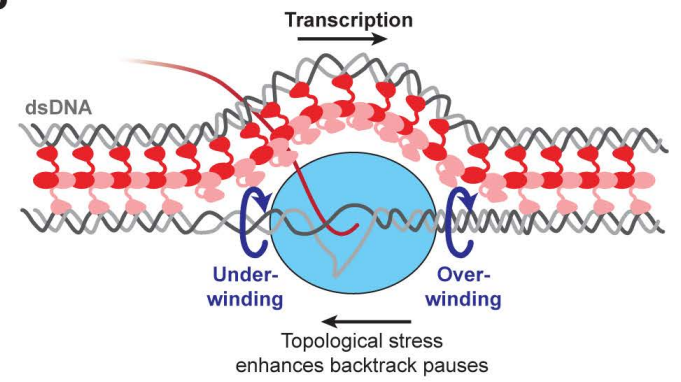

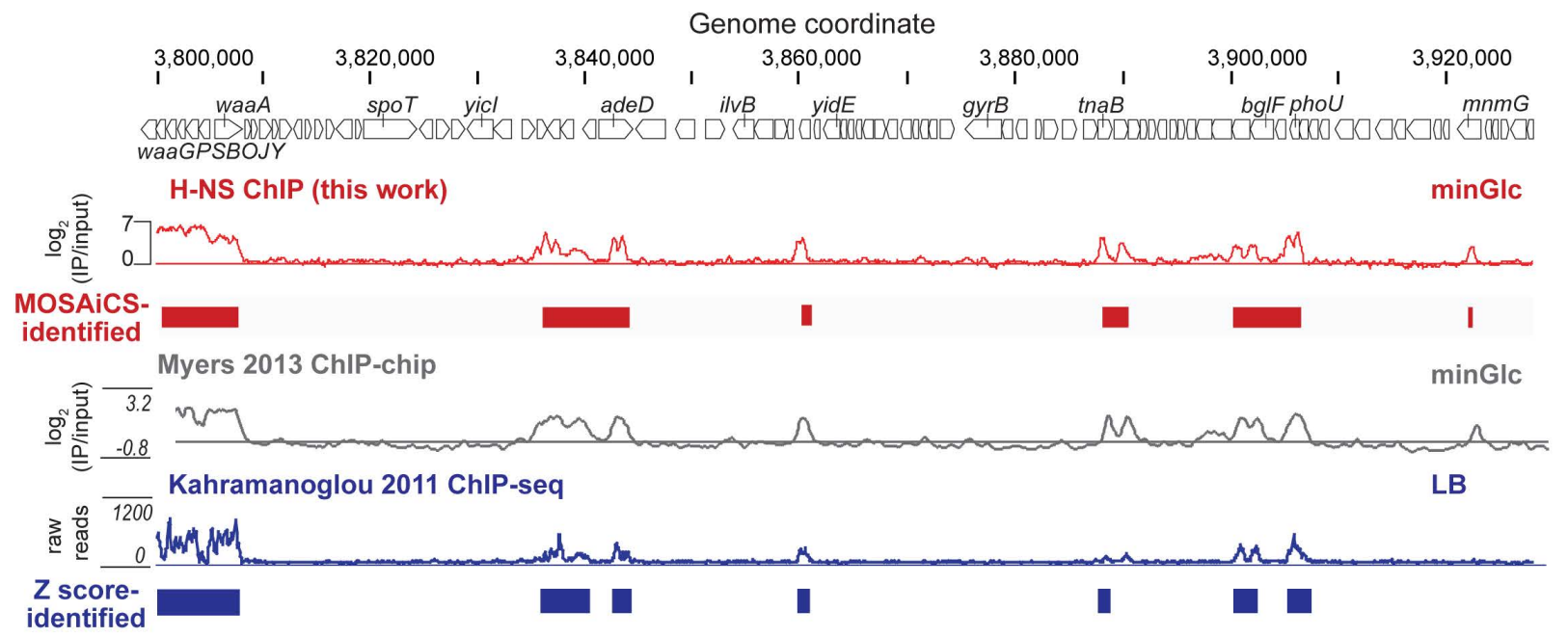

1131 Figure S1-1. H-NS and RNAP ChIP-seq in untreated and Rif-treated cells.

1132 (A) Both bridged and linear H-NS filaments can occlude RNAP from promoters, but only bridged filaments can stimulate pausing by an elongating RNAP. (B) Bridged filaments stimulate pausing by RNAP by creating a closed topological domain in which the DNA is overwound in front of RNAP and underwound behind RNAP. This torsional stress favors backtracking by RNAP and thus enhances backtrack pausing. (C) Normalized read coverage $\left(\log _{2}(\mathrm{IP} /\right.$ input)) of the entire genome for H-NS and RNAP (Beta) IP in the presence (+Rif) or absence of rifampicin. Beta and H-NS ChIP were normalized separately, and the scales are indicated. Yellow box indicates the region shown in Figure 1B. (D) Comparison of H-NS filaments identified in this work by MOSAiCS (Figure 1) to the H-NS binding patterns reported by (Myers et al., 2013) using ChIP-chip profiling of E. coli K-12 MG1655 grown in minimal glucose medium (gray) and the H-NS filaments identified by (Kahramanoglou et al., 2011) using Z-score analysis of ChIP-seq data from E. coli K-12 MG1655 grown in rich medium (Luria broth- $0.5 \% \mathrm{NaCl}$ ) (blue). The H-NS binding patterns are nearly identical in all three conditions. 
bioRxiv preprint doi: https://doi.org/10.1101/2020.06.11.146589; this version posted January 19, 2021. The copyright holder for this preprint (which was not certified by peer review) is the author/funder, who has granted bioRxiv a license to display the preprint in perpetuity. It is made available under aCC-BY-NC-ND 4.0 International license.

\section{5}




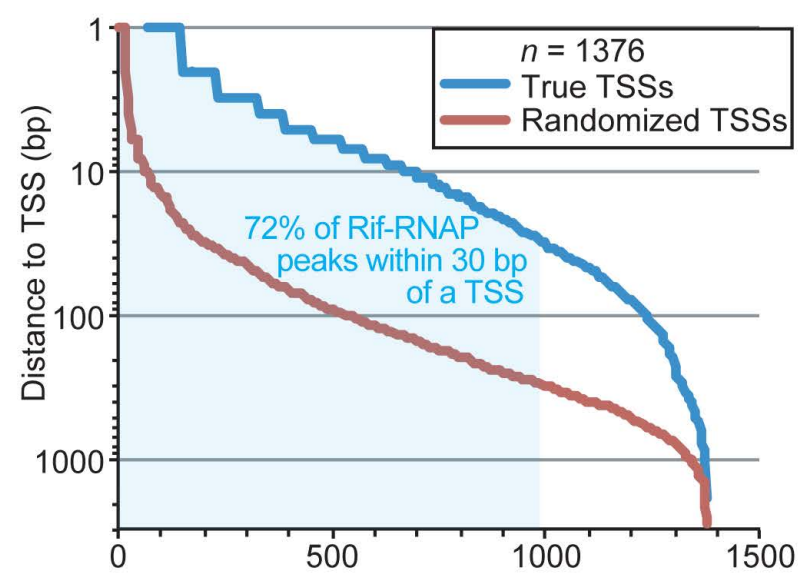

Number of Rif-induced RNAP Peaks

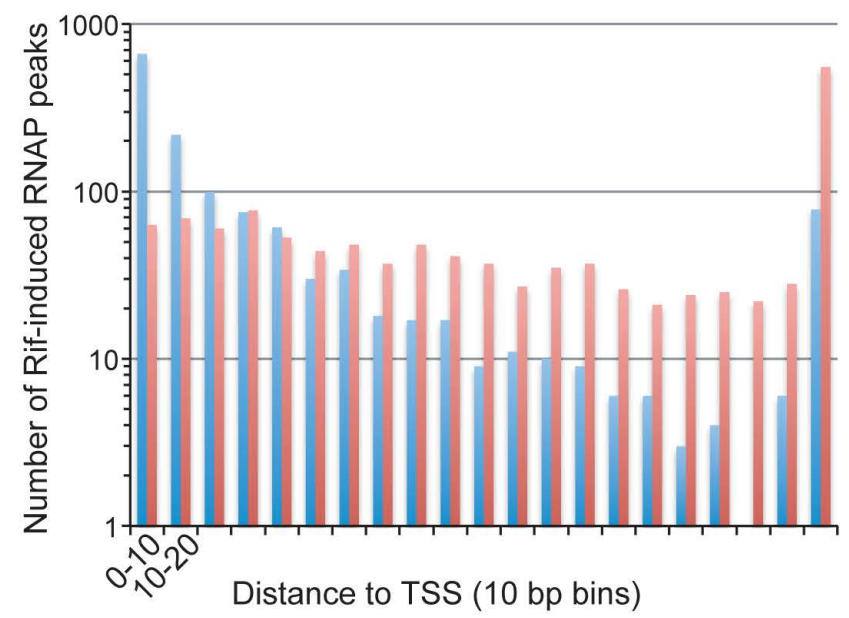

True TSSs $10 \pm 124 \quad p<0.0001$ (Wilcoxon Mann-Whitney U-test)

Randomized TSSs $144 \pm 286$

1147 Figure S1-2. RNAP relocates to known TSS upon Rif treatment.

1148 Comparison of 1376 Rif-induced RNAP peaks to 11944 TSSs reported by (Thomason et al., 2015) for E. coli K-12 MG1655 growing in exponential phase in M9 glucose minimal medium.

1151

1152 Distances from Rif-induced RNAP peaks to the nearest TSS were compared to the same calculation for randomized TSSs $\left(\right.$ E. coli genome rotated $\left.90^{\circ}\right)$. 


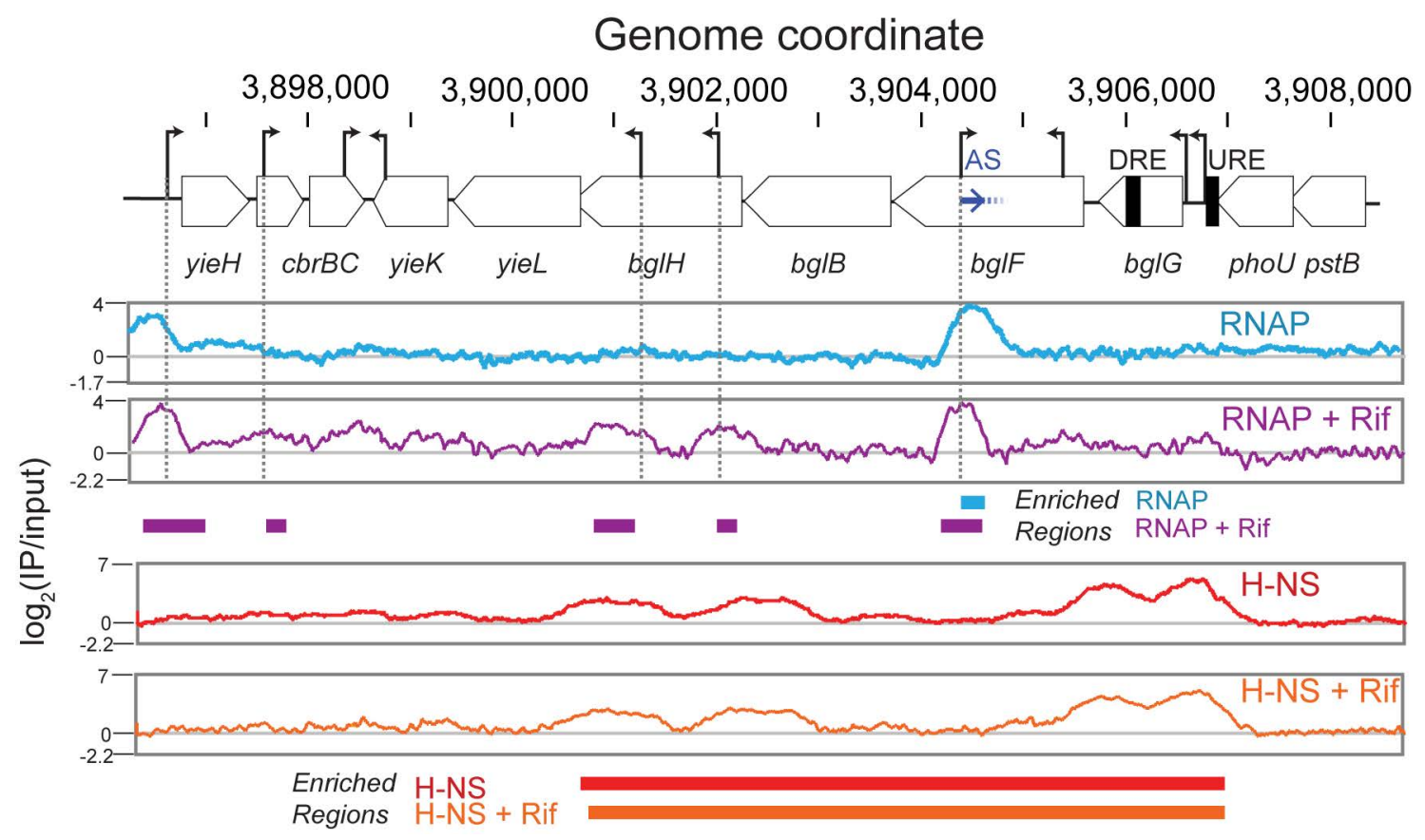

Figure S1-3. Elimination of transcript elongation in the silenced $b g l$ operon by Rif treatment does not change the H-NS binding pattern. RNAP and H-NS ChIP-seq maps of the H-NS-silenced $b g l$ operon in the presence or absence of Rif. Traces are representative of two replicates. The high-affinity H-NS binding sequences (downstream regulatory element, DRE, and upstream regulatory element, URE) are shown as black boxes. The $b g l F$ antisense transcription unit is shown as a blue arrow (AS). Enriched regions identified by MOSAiCs are indicated by solid bars below the plot (note that MOSAiCs did not identify the full length of the $b g l F$ antisense transcription unit). Other colors as in Figure 1. Genes (white boxes) are shown above the coverage tracks. TSS identified in (Thomason et al., 2015) are indicated by black arrows. 
A

In vitro Nitro-seq assay

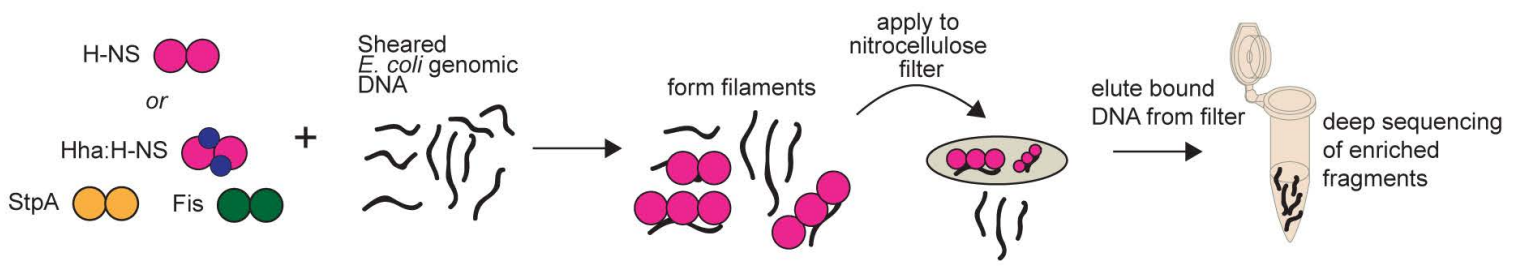

\section{B}

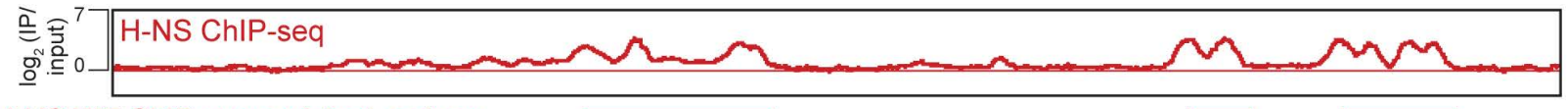

H-NS WT ChIP-seq enriched regions

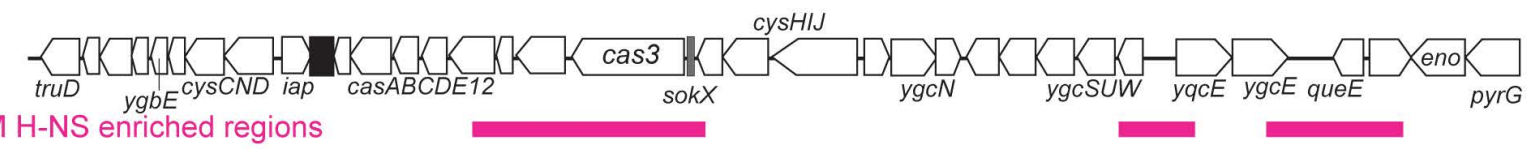

$100 \mathrm{nM} \mathrm{H}-\mathrm{NS}$ enriched regions

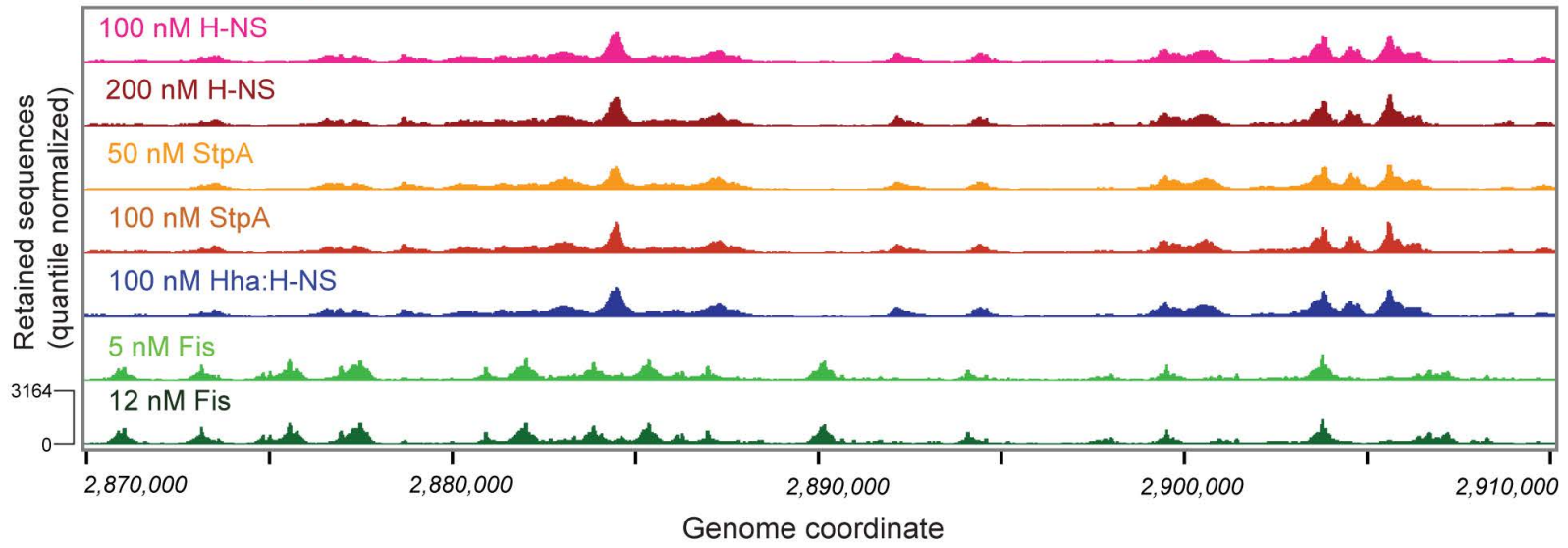

Figure 2. H-NS filaments can be reconstituted in vitro.

(A) The Nitro-seq assay to determine sequence binding preferences, illustrated here for H-NS, StpA, Hha:H-NS, or Fis. Proteins are mixed with sheared genomic DNA and then applied to a nitrocellulose filter (Figure S2-1,2). Two concentrations of H-NS, StpA, and Fis were compared to ensure features identified were not highly concentration-dependent (see Figure S2-1,2). Retained DNA fragments are eluted and then subjected to HT DNA sequencing (see Methods). (B) Nitro-seq reads in a $40 \mathrm{~kb}$ region of the E. coli K-12 genome for H-NS (pink and dark red), StpA (yellow and orange), Hha:H-NS (blue) and Fis (light and dark green). Reads were quantile normalized to aid comparison between samples. Traces are representative coverage of three replicates. Normalized H-NS ChIP-seq coverage (Figure 1) is shown above the Nitro-seq data for comparison (bright red). Genes in this region are indicated by white boxes. CRISPR repeats are indicated by a black rectangle. The H-NS enriched regions (i.e., filaments) identified by MOSAiCS from the Nitro-seq (100 nM H-NS) and ChIP-seq reads are shown by colored bars. Associated data: Figure S2-1, Figure S2-2, Figure S2-3, and Figure S2-4. 
A

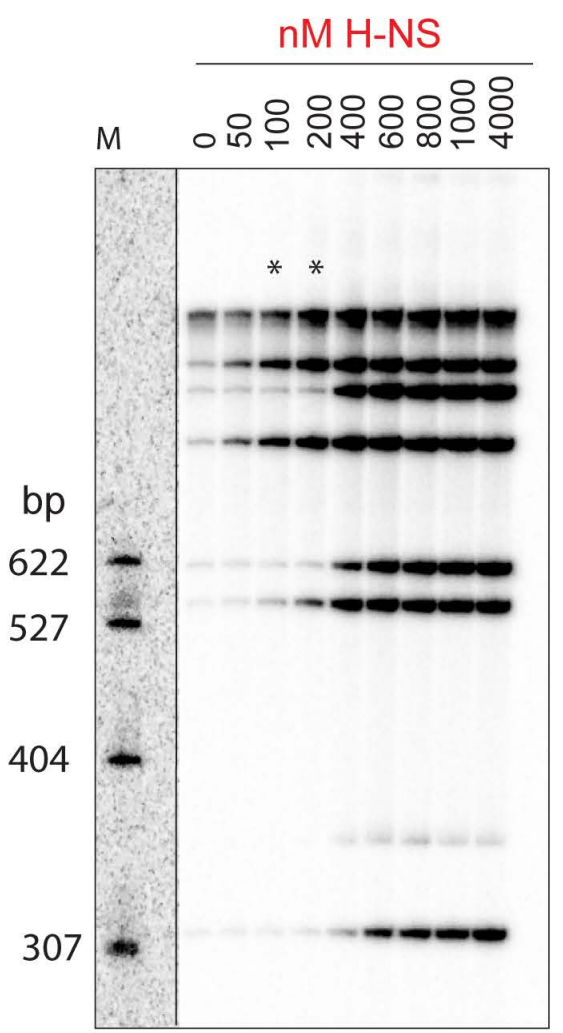

\%counts eluted

from filter:

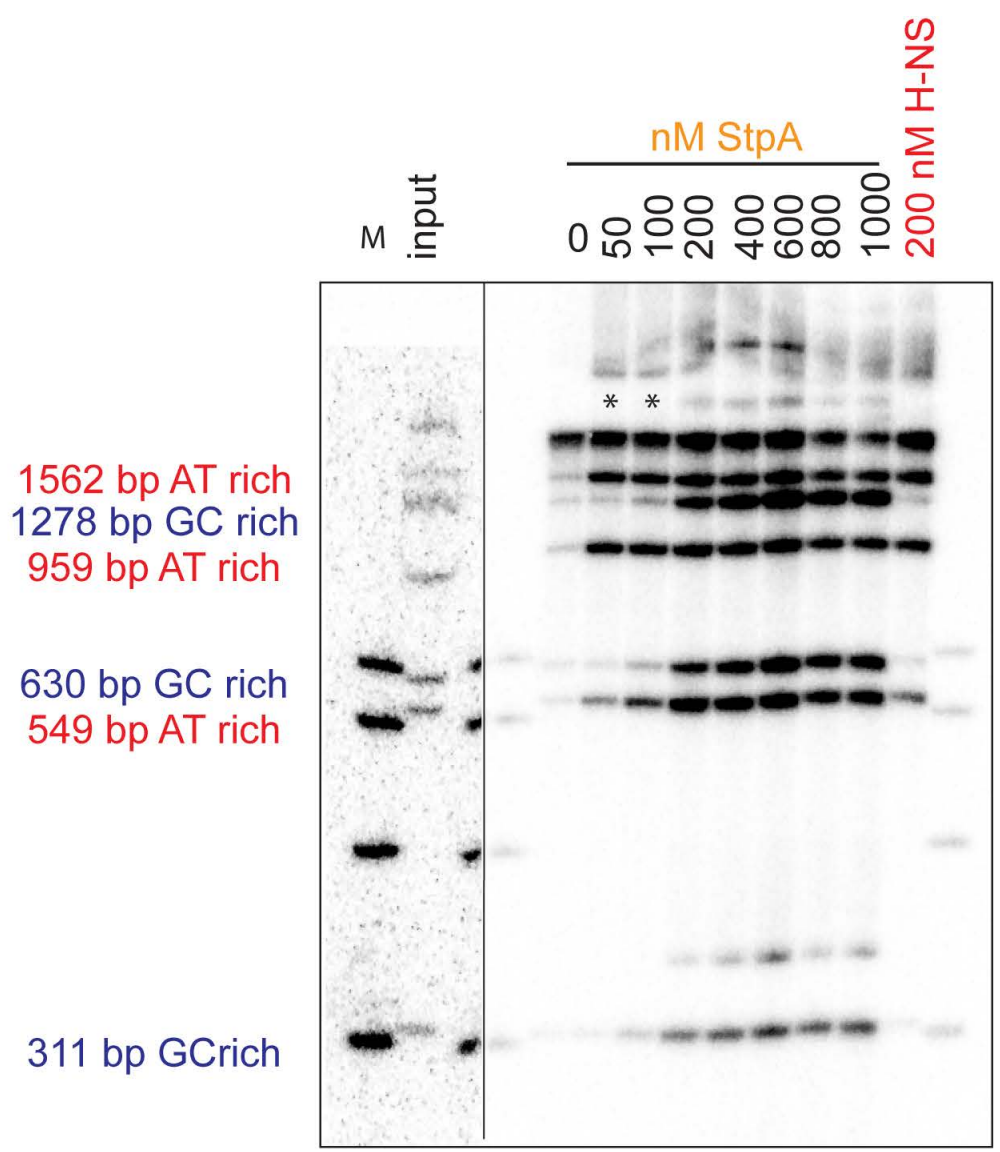

B

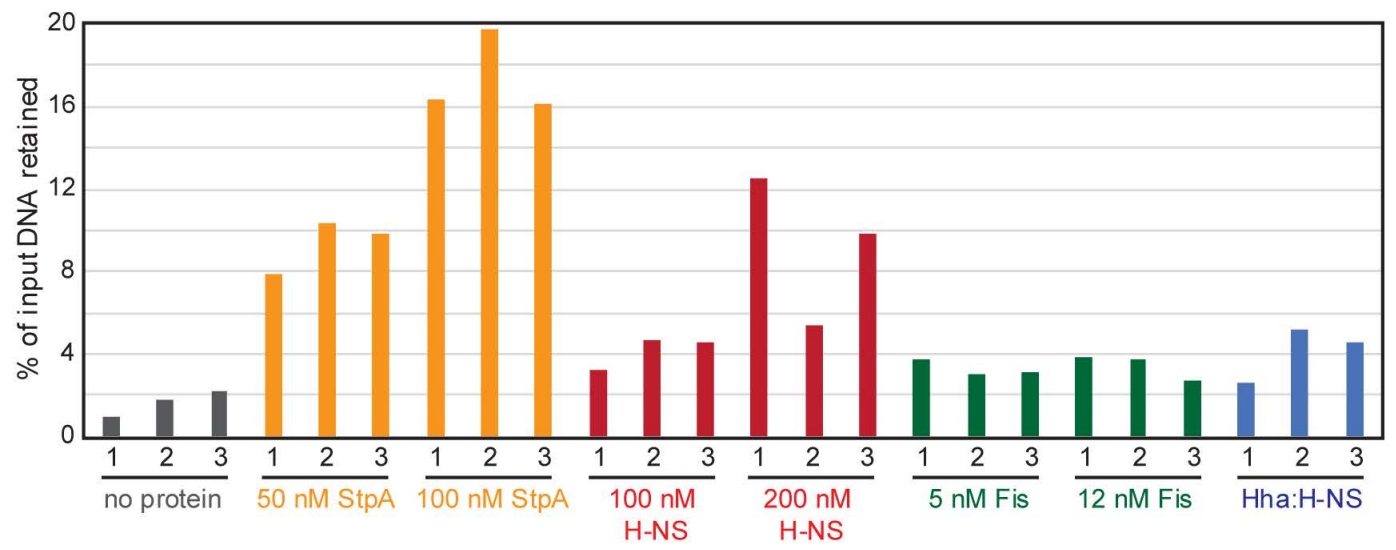

Figure S2-1. Identification of selective conditions for H-NS and StpA Nitro-seq. DNA from pBR322 (blue labels) were mixed with sheared gDNA and either H-NS or StpA at the indicated concentrations and then incubated at $20{ }^{\circ} \mathrm{C}$ for $20 \mathrm{~min}$. The samples were applied to nitrocellulose filters, washed, eluted, ethanol precipitated and then separated by $6 \%$ denaturing PAGE. PCR fragment sizes are shown between the gel panels colored to indicate GC(blue)- vs $\mathrm{AT}(\mathrm{red})$ - richness (Table S3). We predicted that H-NS and StpA would bind to AT-rich, but not GC-rich fragments (Bouffartigues et al., 2007; Dole et al., 2004). Asterisks indicate concentrations selected for Nitro-seq analyses. M indicates ${ }^{32} \mathrm{P}$-labeled MspI digested pBR322 used as size ladder. The marker and input portions of the gels had lower signals than the samples, 
bioRxiv preprint doi: https://doi.org/10.1101/2020.06.11.146589; this version posted January 19,2021. The copyright holder for this preprint (which was not certified by peer review) is the author/funder, who has granted bioRxiv a license to display the preprint in perpetuity. It is made available under aCC-BY-NC-ND 4.0 International license.

1190 so the signal intensity was increased for clarity in these regions (boxed regions). (B) Fraction of 1191 sheared gDNA retained in each replicate of the nitrocellulose binding experiment. ${ }^{32} \mathrm{P}$ counts 1192 before and after elution were measured by liquid scintillation counting. 


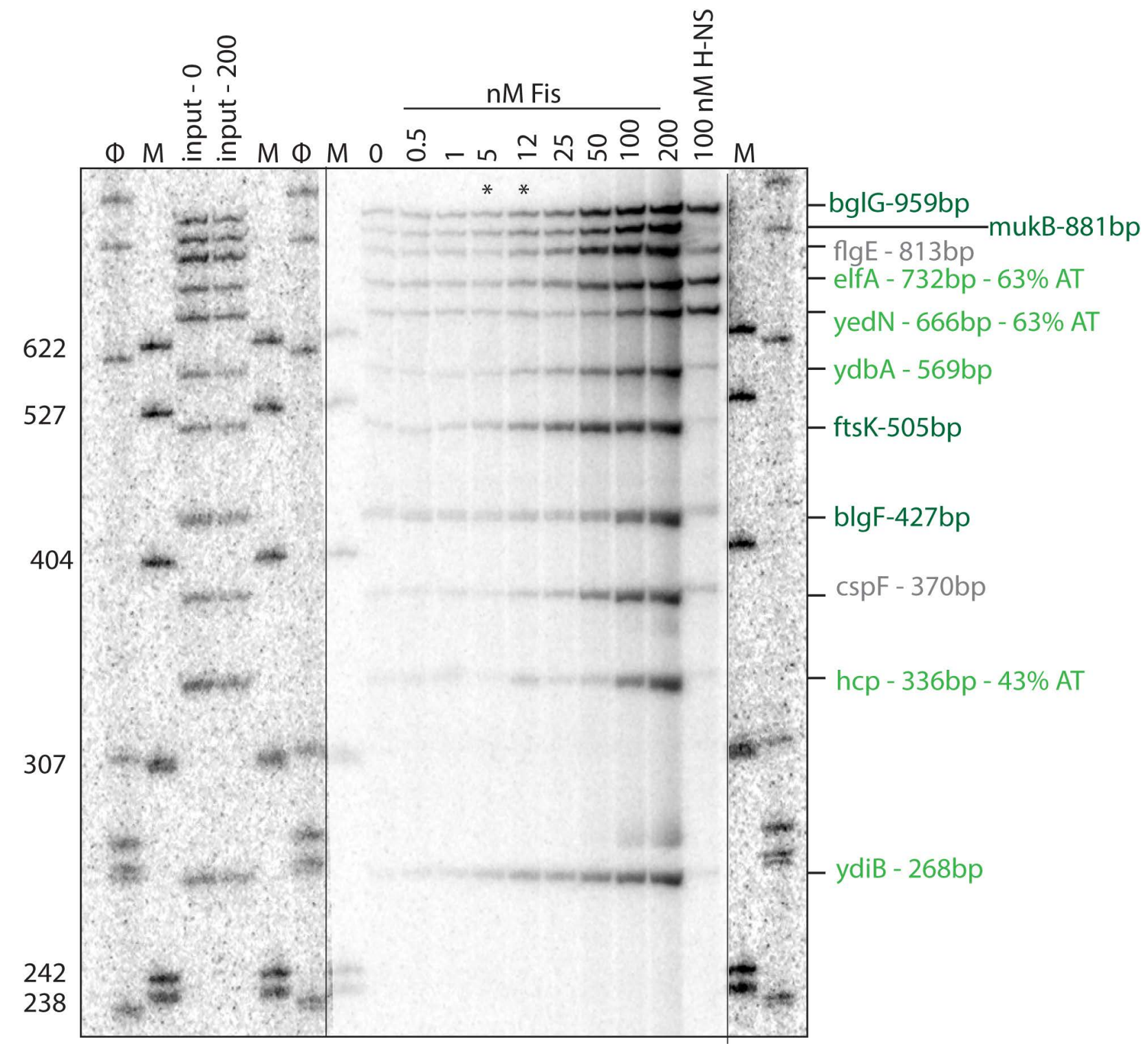

Figure S2-2. Determining selectivity of Fis in nitrocellulose binding assay. To optimize conditions for Fis binding in the Nitro-seq assay, ${ }^{32} \mathrm{P}$-labeled DNA fragments prepared by PCR of purified E. coli genomic DNA were mixed with sheared, deproteinized gDNA and Fis at the indicated concentrations and then incubated at $20^{\circ} \mathrm{C}$ for $20 \mathrm{~min}$. Samples were applied to nitrocellulose filters, washed, eluted, ethanol precipitated, and then separated by $6 \%$ denaturing PAGE. Fragment sizes are indicated on the right colored to indicate predicted Fis binding (no binding, gray; some Fis binding, light green; and strong Fis binding, dark green) (Table S3). As expected, the $f l g E$ and $c s p F$ fragments, which lack predicted Fis sites, were not retained on the filter, whereas other fragments like $f t s K$, which contains predicted Fis sites, were retained. Fis concentrations of 5 and $12 \mathrm{nM}$ (asterisks) were selected for Nitro-seq. M, ${ }^{32} \mathrm{P}$ labeled MspI digested pBR322. $\Phi,{ }^{32} \mathrm{P}-$-labeled HaeIII digest of $\Phi$ X174 DNA. The signal intensities for the boxed left and right sides of the gel were relative to the samples for clarity. 

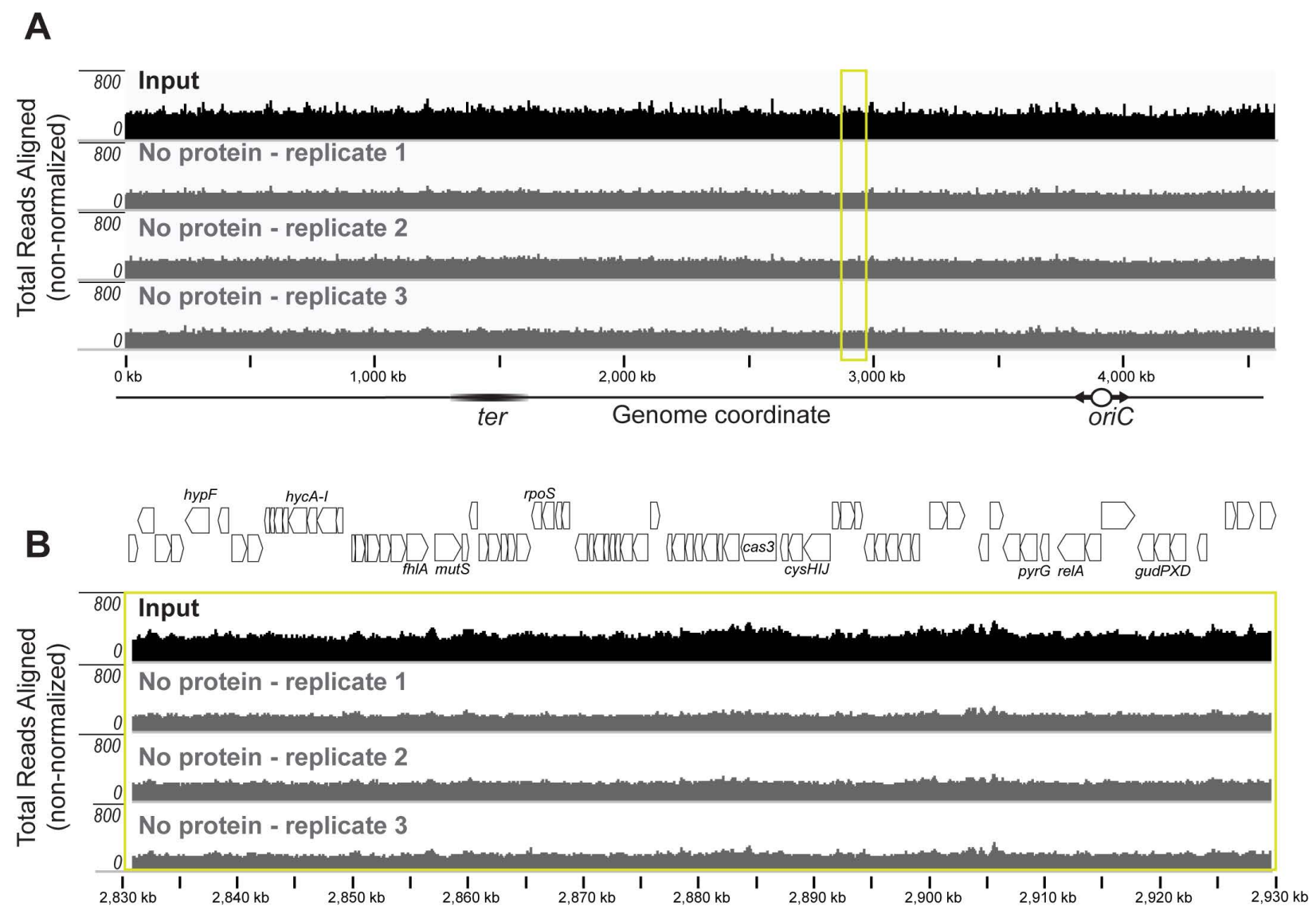

\section{Figure S2-3. gDNA for Nitro-seq was a uniform across genome and free of protein contamination.}

(A) DNA-seq read (unnormalized) for the input (black) and no protein samples (replicates 1-3; gray). (B) Expanded view of the yellow box in (A) showing even coverage in each input sample. Genes are shown by white boxes with a subset of names indicated. The no protein samples correspond to gDNA retained on the nitrocellulose filter. If residual protein was present in the sheared gDNA, peaks of enrichment would present in the no protein samples (we observed such peaks when gDNA was incompletely purified). 
A

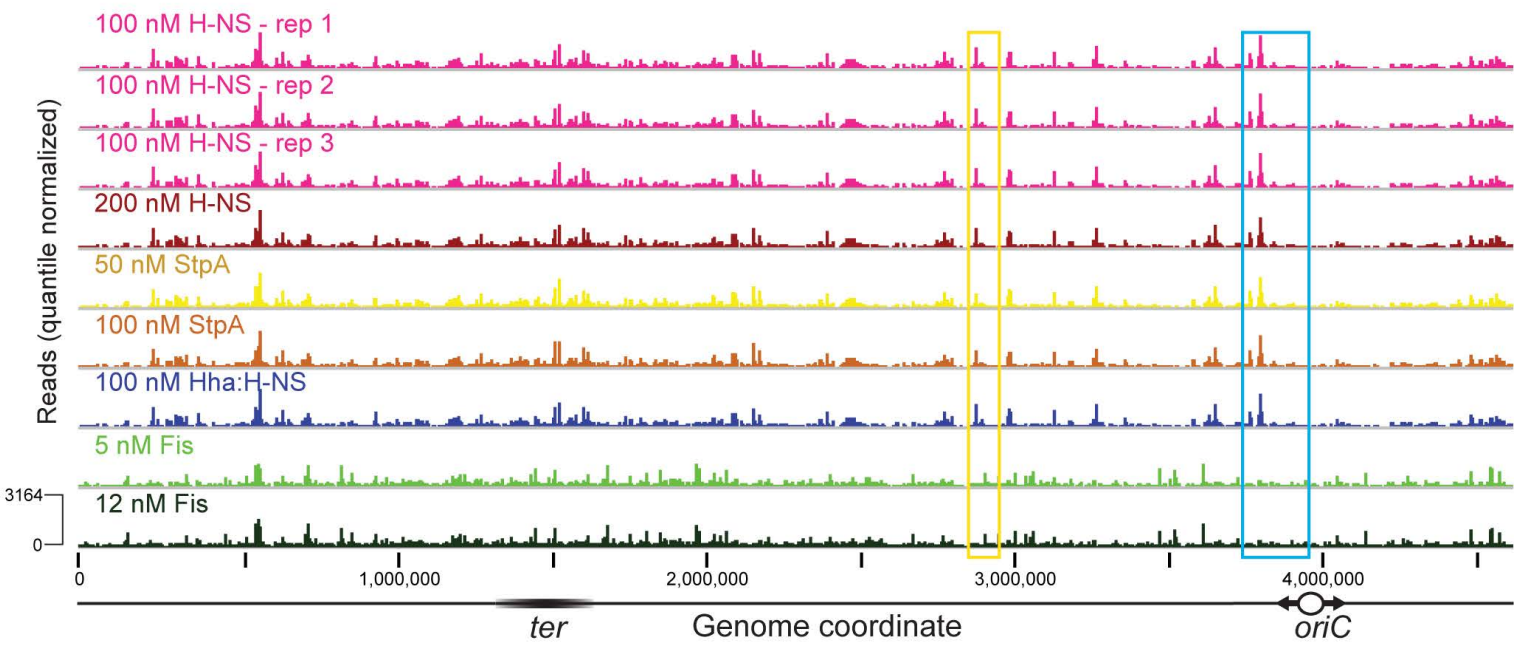

B

\section{Genome coordinate}

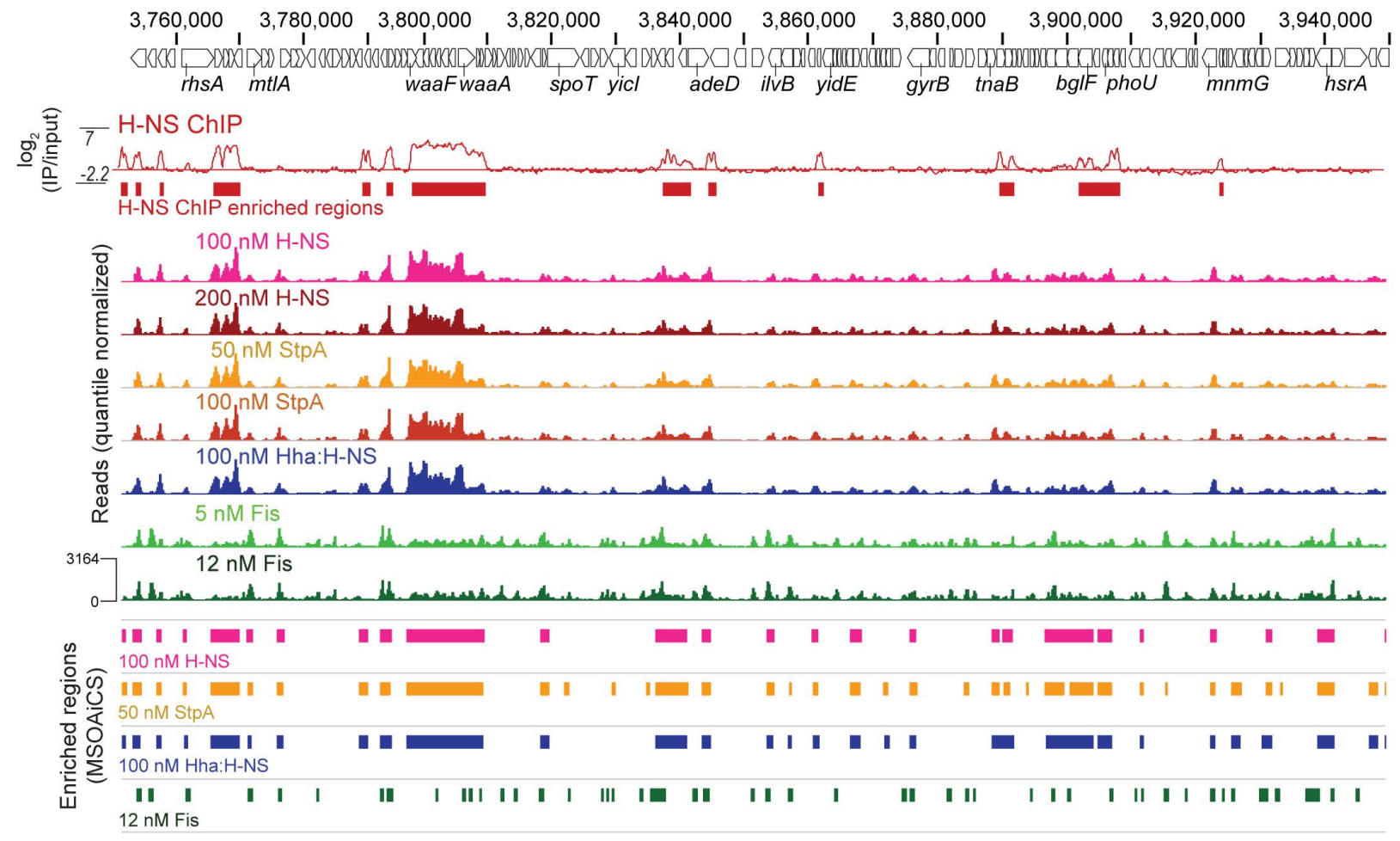

1218 Figure S2-4. Binding of H-NS, StpA, Hha:H-NS, and Fis to genomic DNA in vitro.

(A) Genome-wide views of Nitro-seq results. Reads for Nitro-seq were quantile normalized to each other consistency, but not to ChIP-seq coverage. The yellow box indicates the region shown in Figure $2 \boldsymbol{B}$. The cyan box indicates the region shown in panel B of this figure. (B) View of Nitro-seq and H-NS ChIP-seq results in a $197 \mathrm{~kb}$ region of the genome. Traces are representative of triplicate samples. MOSAiCS enriched regions found in at least 2 of 3 replicates for each condition are shown by colored boxes below coverage plots. 
A

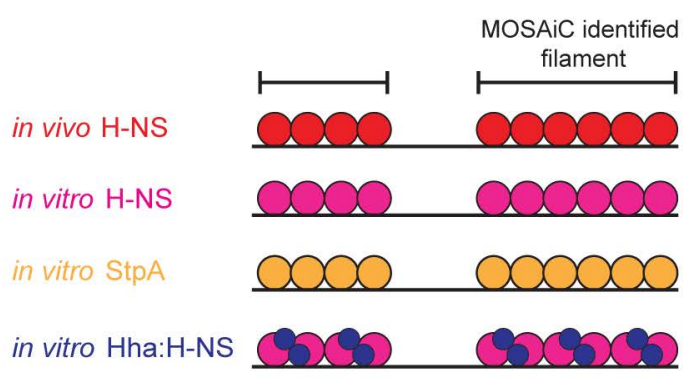

genome coordinate

C

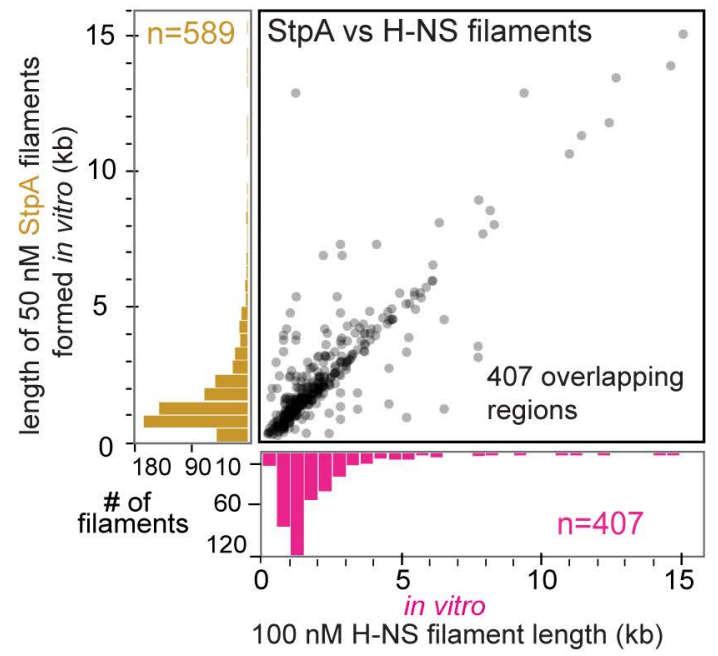

B

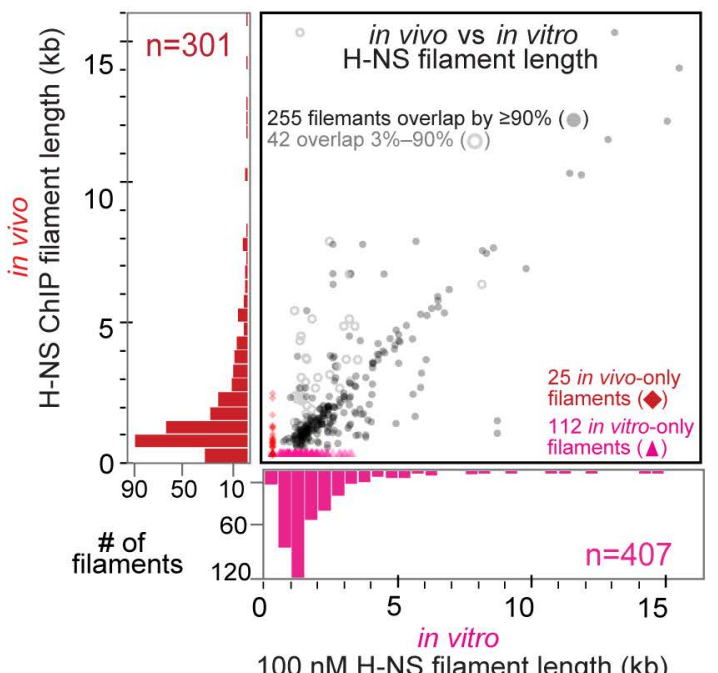

D

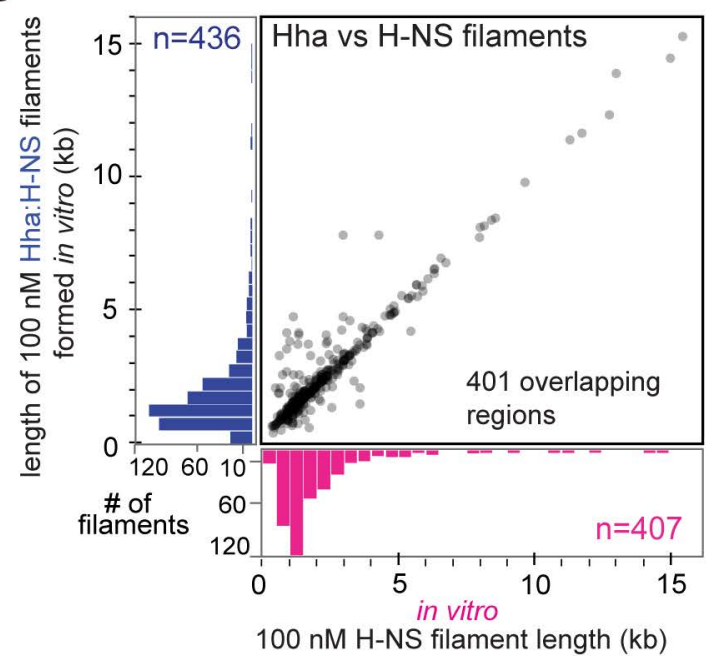

Figure 3. Nitro-seq revealed similar sequence specificity for H-NS in vivo, H-NS in vitro, StpA in vitro, and Hha:H-NS in vitro.

(A) Color code for comparisons of the filament lengths determined by MOSAiCs for H-NS, StpA, and H-NS:Hha. (B-D) Scatter plots of the lengths of filaments determined by MOSAiCS. The distributions of filaments lengths are shown as histograms on each axis. Lengths of in vitro $100 \mathrm{nM}$ H-NS filaments are on each $\mathrm{x}$-axis and the $\mathrm{y}$-axis shows filament lengths for (B) in vivo H-NS, (C) $50 \mathrm{nM} \mathrm{StpA}$, and (D) $100 \mathrm{nM}$ Hha:H-NS filaments. The number of filaments $(n)$ is indicated for each sample. The numbers of filaments overlapping with the in vitro H-NS filaments by at least $1 \mathrm{bp}$ is indicated in each panel. (B) 297 filaments overlapped between in vitro and in vivo H-NS. Only 255 overlapped by $90 \%$ (i.e., the percent of the length of the Nitroseq filament that overlaps with the ChIP-seq filament and vice versa) and 42 overlapped by $<90 \%$ of the total length (gray outlined circles). For the 255 overlapping points, transparent black circles where plotted so that darker colors indicate more points on the graph. Filaments only found in vitro (112, pink triangles) or in vivo (25, red diamonds) are shown along the axes. (C) For each of the 407 overlapping filaments between in vitro StpA and in vitro H-NS, transparent black circles were plotted so that darker colors indicate more points on the graph. (D) Same as in $\mathrm{C}$ but for the 401 overlapping regions between in vitro Hha:H-NS and in vitro H-NS. Associated data: Figure S3-1 and Figure S3-2. 
A
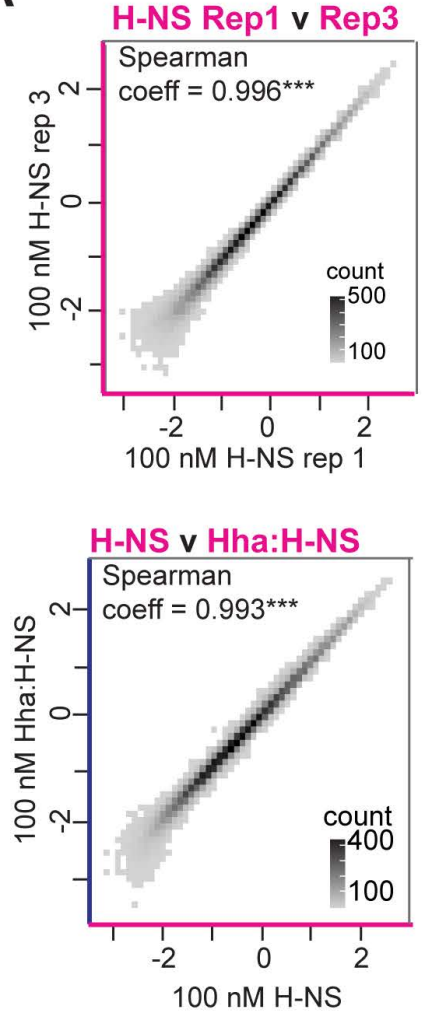
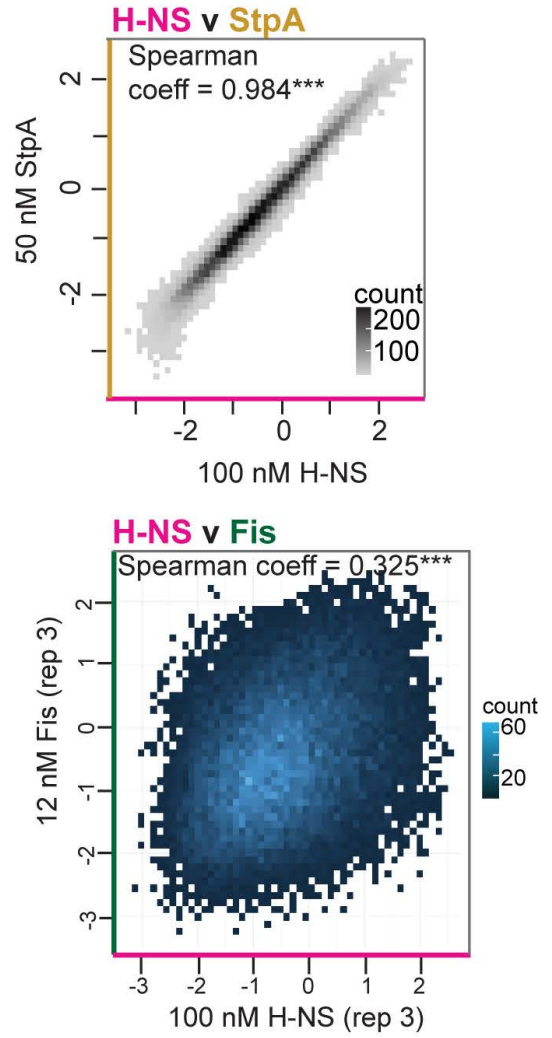

B

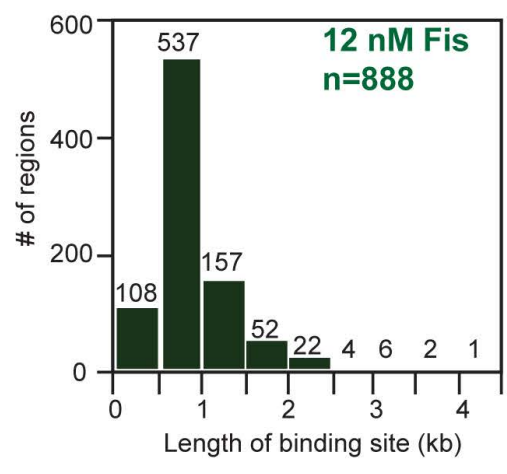

C

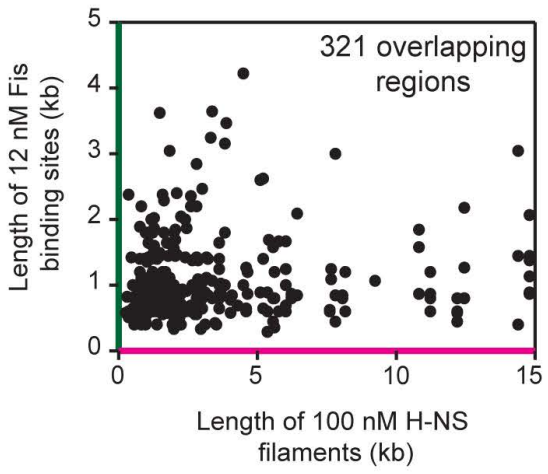

Figure S1-1. DNA binding pattern of $\mathrm{H}-\mathrm{NS}$ in reconstituted filaments is distinct from Fis, but similar to H-NS in vivo, StpA, and Hha:H-NS.

These panels compare read densities binned into $200 \mathrm{bp}$ intervals rather than the MOSAiCsidentified filaments shown in Figure 2 to enable more direct comparison of the similarities and differences in binding patterns. (A) Binned scatter plot comparing average $\log _{2}$ (Nitro-seq/input) in $200 \mathrm{bp}$ bins across the genome for $100 \mathrm{nM} \mathrm{H-NS}$ replicate 1 vs. replicate 2 (pink axis); for 100 nM H-NS vs $50 \mathrm{nM} \mathrm{StpA} \mathrm{(gold} \mathrm{y-axis);} \mathrm{for} 100 \mathrm{nM}$ H-NS vs $100 \mathrm{nM}$ Hha:H-NS (blue y-axis); and for $100 \mathrm{nM} \mathrm{H}-\mathrm{NS}$ vs $12 \mathrm{nM}$ Fis (green y-axis). ${ }^{* * *}$ indicates p-value $<2.2 \times 10^{-16}$ for the Spearman coefficient correlation test. (B) Distribution of lengths of Fis (12 nM) binding regions. The number of regions in each bin is indicated. (C) Comparison of lengths of $100 \mathrm{nM} \mathrm{H}-\mathrm{NS}$ filaments (x-axis) overlapping with $12 \mathrm{nM}$ Fis binding sites (y-axis). The total overlapping binding sites (312) resulted from 251 H-NS filaments overlapping with 321 Fis binding sites, indicating multiple Fis binding sites occur at the same genome coordinate as a single $\mathrm{H}-\mathrm{NS}$ filament. 


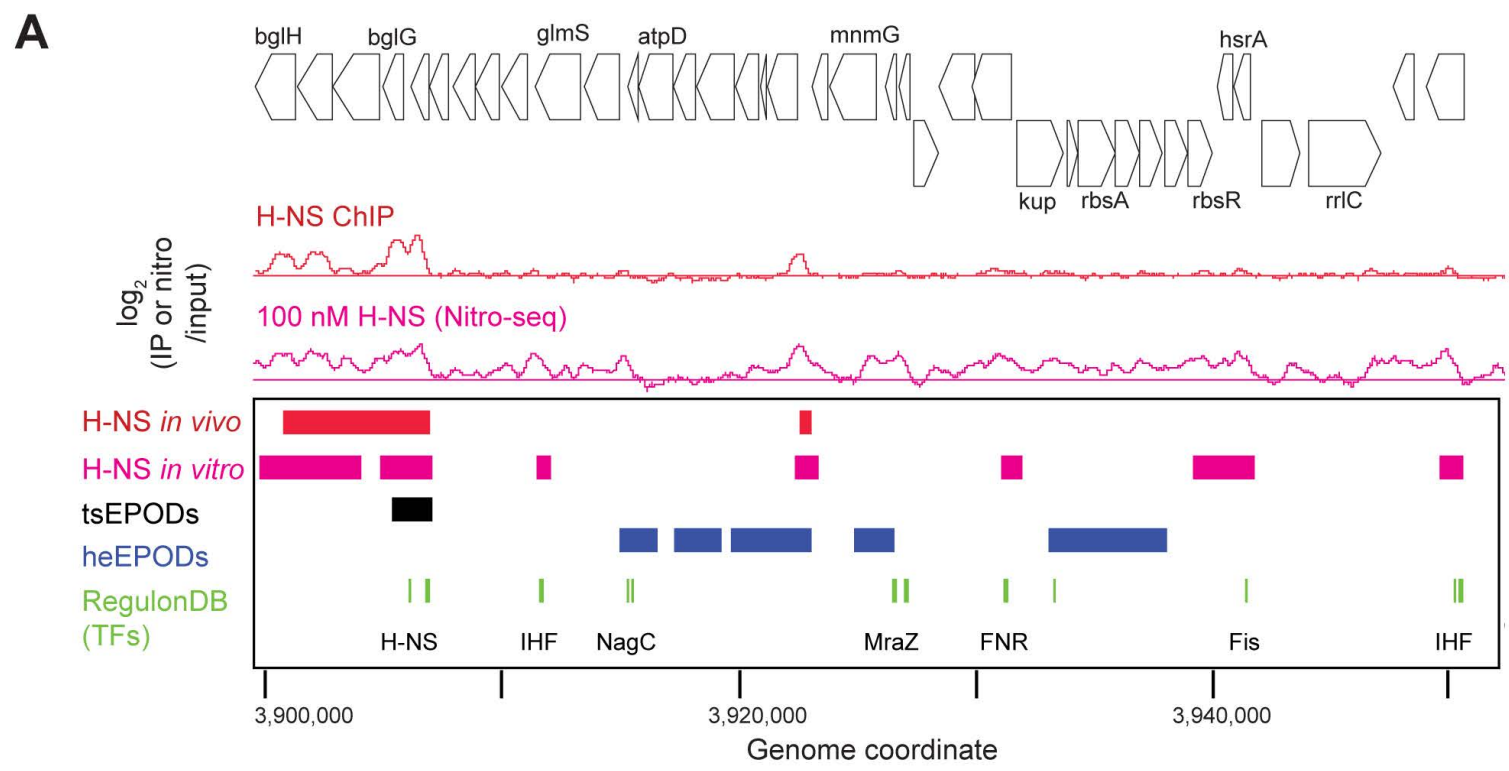

B

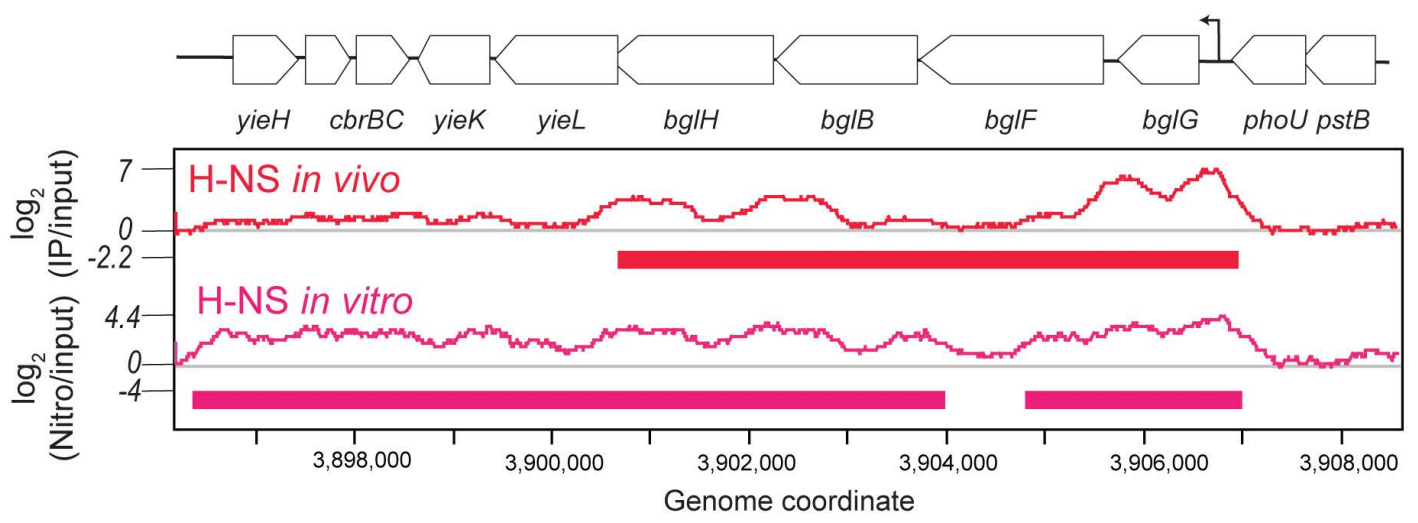

C

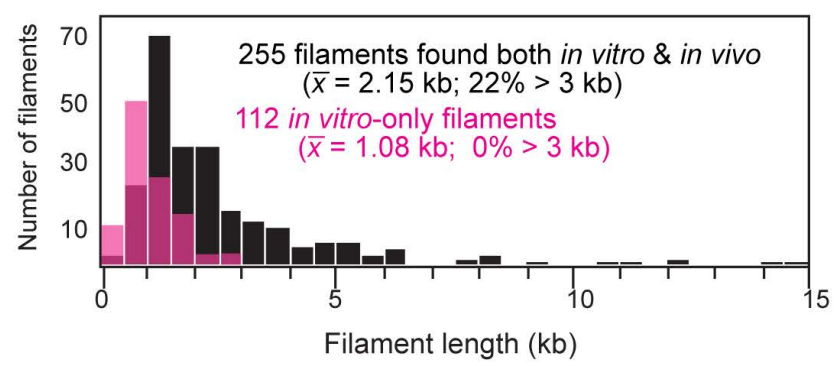

D Overlap between in vitro-only H-NS filaments and known protein binding sites - 112 in vitro-only filaments 112 random sites

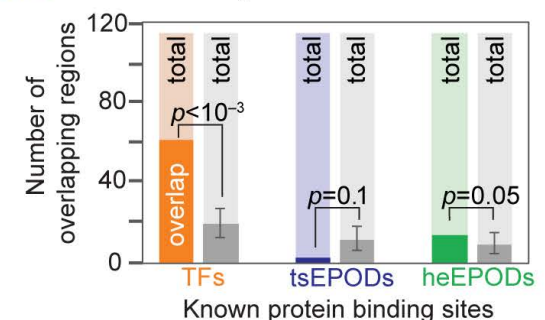
NS binding sites were only present in vitro as illustrated by the read coverage and MOSAICs regions identified in this genomic region. Locations of extended protein occupancy domains (EPODs) that are transcriptionally silent (tsEPODs) or highly expressed (heEPODs) are indicated (black and blue respectively (Vora et al., 2009). TF binding sites as annotated in RegulonDB are also shown (green; Santos-Zavaleta et al., 2019). (B) View of H-NS ChIP-seq and H-NS Nitro-seq normalized read coverage at the $b g l$ operon. MOSAiCS regions indicated by bars. Similar H-NS binding patterns were observed at the $b g l$ operon in vivo (ChIP-seq, red) and 
in vitro (Nitro-seq, pink). (C) Comparison of Nitro-seq-specific (Class 2) and overlapping (Class 1) H-NS binding region length distributions. (D) Number of Nitro-seq-specific (Class 2) filaments that overlap with three types of known protein binding sites: transcription factor binding sites found in RegulonDB (TFs; dark orange), transcriptionally silent extended protein occupancy domains (tsEPODs; dark blue), or highly expressed EPODS (heEPODs; dark green). The total filaments in class 2 (112) is shown in light colors for each comparison. Statistical significance of this overlap was determined by comparing it to the overlap of 112 random sites generated 1000 times and these known protein binding sites (gray bars and p-values). Error bars indicate the $97.5 \%$ quantile of the random overlap values at the top and the $2.5 \%$ quantile of the random overlap values at the bottom. 
A

12 bp dsDNA
H-NS DBD

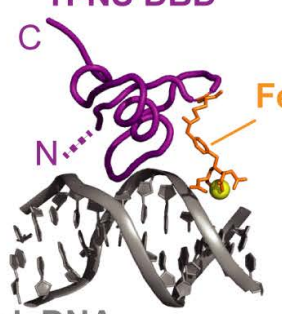

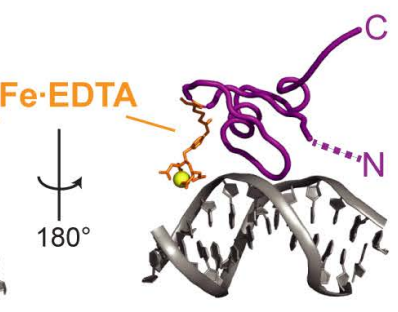

B
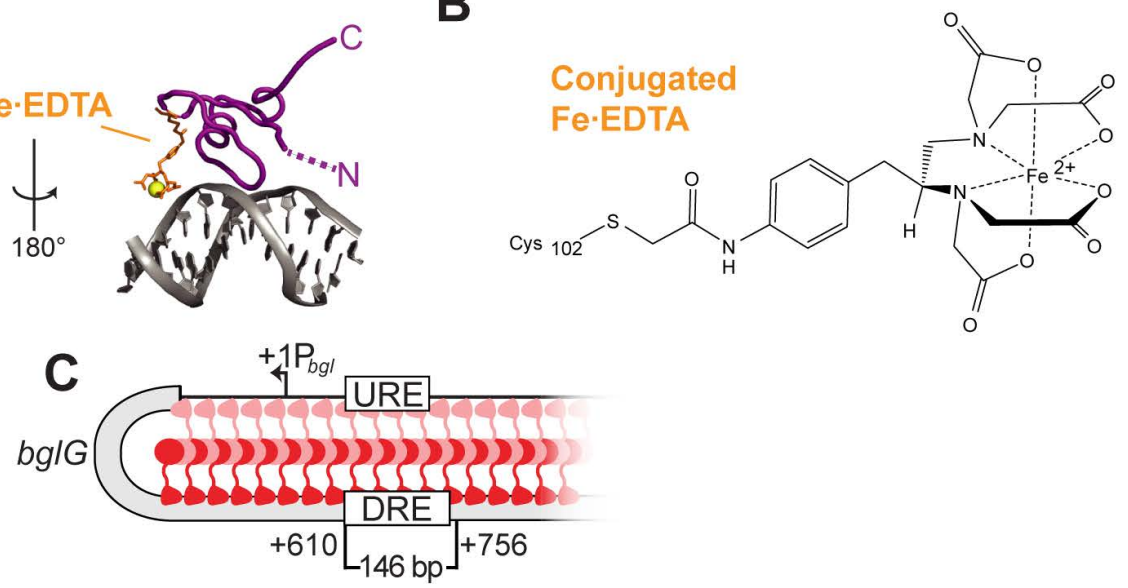

NT Strand

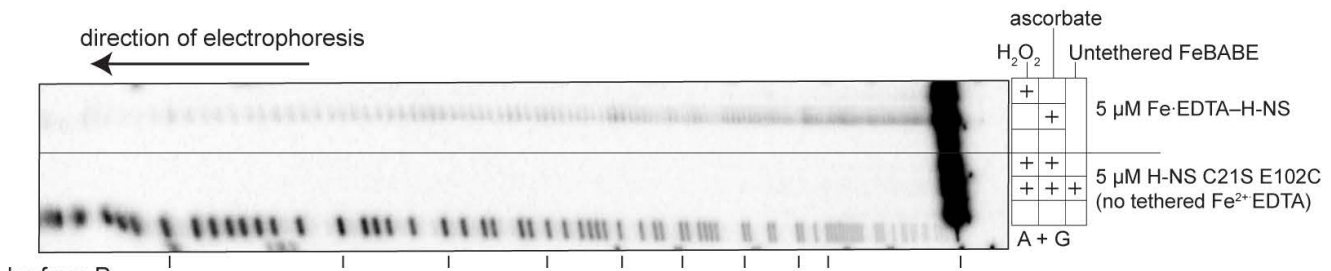

bp from $\mathrm{P}_{b g l} \quad \begin{aligned} & 1 \\ & 637\end{aligned}$ $\begin{array}{llllllll}1 & 1 & 1 & 1 & 1 & 1 & 1 & 1 \\ 649 & 658 & 669 & 678 & 687 & 698 & 709716\end{array}$

uncleaved

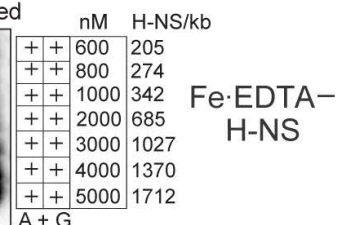

E NT Strand

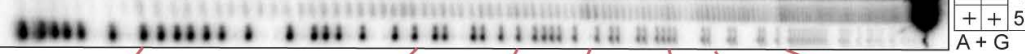

\section{F $T$ Strand}
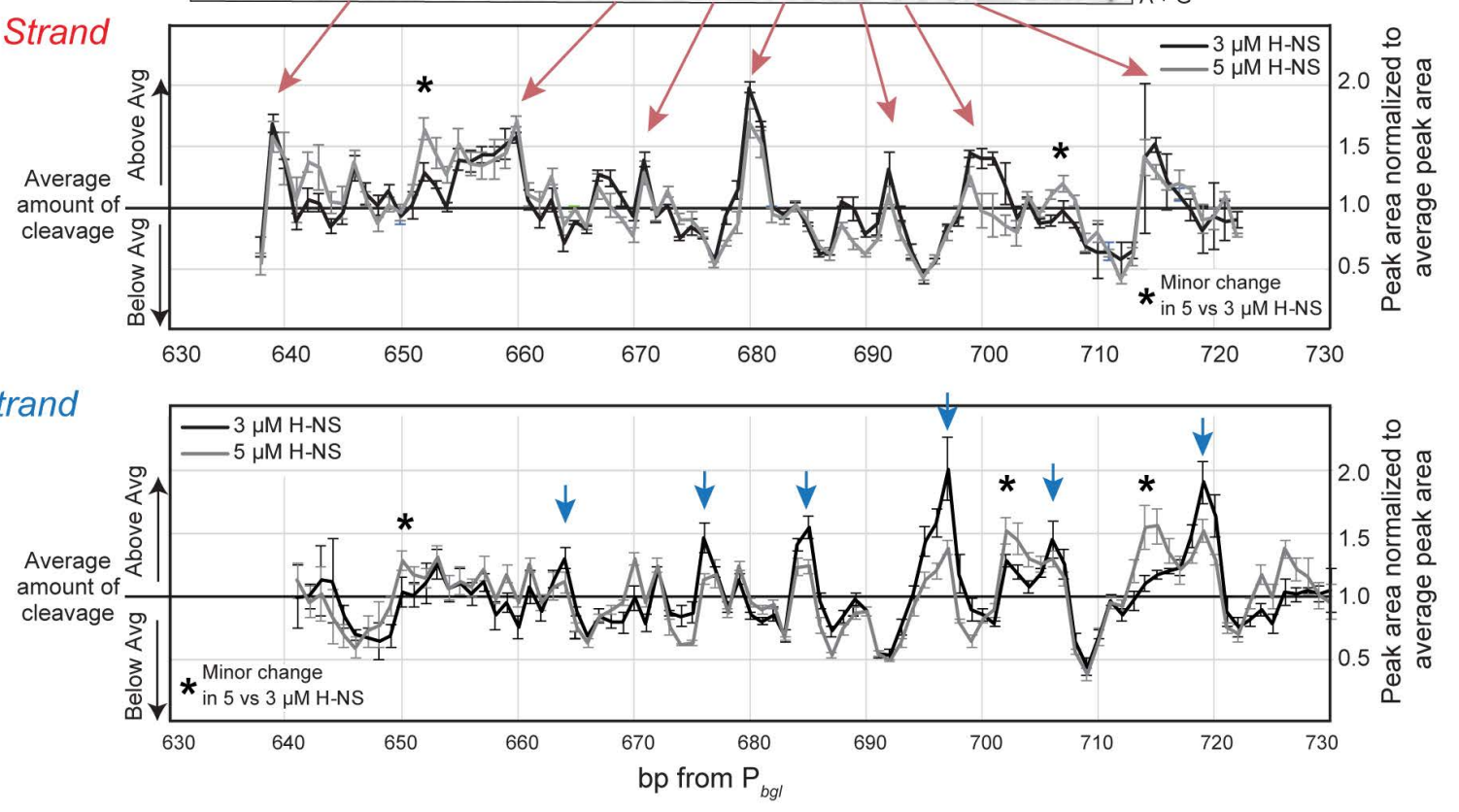

Figure 4. TEN-map cleavage of $b g l$ DRE DNA.

(A) Model of the H-NS DBD (purple; PDB 2L93) bound to 12 bp duplex DNA (gray; (Riccardi et al., 2019) with Fe-EDTA (orange) added at position 102. The linker connecting the DBD to the N-terminal oligomerization domain is shown by the dashed line. (B) Structure of Fe·EDTA conjugated to Cys102. (C) The 146-bp DRE DNA fragment used in the TEN-map assay from the region of the $b g l$ operon 610 to $756 \mathrm{bp}$ downstream of the promoter $\left(\mathrm{P}_{b g l}\right)$, which encodes a 
known high-affinity binding region (DRE; downstream regulatory element; (Dole et al., 2004). H-NS (pink and red monomers as in Figure 1) bound at both the DRE and upstream regulatory element, URE, may form a bridged filament. (D) Denaturing 7\% PAG showing Fe·EDTA-H-NS cleavage pattern of the 146-bp DRE DNA labeled on the NT strand. Increasing concentrations of $\mathrm{Fe} \cdot$ EDTA-H-NS were added to $20 \mathrm{nM}$ DNA to form filaments. Cleavage was initiated with addition of both $2 \mathrm{mM} \mathrm{H} \mathrm{H}_{2}$ and $2 \mathrm{mM}$ ascorbic acid (bottom panel). Little to no cleavage was observed in the presence $5 \mu \mathrm{M}$ Fe.EDTA-H-NS with addition of either $2 \mathrm{mM} \mathrm{H}_{2} \mathrm{O}_{2}, 2 \mathrm{mM}$ ascorbic acid, or water (top). Tethered Fe-EDTA was also required for cleavage as addition of free FeBABE to filaments formed with $5 \mu \mathrm{M}$ H-NS C21S E102C did not result in cleavage of the DNA (middle). A+G indicates Maxam-Gilbert sequencing ladder. (E \& F) Quantification of peak areas using semi-automated footprinting analysis (SAFA) analysis at 3 and $5 \mu \mathrm{M}$ Fe.EDTA-H-NS on the NT (E) or T (F) strands. Peak areas normalized to amount loaded in each lane and then the average peak area in each lane. Peaks areas above 1 indicate cleavage hot spots (arrows) and peak areas less than 1 indicate areas of lesser cleavage. Asterisks indicate areas that showed a slight increase in cleavage in $5 \mu \mathrm{M}$ Fe EDTA-H-NS compared to $3 \mu \mathrm{M} \mathrm{Fe} \cdot \mathrm{EDTA}-\mathrm{H}-$ NS. Error bars show standard deviation of three replicates. Associated data: Figure S4-1, Figure S4-2, Figure S4-3, Figure S4-4, Figure S4-5, Figure S4-6, and Figure S2-7. 
bioRxiv preprint doi: https://doi.org/10.1101/2020.06.11.146589; this version posted January 19,2021 . The copyright holder for this preprint (which was not certified by peer review) is the author/funder, who has granted bioRxiv a license to display the preprint in perpetuity. It is made available under aCC-BY-NC-ND 4.0 International license.

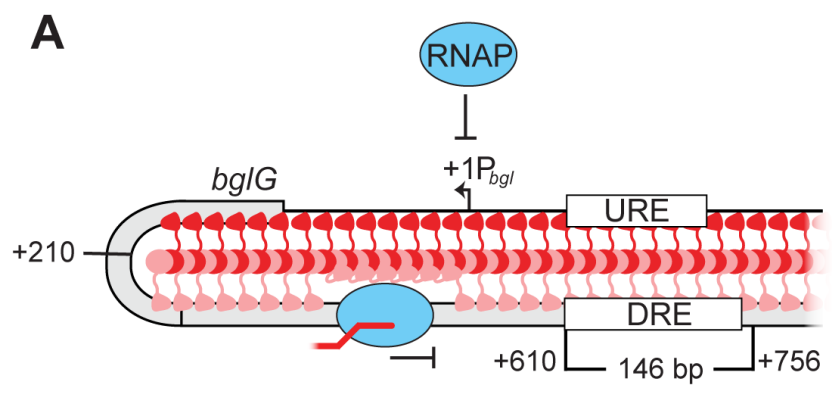

B

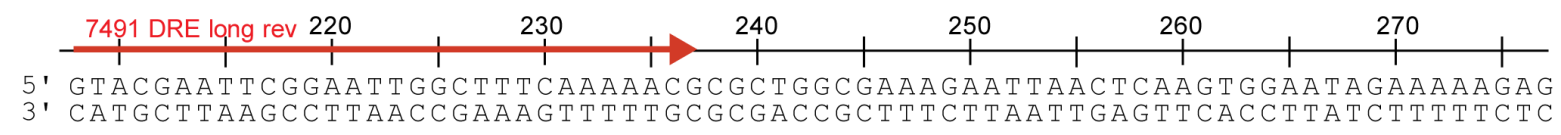

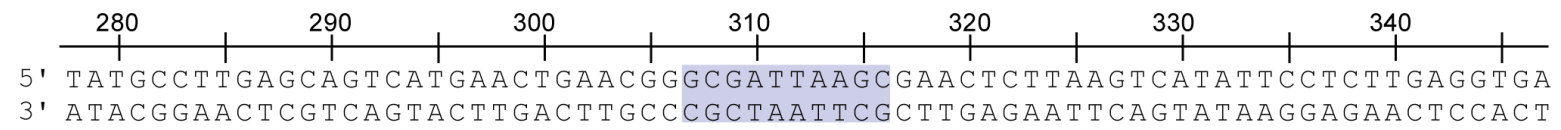

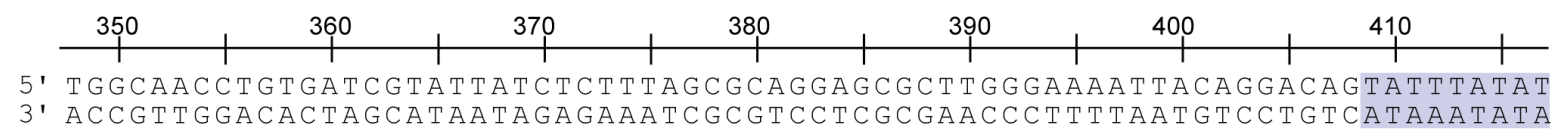

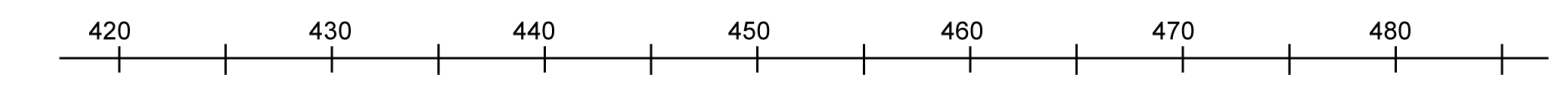

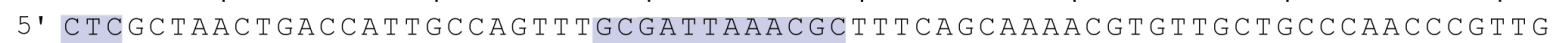
3' GAGCGATTGACTGGTAACGGTCAAACGCTAATT T GCGAAAGTCGTT T T GCACAACGACGGGT T GGGCAAC

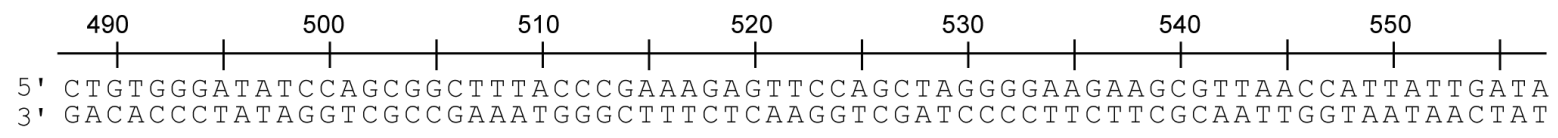

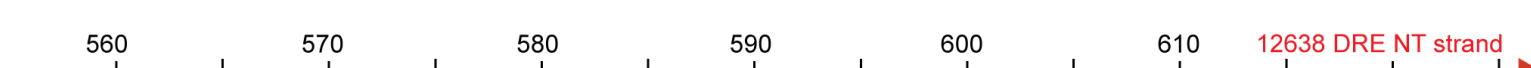
5' AACGGTTGGGGTGCAGT TACCGAAAGATGAATGGGCTTTATTGCCATGCATCTGGTCAGTGCCCAAAT 3. TTGCCAACCCGCACGTCAATG TTTCTACTTCACCCGAAATAACGGTACGTAGACCAGTCACGGGTTA

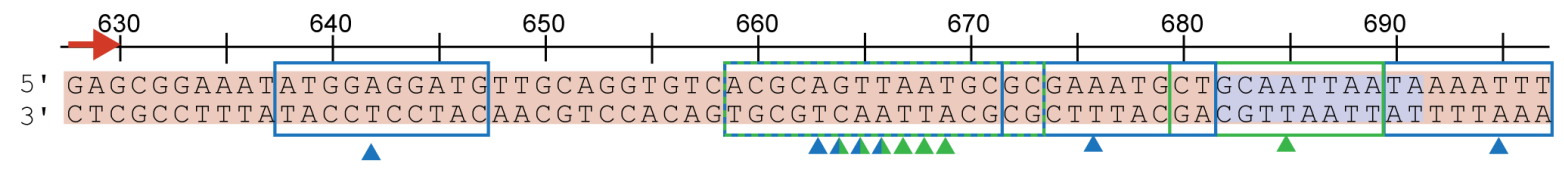

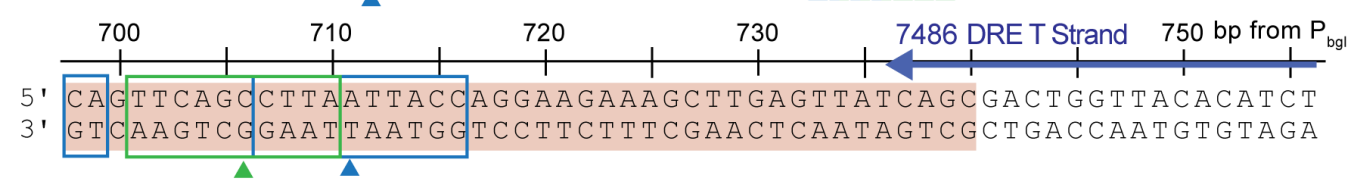

Predicted high-affinity H-NS binding site

146-bp DRE fragment

$\Delta$ Location of the center of the H-NS DBD

(matches black triangle in Figure 7B)
$\square \square$ TEN-map- \& $\cdot \mathrm{OH}$ footprint-determined H-NS DBD locations (facing either toward or away from $\mathrm{P}_{b g l}$ )

1304 Figure S4-1. DNA sequence of the $b g l$ operon region used for TEN-map experiments.

1305 (A) At the $b g l$ operon, an H-NS filament nucleates from both the downstream and upstream 1306 regulatory elements (DRE and URE; (Dole et al., 2004; Nagarajavel et al., 2007)), enabling 1307 formation of a bridged filament. A bridged filament occludes RNAP binding from the promoter 
and also can stimulate pausing by an elongating RNAP (Kotlajich et al., 2015; Rangarajan and Schnetz, 2018). Both mechanisms contribute to silencing of the $b g l$ operon. (B) The 146-bp DNA fragment was generated by PCR using $5^{\prime}{ }^{32} \mathrm{P}$-end-labeled primers to label either the nontemplate (NT) strand (red, primer \#12638) or the template (T) strand (blue, primer \#7486).

1312 Longer fragments (549 bp) were amplified with primers \#486 and \#7491 and labeled on the T

1313 strand. The location of the DRE as identified by (Dole et al., 2004) is highlighted in light pink. High-affinity H-NS binding sites identified by Virtual Footprint (http://www.prodoric.de/vfp/vfp_regulon.php) are indicated by blue filled boxes. The sequence is numbered by distance from the TSS of $\mathrm{P}_{b g l}$. Light blue and green open boxes highlight locations of H-NS DNA binding domains in Figure 7D. Triangles indicate where the center of the H-NS DBD (see black arrow in Figure $7 \boldsymbol{B}$ ) was placed at that sequence either toward (green) or away (blue) from the $b g l$ promoter (see Figure $8 \boldsymbol{A}$ ). 


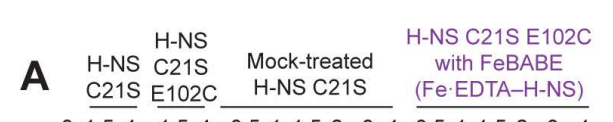
$0 \overline{1.54} \overline{1.54} \overline{0.511 .5234} \overline{0.511 .5234}$

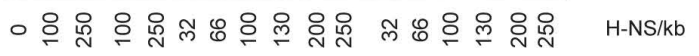

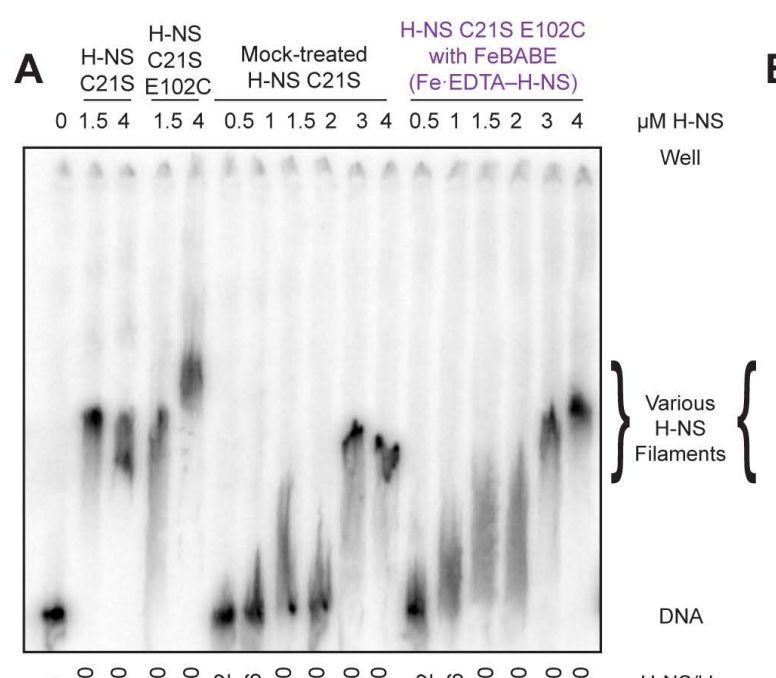

1321

1322

1323

1324

1325

1326

1327

1328

1329

1330

1331 rearranged for clarity.

Figure S4-2. Fe·EDTA-H-NS forms filaments on bgl DRE DNA.

Electrophoretic mobility shift assay showing filament formation on $10 \mathrm{nM} 1.5 \mathrm{~kb}$ DNA from the $b g l$ operon (Boudreau et al., 2018; Kotlajich et al., 2015) by H-NS C21S or H-NS C21S E102C with or without FeBABE treatment. H-NS C21S (unable to conjugate) was treated with FeBABE to account for loss of activity during conjugation process. Fe-EDTA-H-NS contains FeBABE conjugated to $\sim 50 \%$ of the E102C thiols in H-NS C21S E102C (see Methods). Panels A and B depict filament formation by two independent preparations of H-NS-FeBABE. Filaments formed were similar between preps, but the activity of H-NS varied between preparations. Panel (A) shows filament formation by sample one of conjugated H-NS and panel (B) shows filament formation by sample two of conjugated H-NS. All filaments were run on the same gel in (B), but 


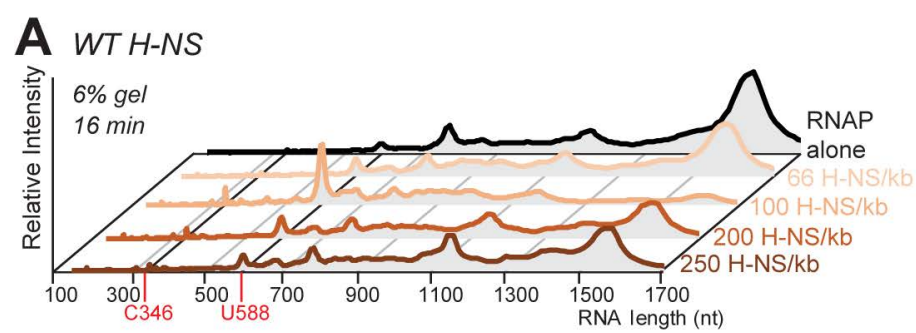

B C21SH-NS

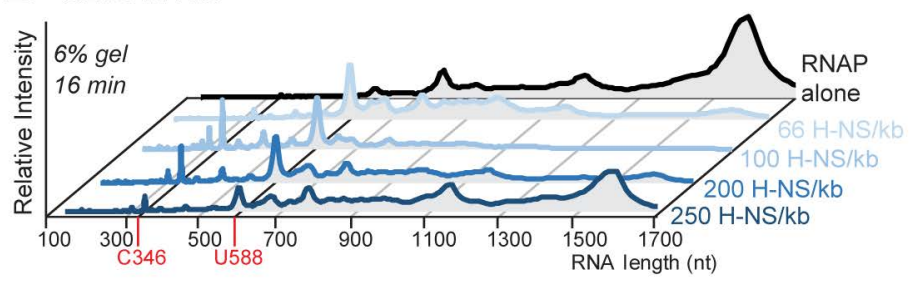

C C21SE102C H-NS

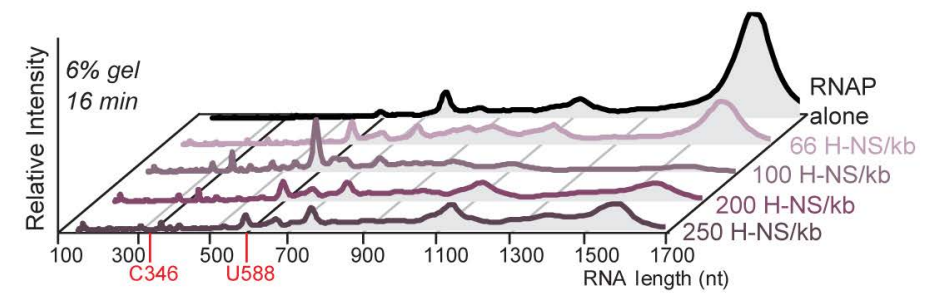

D Protein conjugated with FeBABE

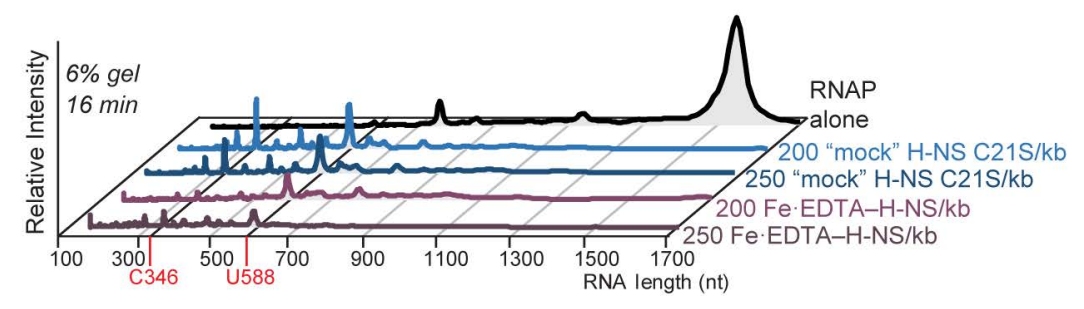

1333 Figure S4-3. Effect of H-NS variants and FeBABE conjugation to H-NS on elongating 1334 RNAP.

1335 Pseudo-densitometry traces show transcripts formed on a $1.5 \mathrm{~kb}$ bgl DNA by RNAP 16 min after transcription through various H-NS filaments is begun with $30 \mu \mathrm{M}$ NTPs: (A) WT H-NS filaments, (B) C21S H-NS filaments, (C) C21S E102C H-NS filaments, (D) either C21S (blue; "mock"-treated) or C21S E102C (purple; Fe-EDTA-H-NS) H-NS that were treated with FeBABE. Concentrations of protein that formed filaments were used for transcription assays (see

1340 Figure S4-2). 
A

A Strand

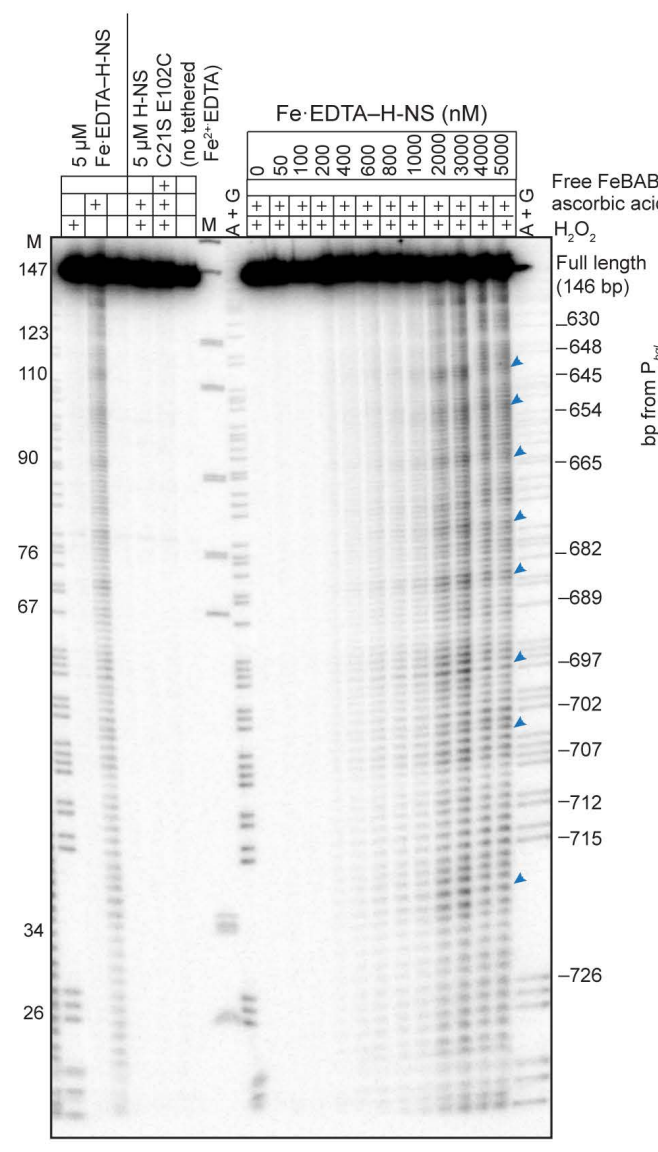

B Tethered T strand cleavage

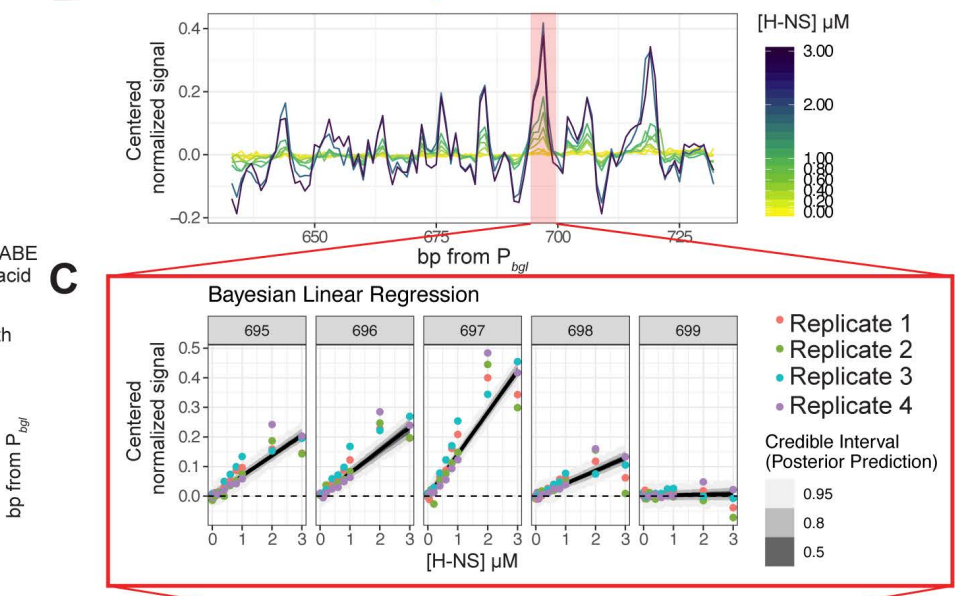

D H-NS dependence of T strand cleavage

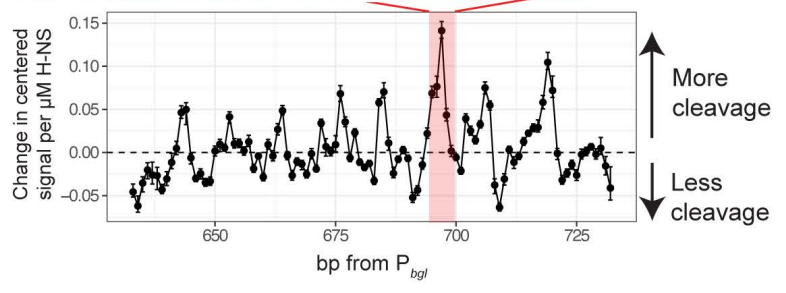

E Tethered NT strand cleavage
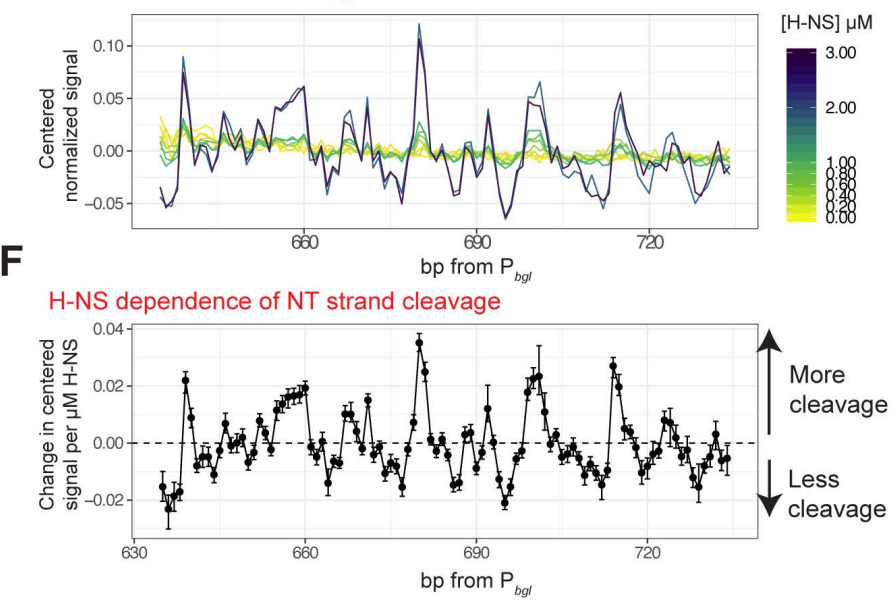

Figure S4-4. TEN-map analysis by linear regression of the Fe-EDTA-H-NS concentrationdependence of cleavage. (A) Denaturing 7\% PAG showing Fe-EDTA-H-NS cleavage pattern of 146-bp DRE DNA labeled on the T strand. Increasing concentrations of Fe.EDTA-H-NS were added to $\sim 20 \mathrm{nM}$ DNA. Cleavage was initiated with addition of $2 \mathrm{mM} \mathrm{H}_{2} \mathrm{O}_{2}$ and $2 \mathrm{mM}$ ascorbic acid. Controls we performed with only $2 \mathrm{mM} \mathrm{H}_{2} \mathrm{O}_{2}, 2 \mathrm{mM}$ ascorbic acid, water in the presence of $5 \mu \mathrm{M}$ Fe.EDTAH-NS (far left lanes). Addition of free FeBABE to filaments formed with $5 \mu \mathrm{M}$ H-NS C21S E102 that does not contain Fe-EDTA did not result in cleavage of the DNA (middle lanes). A+G indicates Maxam-Gilbert sequencing ladder. Blue arrows indicate hot spots of cleavage induced by Fe-EDTA-H-NS. SAFA peak area quantification from this gel are shown in Figure $\mathbf{4 F}$. (B) Cleavage of DRE DNA fragment $\mathrm{T}$ strand as a function of Fe-EDTA-H-NS concentration (yellow-to-purple gradient). Cleavage data were corrected for total signal in each lane and then normalized to the average peak area and centered on the mean. (C) The relationship between 
bioRxiv preprint doi: https://doi.org/10.1101/2020.06.11.146589; this version posted January 19,2021. The copyright holder for this preprint

(which was not certified by peer review) is the author/funder, who has granted bioRxiv a license to display the preprint in perpetuity. It is made available under aCC-BY-NC-ND 4.0 International license.

1355

1356

1357

1358

1359

1360

1361

mean-centered normalized peak areas and the Fe·EDTA-H-NS concentration (from 0 to $3 \mu \mathrm{M}$ ) on the T strand was determined for each base pair by using a Bayesian linear regression model. The example shows linear regression model fits for positions 695-699 on the T strand. (D) H-NS dependence of cleavage of the $\mathrm{T}$ strand as determined from Bayesian linear regression model. Error bars indicate credible intervals on the slope estimates. (E) Same as B, but for the NT strand. (F) Same as D, but for the NT strand. 
A

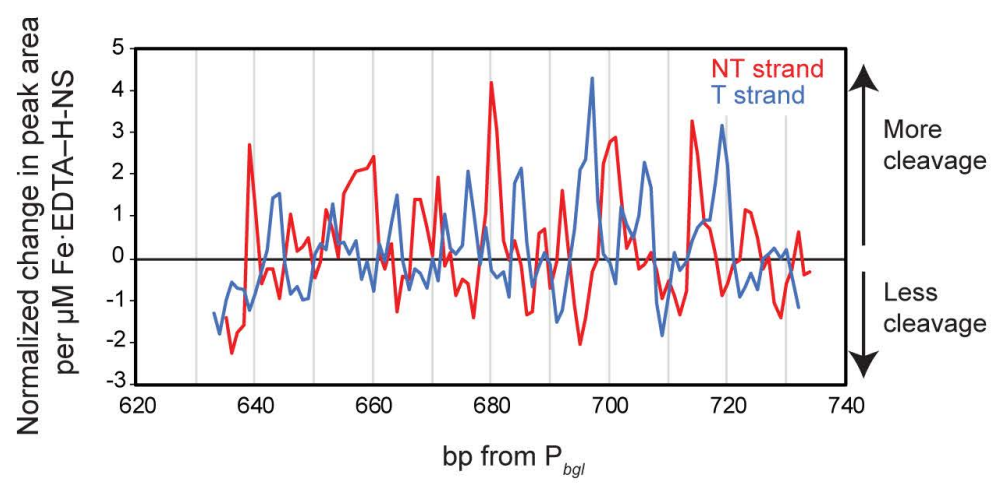

B

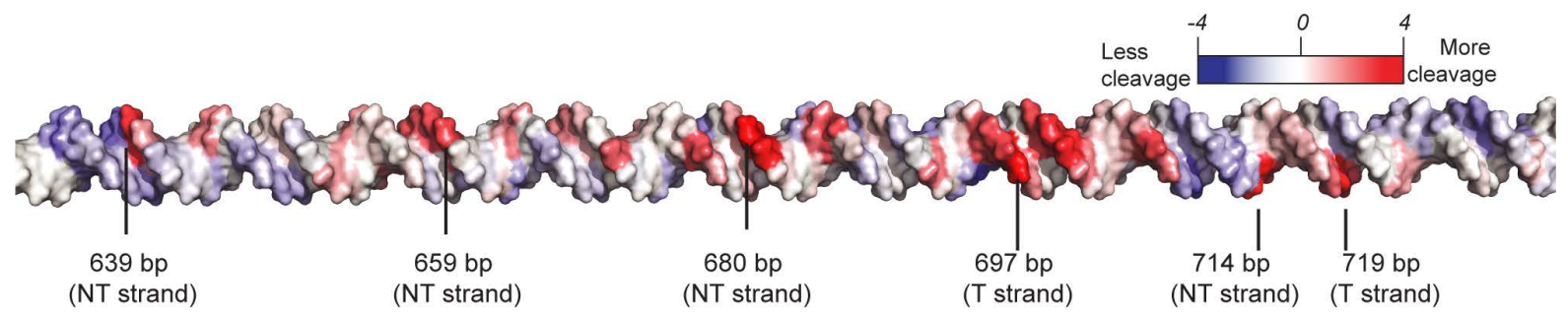

Figure S4-5. Use of linear regression-determined slopes to determine H-NS DBD locations. (A) Slope point-estimates (Figure $\boldsymbol{S 4}-\mathbf{4 C}$ and $\boldsymbol{F}$ ) were Robust-Z normalized within each strand for direct comparison between the strands (see Methods). More positive slopes indicate more HNS dependent cleavage at that $\mathrm{bp}$, indicating robust H-NS binding at the location. This Robust-Z normalized data was used to compare the TEN-map results and the Fe-EDTA-H-NS cleavage pattern predicted by MD simulation (Figure 7). (B) Data in (A) displayed on B-form DNA where red indicates the most-cut bases and blue are the least-cut bases. 


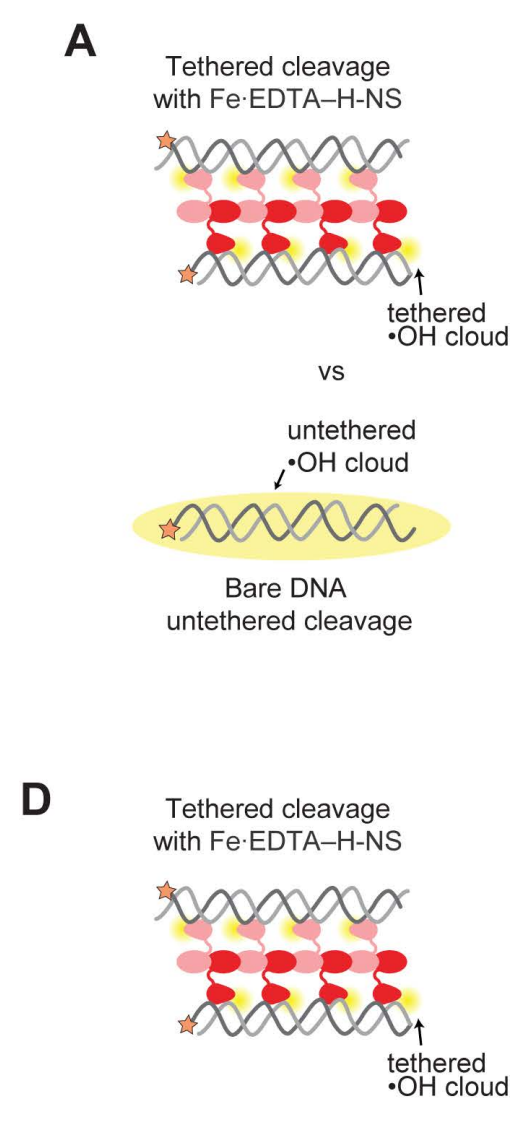

vs

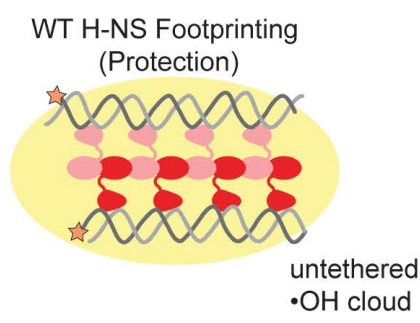

B

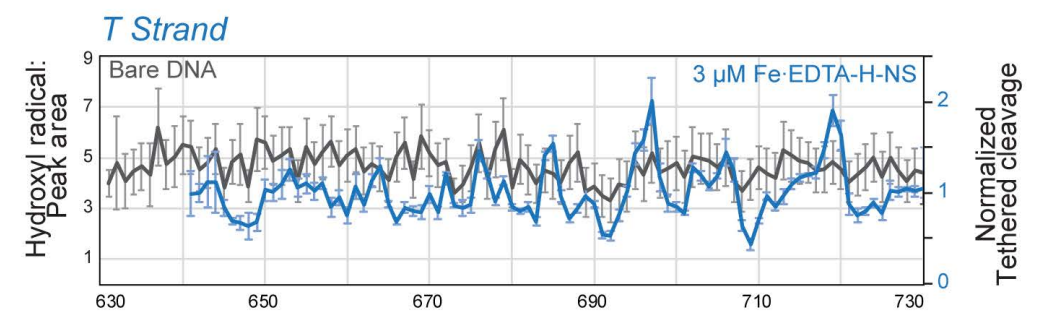

C
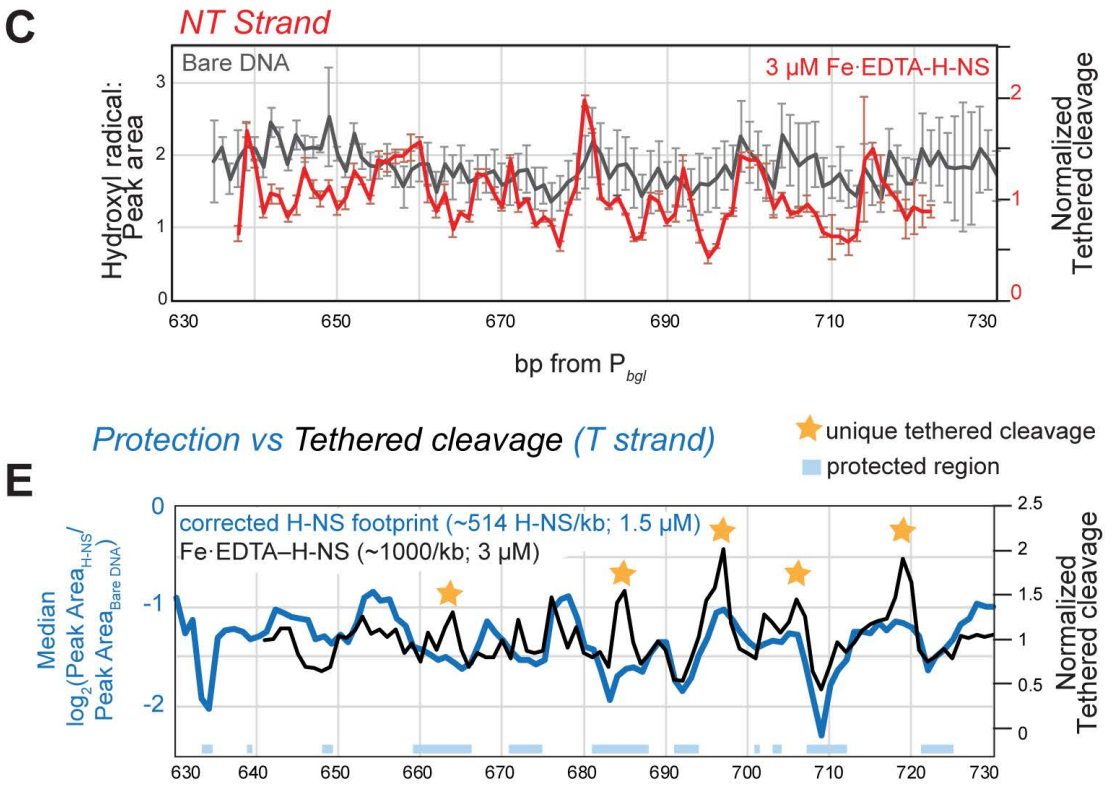

$\mathbf{F}$

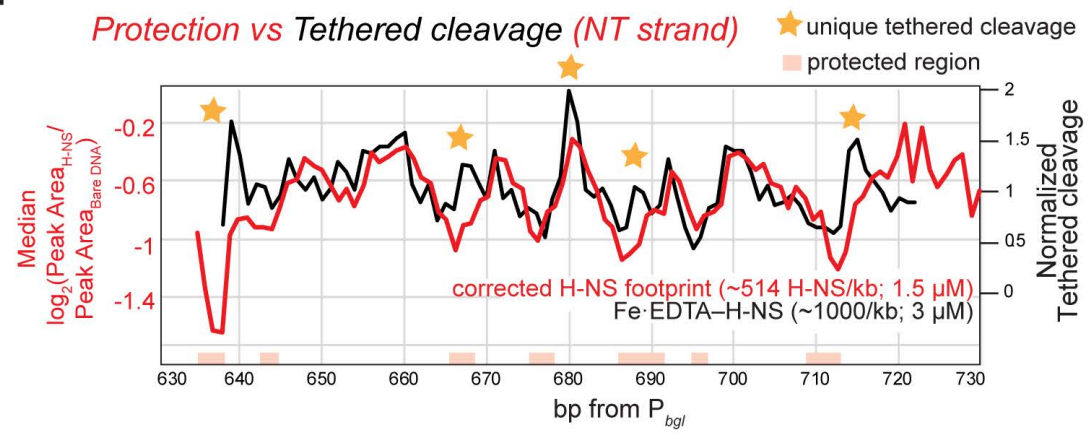

Figure S4-6. TEN-map cleavage patterns differ from freely diffusible $\cdot O H$ cleavage of bare DNA and of DNA in the presence of wild-type H-NS.

(A) To verify that TEN-map cleavage reveals H-NS binding sites, the tethered cleavage pattern possible reach of $\cdot \mathrm{OH}$ cleavage in the assay. Peak area of $\cdot \mathrm{OH}$ cleavage of 146-bp $\mathrm{bgl}$ DRE DNA T (B) or NT (C) strand in the absence of H-NS (gray) shows intrinsic cleavage propensity of the sequence used to form H-NS filaments. The intrinsic cleavage pattern of bare DNA (gray) differed from the tethered cleavage pattern generated by $3 \mu \mathrm{M}$ Fe-EDTA-H-NS on the T (blue) or NT (red) strand. The difference indicates specific cleavage by Fe-EDTA-H-NS. Error bars indicate standard deviation of three replicates. (D) Comparison of the TEN-map pattern (top) and the $\cdot \mathrm{OH}$ footprint (protection) pattern (bottom). Yellow ovals represent the possible reach of $\cdot \mathrm{OH}$ cleavage in the assay. (E) Blue line shows the $\cdot \mathrm{OH}$ footprints of wild-type $\mathrm{H}-\mathrm{NS}$ on the T strand corrected by subtracting the bare DNA cleavage pattern from the pattern of $\cdot \mathrm{OH}$ cleavage of the $\mathrm{T}$ strand in the presence of wild-type H-NS $(1.5 \mu \mathrm{M}$; Same trace as in Figure $6 C)$. Protected 
bioRxiv preprint doi: https://doi.org/10.1101/2020.06.11.146589; this version posted January 19,2021. The copyright holder for this preprint

(which was not certified by peer review) is the author/funder, who has granted bioRxiv a license to display the preprint in perpetuity. It is made available under aCC-BY-NC-ND 4.0 International license.

1385 regions (valleys in protection pattern) calculated from concentration-dependent slope estimates are indicated by shaded boxes (see Figure S6-2). Black line shows TEN-map cleavage by $\mathrm{Fe} \cdot \mathrm{EDTA}-\mathrm{H}-\mathrm{NS}(3 \mu \mathrm{M} ; 1027 \mathrm{H}-\mathrm{NS} / \mathrm{kb})$ demonstrating its distinct pattern, including cuts within

1389 H-NS footprints. The most notable cuts attributable to tethered Fe·EDTA are indicated by yellow 1390 stars. (F) Same as (E) but for the NT strand. 
A

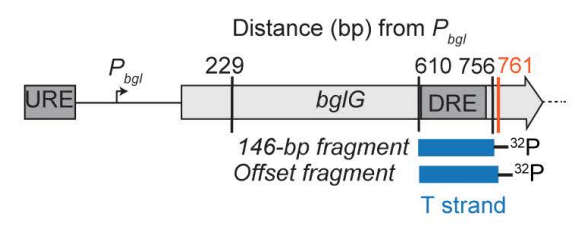

C

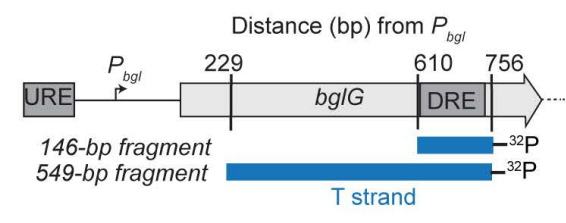

\section{B}
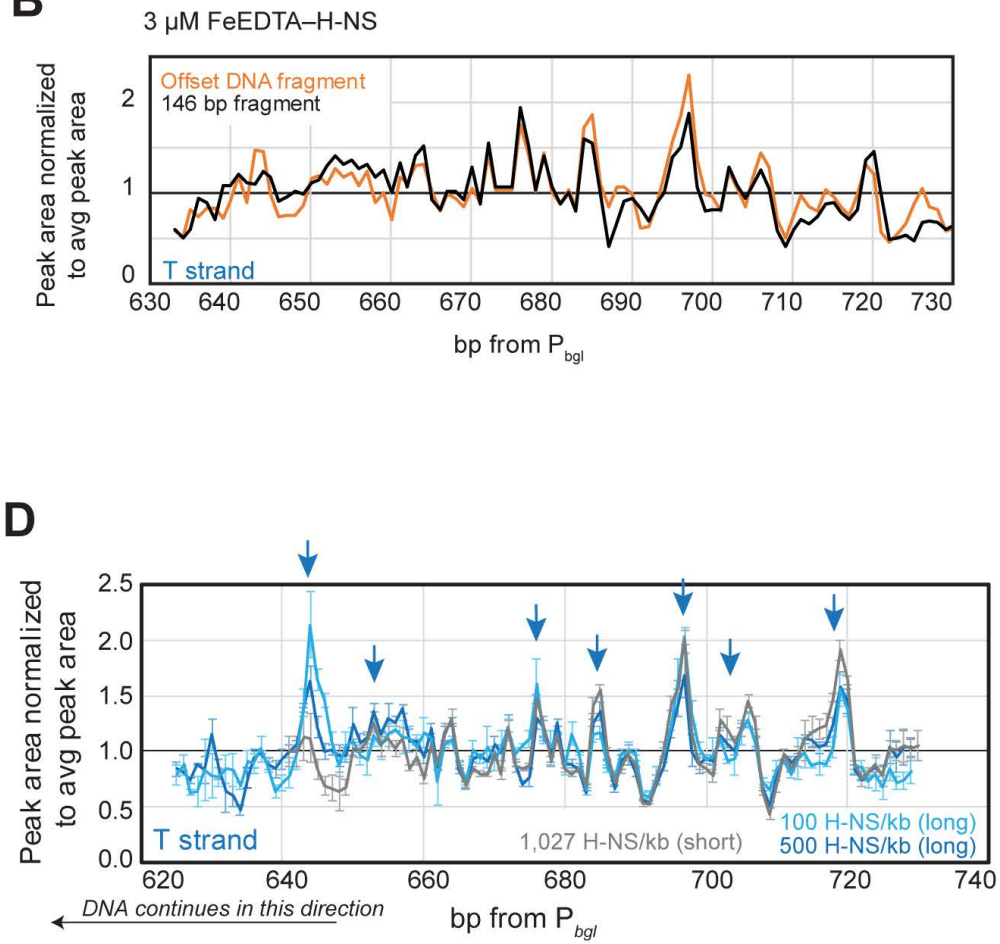

Figure S4-7. TEN-map cleavage is unaffected by flanking DNA context.

(A) Schematic of the DNA templates used to test if H-NS binding is sequence-specific or initiates from the end of the DNA. The 146-bp bgl DRE fragment (black arrow) was used in experiments shown in Figure 4. An alternative PCR primer (orange line) was used to shift the DNA end by 5 bp relative to the standard 146-bp fragment. (B) TEN-map cleavage peak areas for the 146-bp fragment (black) and the 151-bp offset DNA fragment (orange). Cleavage and quantification were performed as described in Methods (averages from two experiments). (C) Comparison of the standard (146 bp) and long (549 bp) DNA fragments generated from the bgl operon to test effects of DNA length on arrangement of H-NS DBD within a filament. Both fragments were labeled on the T strand. (D) TEN-map cleavage patterns for standard (gray) or long (blue) DNAs. Gray line matches data in Figure $\mathbf{4 F}$. To form filaments on long DNA, H-NS a mixture of $60 \%$ wild-type H-NS and $40 \% \mathrm{Fe}$-EDTA-H-NS was used because the concentration of Fe·EDTA-H-NS alone was too low to form pure Fe-EDTA-H-NS filaments. Error bars show standard deviation of three replicates. Arrows indicate peaks of cleavage. 
A Effect of constrained bridging

\section{B Effect of Hha}

Fe-EDTA-H-NS filaments
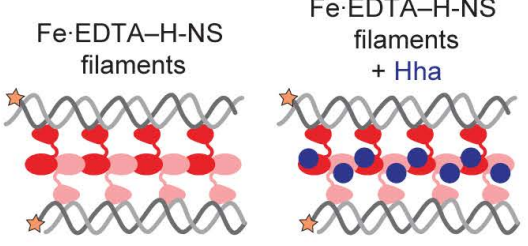
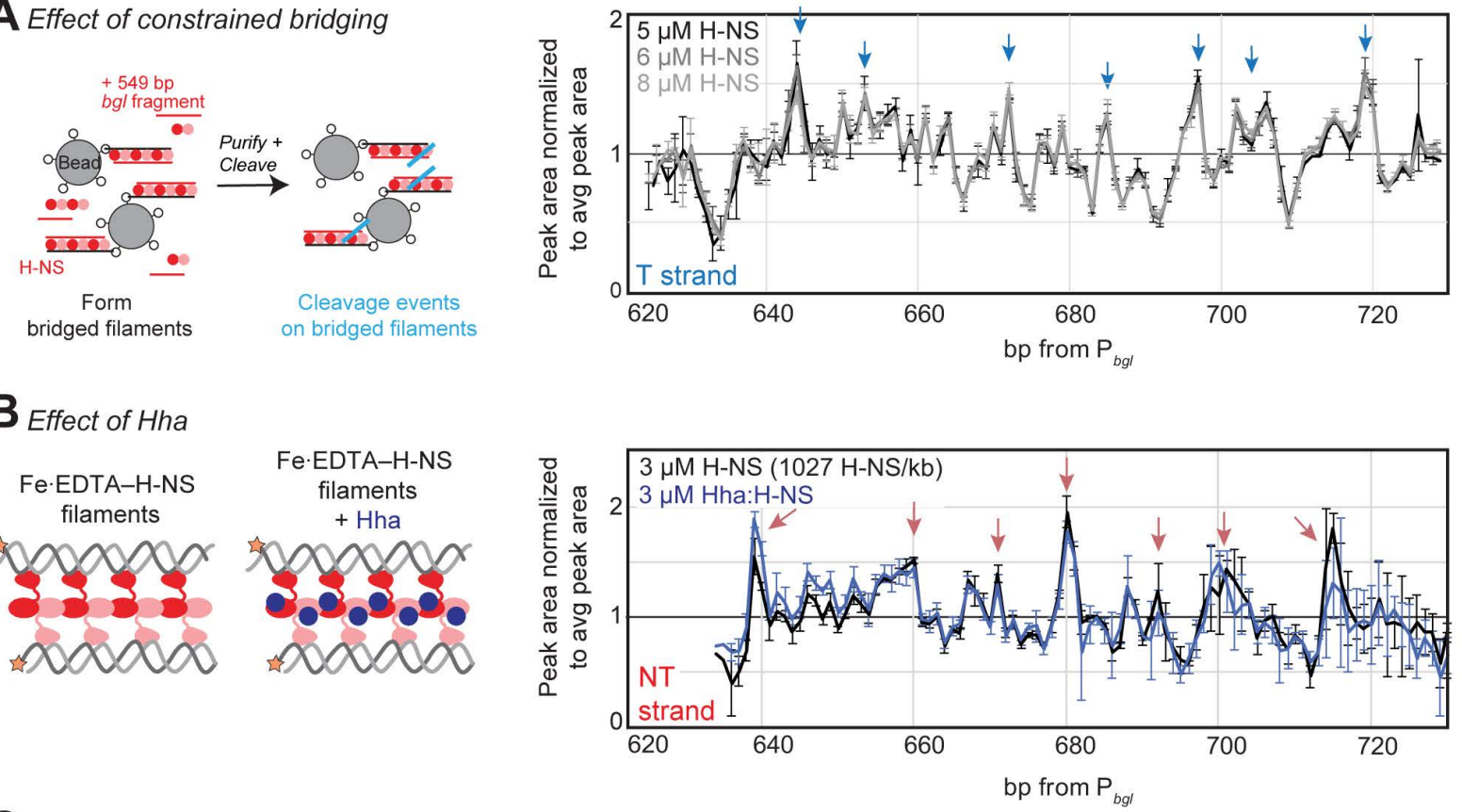

C Effect of $\mathrm{Mg}^{2+}$

Fe-EDTA-H-NS

+ WT filaments
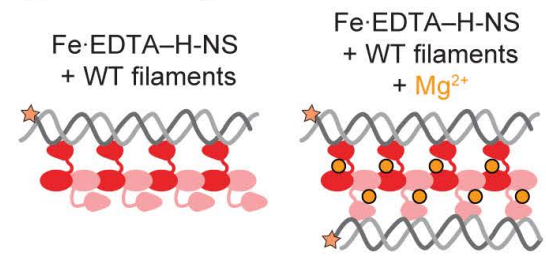

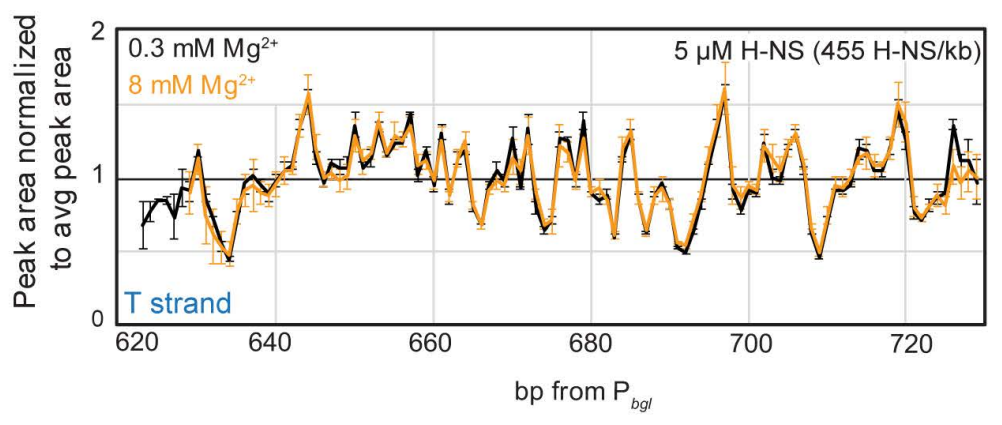

Figure 5. TEN-map cleavage of $b g l \mathrm{DRE}$ is unaffected by obligate bridging, $\mathrm{Hha}$, or $\mathrm{Mg}^{2+}$ concentration.

(A) TEN-map cleavage of $b g l$ DRE is unaffected by obligate bridging. Bridged filaments were formed by mixing unlabeled 685-bp DNA fragment bound to paramagnetic beads (see Methods), 549-bp bgl DNA labeled on the T strand, and H-NS ( $60 \%$ wild-type H-NS $+40 \% \mathrm{Fe} \cdot \mathrm{EDTA}-\mathrm{H}-$ NS) at the indicated concentrations. Bridged filaments were recovered by magnetic separation and resuspended in cleavage buffer to initiate cleavage of DNA (Figure S5-2; see Methods). TEN-map cleavage of a portion of the obligately bridged T strand is shown. (B) TEN-map cleavage of $b g l$ DRE is unaffected by Hha. Formation of pure H-NS filament or an H-NS filament mixed with equimolar Hha (blue circle) was confirmed by EMSA and in vitro transcription assay (Figure S5-3). TEN-map cleavage of the $b g l$ DRE NT in the presence (blue) or absence (black) of equimolar Hha. Red arrows indicate hotspots of cleavage, which are at the same locations for both H-NS and Hha:H-NS filaments. (C) TEN-map cleavage of $b g l$ DRE is unaffected by $\mathrm{Mg}^{2+}$ concentration. TEN-map cleavage of T strand at $5 \mu \mathrm{M}(455 \mathrm{H}-\mathrm{NS} / \mathrm{kb})$ in the presence $8 \mathrm{mM} \mathrm{Mg}^{2+}$ (orange) or $0.3 \mathrm{mM} \mathrm{Mg}^{2+}$ (black). Filaments were formed on $549 \mathrm{bp}$ fragment from the $b g l$ operon. H-NS was added at a 60-40 mixture of wild-type and Fe EDTAH-NS to match experiments done on beads in panel A. In all data panels, hotspots of TEN-map cleavage are indicated by arrows and error bars represent average of at least three replicates. Associated data: Figure S5-1, Figure S5-2, Figure S5-3, and Figure S5-4. 


\section{A}
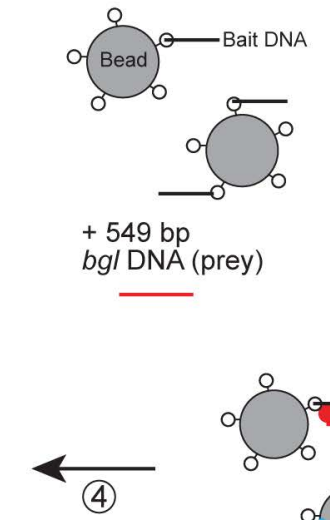

Stop cleavage \& visualize
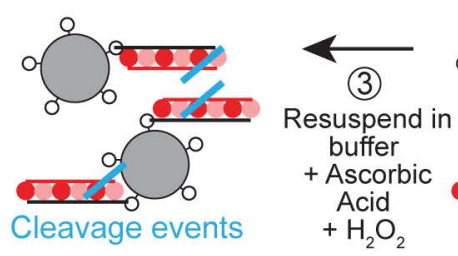

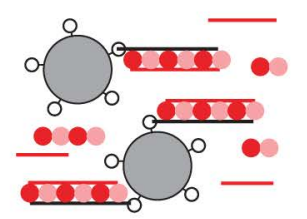

(2)

Purify bridged filaments

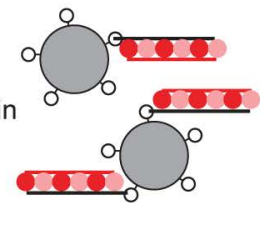

B

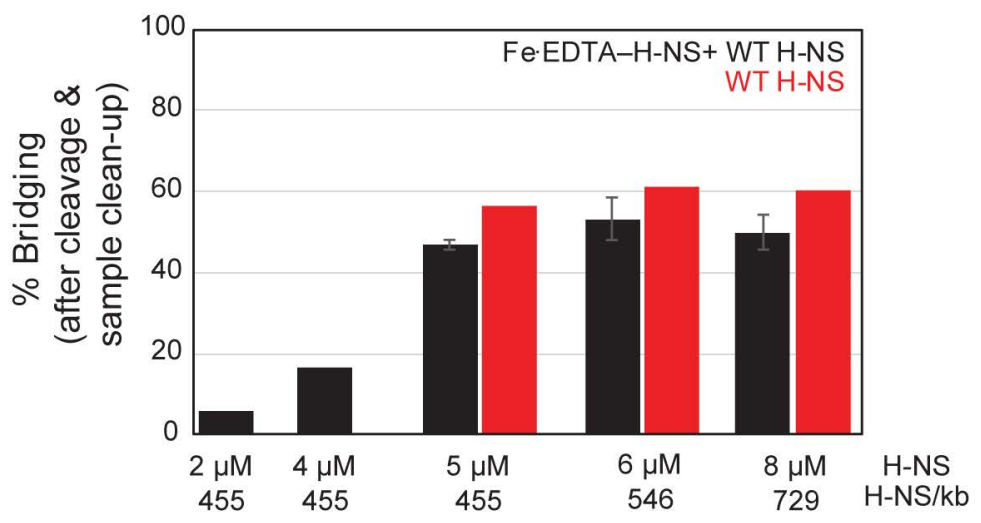

Figure S5-1. TEN-map cleavage of obligately bridged filaments formed on beads.

(A) Set-up of bridged cleavage assay. Filaments were formed with a $60-40$ mix of wild-type HNS and Fe-EDTA-H-NS over a range of concentrations (5, 6 and $8 \mu \mathrm{M} \mathrm{H}-\mathrm{NS}$ or 455, 546, and $729 \mathrm{H}-\mathrm{NS} / \mathrm{kb}$ ) in the presence of streptavidin paramagnetic beads containing immobilized 685bp DNA (van der Valk et al., 2017). H-NS monomers (conjugated or wild-type) are shown as alternating pink and red spheres to indicate head-head, tail-tail dimerization. The 549-bp bgl DRE DNA (prey DNA) was not tethered to beads and was labeled on the T strand. Bridged filaments were recovered by magnetic separation and gently resuspended in $20 \mu \mathrm{L}$ Filament Buffer (see Methods) followed by rapid addition of $1 \mu 150 \mathrm{mM}$ ascorbic acid and $4 \mu \mathrm{L} 12.5 \mathrm{mM}$ $\mathrm{H}_{2} \mathrm{O}_{2}$ to induce cleavage. Reactions were stopped with $22.4 \mathrm{mM}$ thiourea, extracted with phenol, recovered by ethanol precipitation, and separated by denaturing 7\% PAGE. (B) The percentage of prey DNA recovered by bridging in the presence of either wild-type H-NS (red) or a mixture of wild-type H-NS and Fe·EDTA-H-NS (black). 
bioRxiv preprint doi: https://doi.org/10.1101/2020.06.11.146589; this version posted January 19,2021 . The copyright holder for this preprint (which was not certified by peer review) is the author/funder, who has granted bioRxiv a license to display the preprint in perpetuity. It is made available under aCC-BY-NC-ND 4.0 International license.

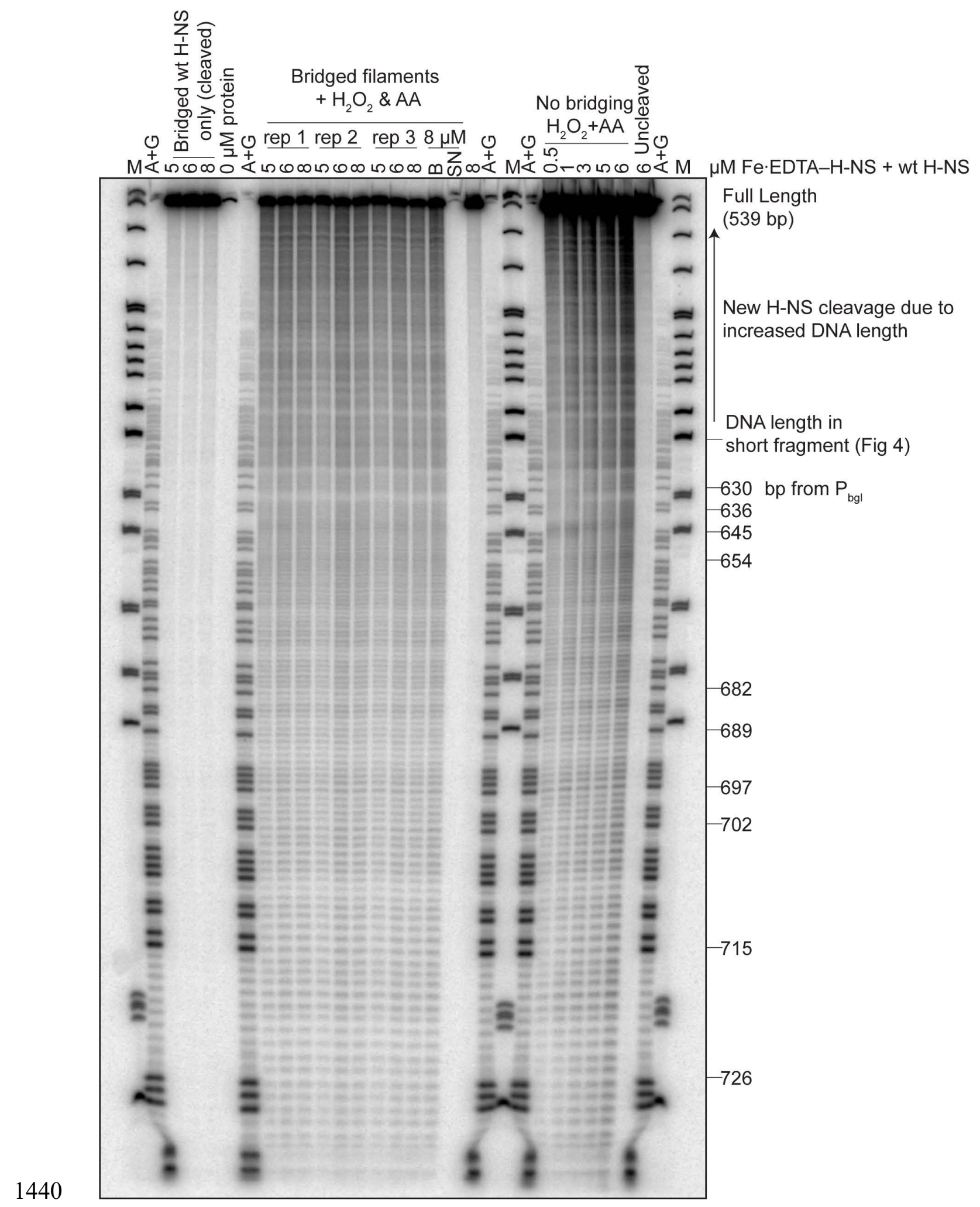

1441 Figure S5-2. TEN-map cleavage of obligately bridged DNA versus in solution.

1442 Denaturing 7\% PAG showing cleavage products generated by Fe-EDTA-H-NS in filaments 1443 formed on beads (obligately bridged filaments) or in solution (no obligate bridging). H-NS used 1444 to form filaments was $40 \%$ Fe-EDTA-H-NS and $60 \%$ wild-type H-NS at the indicated 
1445 concentrations. $8 \mu \mathrm{M}$ H-NS lanes marked "B" and "SN" indicate filaments formed with $8 \mu \mathrm{M} \mathrm{H}$ 1446 NS, cleaved, and then magnetically separated into supernatant (SN), containing DNA that dissociated from the beads, and bound fractions ("B", obligately bridged). Non-obligately bridging filaments were formed on the 545-bp $b g l$ DNA without beads or bait DNA present at indicated concentrations of $\mathrm{H}-\mathrm{NS}$. A+G, Maxam-Gilbert A and G sequencing ladder. M, MspI digest pBR322. 

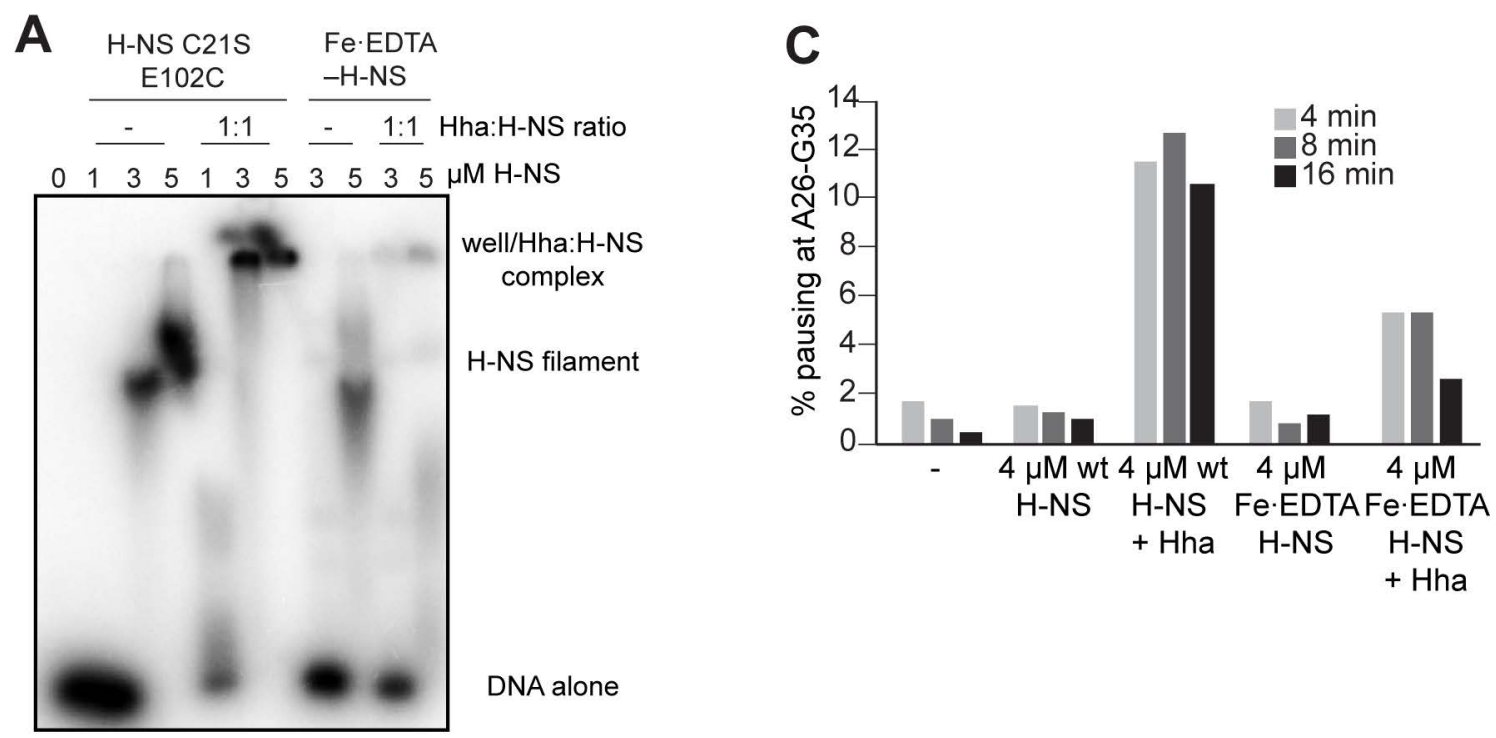

B

8 min after addition of NTPS

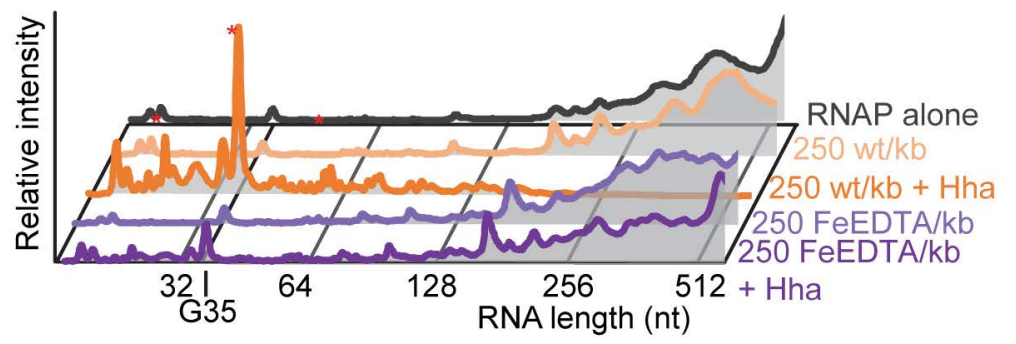

Figure S5-3. Properties of Hha:H-NS filaments formed with Fe-EDTA-H-NS. (A) Native 3\% PAG showing H-NS or Hha:H-NS filament formation on 146-bp DRE DNA. Amounts of H-NS C21S E102C or Fe·EDTA-H-NS were as indicated on the figure. Hha:H-NS filaments are retained in the well as previously observed (Boudreau et al., 2018). (B) Pseudodensitometry plot showing transcripts produced by RNAP 8 min after addition of $30 \mu \mathrm{M}$ NTPs in the presence of H-NS or Hha:H-NS filaments formed with wild-type or Fe·EDTA-H-NS. Pauses stimulated by Hha:H-NS are indicated with red asterisks. (C) Amount of pausing stimulated by H-NS and Hha:H-NS filaments. Hha added to both wild-type and Fe-EDTA-H-NS stimulated pausing at positions between A26 and G35 (Boudreau et al., 2018). 


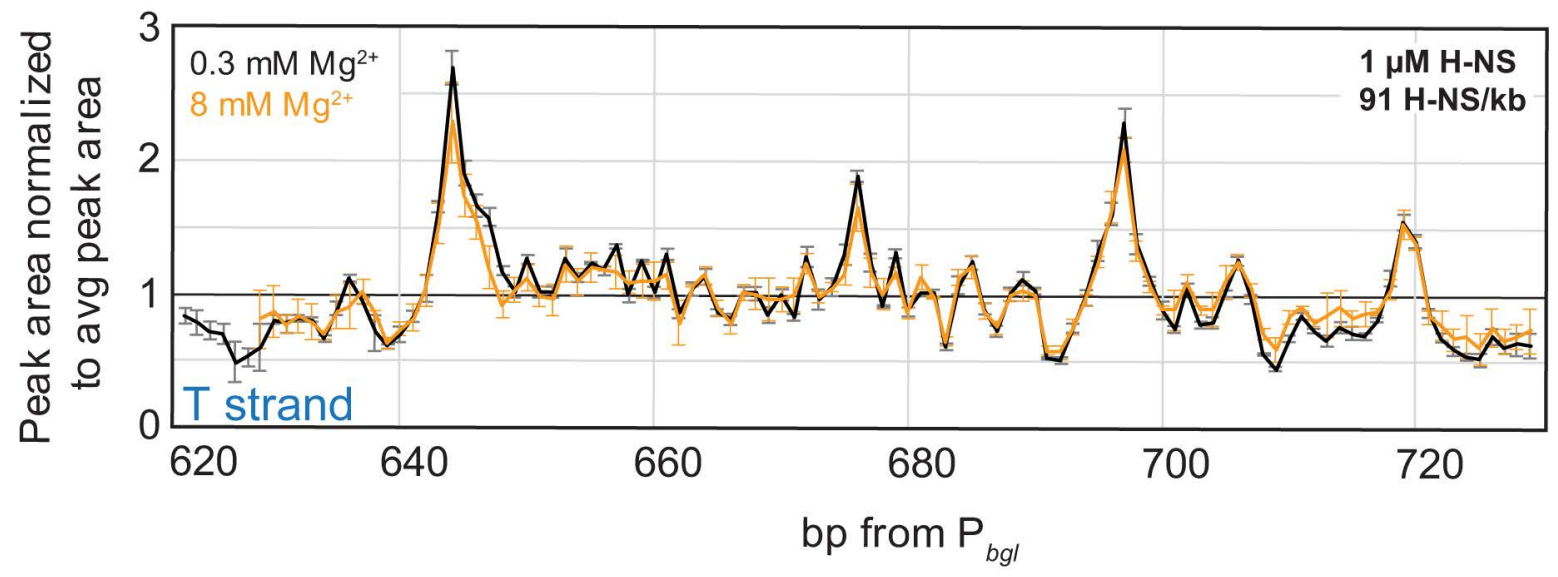

1462 Figure S5-4. H-NS DBDs bind DNA similarly at low and high $\mathbf{M g}^{2+}$.

1463 TEN-map cleavage pattern of the $b g l$ DRE 146-bp fragment ( ${ }^{32} \mathrm{P}$-labeled T strand) at $1 \mu \mathrm{M} \mathrm{H}-\mathrm{NS}$ 1464 1465 1466 1467 $\left(91 \mathrm{H}-\mathrm{NS} / \mathrm{kb}\right.$ ) in the presence $8 \mathrm{mM} \mathrm{Mg}^{2+}$ (black) or $0.3 \mathrm{mM} \mathrm{Mg}^{2+}$ (orange). Filaments were formed on $549 \mathrm{bp}$ fragment from the $b g l$ operon labeled on the T strand. H-NS added was a 60 40 mixture of wild-type and Fe-EDTA-H-NS to match experiments done on beads (Figure 5A). 

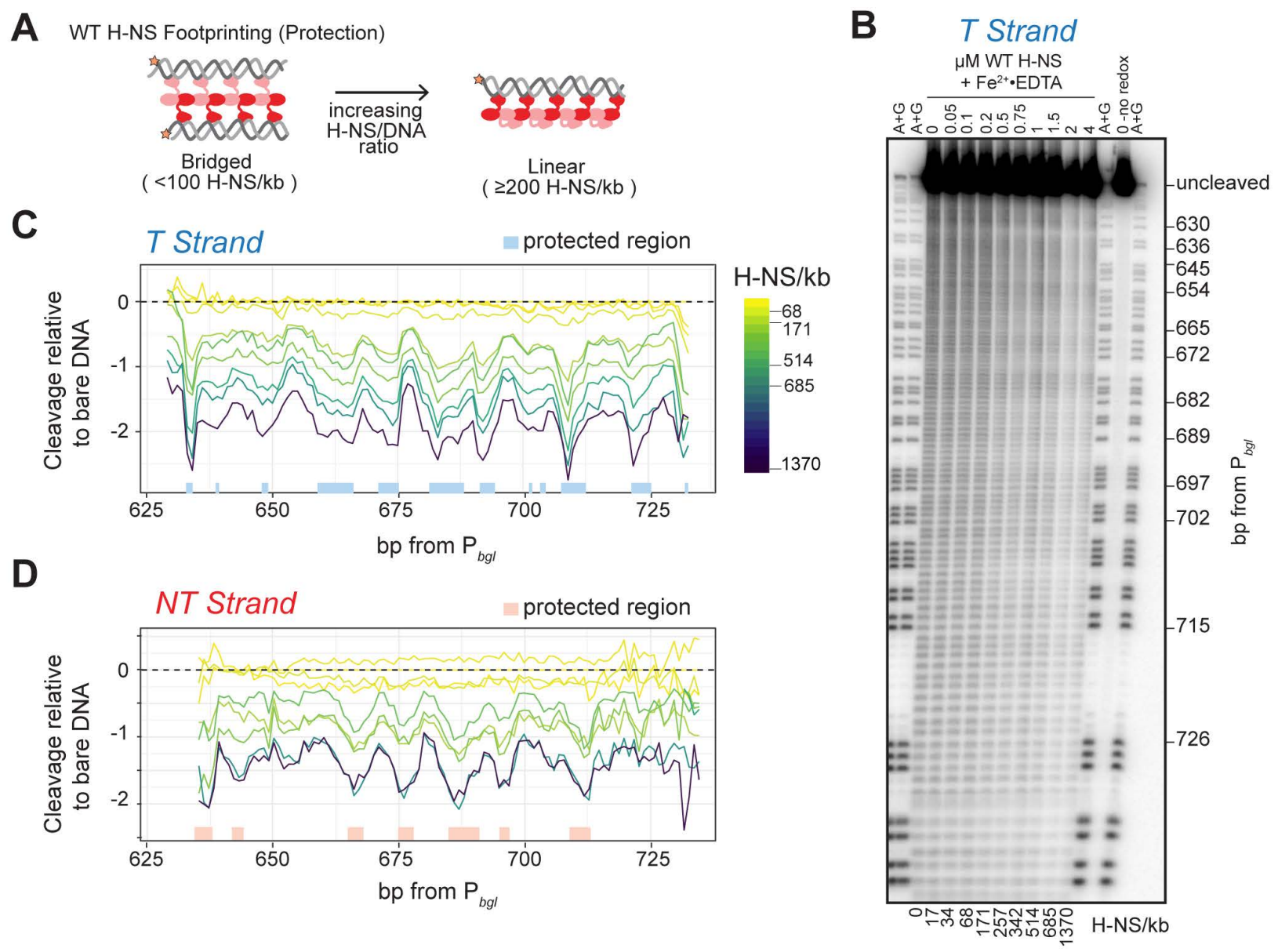

Figure 6. H-NS DBDs do not change locations during the switch of bridged-to-linear filaments as reported by $\cdot \mathrm{OH}$ footprinting.

(A) Schematic showing traditional $\cdot \mathrm{OH}$ footprinting with either bridged or linear $\mathrm{H}-\mathrm{NS}$ filaments. In traditional footprinting, excess $\mathrm{Fe}^{2+}$. EDTA is added in solution to create a pool of free radicals that diffuse freely so that any DNA not bound by wild-type H-NS will be cleaved (Figure S4-6). (B) Denaturing 7\% PAG showing hydroxyl radical cleavage pattern of 146-bp bgl DRE DNA ( ${ }^{32} \mathrm{P}$-labeled on the T strand) in the presence or absence of wild-type H-NS filaments formed at $0.05,0.1,0.2,0.5,0.75,1,1.5,2$, or $4 \mu \mathrm{M} \mathrm{H}-\mathrm{NS}(17,34,68,171,251,342$, $514,685,1370 \mathrm{H}-\mathrm{NS} / \mathrm{kb}$ ). (C) The footprinting pattern on the T strand was quantified by normalizing the cleavage of DNA in the presence of H-NS to cleavage of bare DNA, and then $\log _{2}$ transformed. Protected regions (shaded boxes) were assigned based on the positions exhibiting the greatest change in protection as a function of H-NS concentration (Figure S6-1). (D) Same as C, but for the NT strand. Lines represent the median of 4 experiments, but error bars are removed for clarity. Associated data: Figure S6-1 and Figure S6-2. 
bioRxiv preprint doi: https://doi.org/10.1101/2020.06.11.146589; this version posted January 19,2021 . The copyright holder for this preprint (which was not certified by peer review) is the author/funder, who has granted bioRxiv a license to display the preprint in perpetuity. It is made available under aCC-BY-NC-ND 4.0 International license.

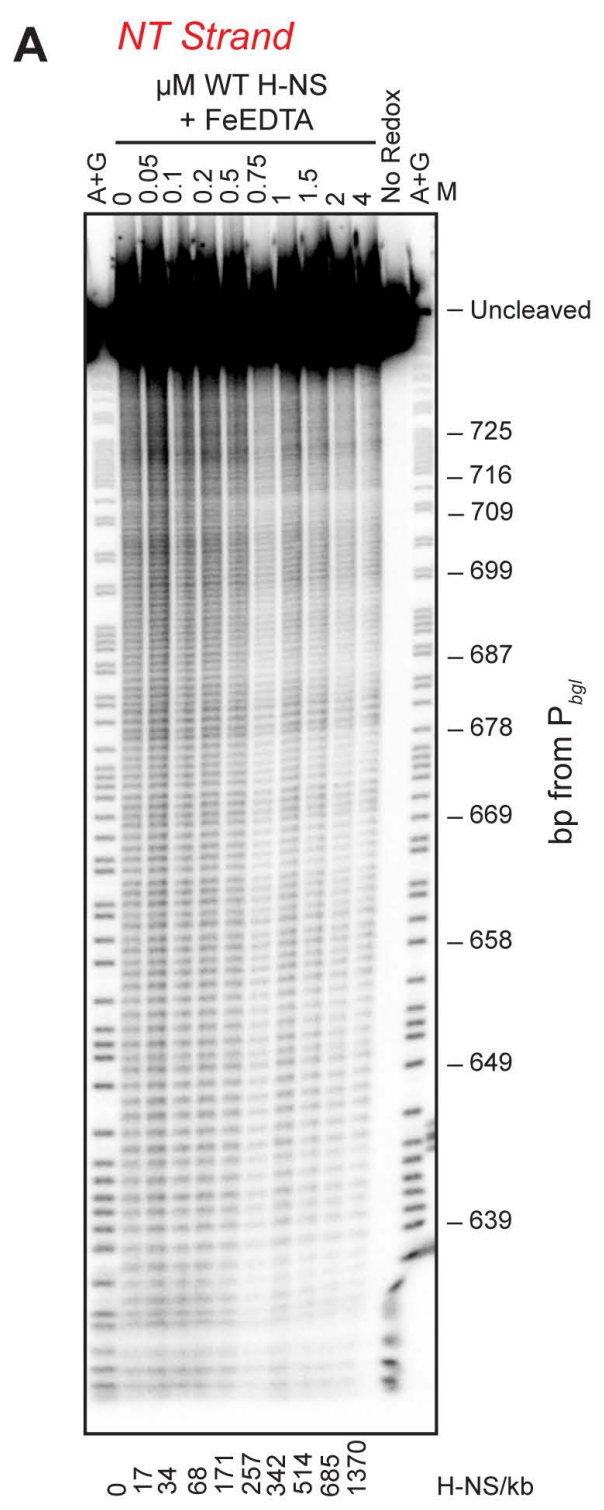

1485

1486 1487 1488 1489 1490 1491 1492 1493 1494 1495

\section{models.}

B

Protection of T strand (cleavage normalized to bare DNA)

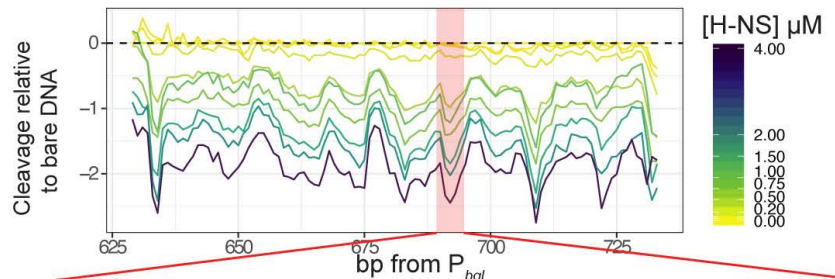

C

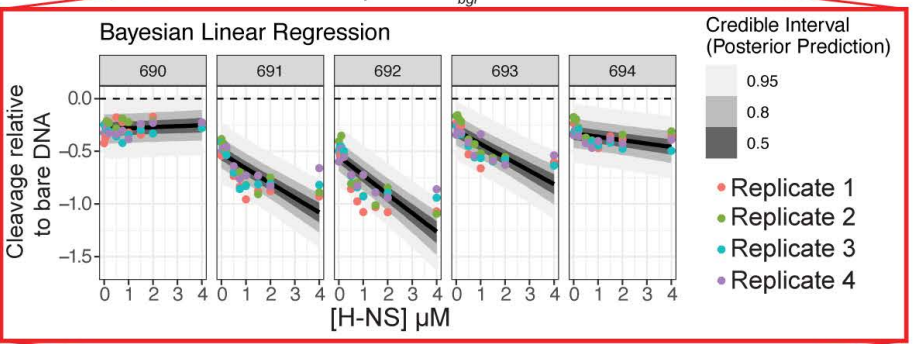

D H-NS dependence of $T$ strand protection

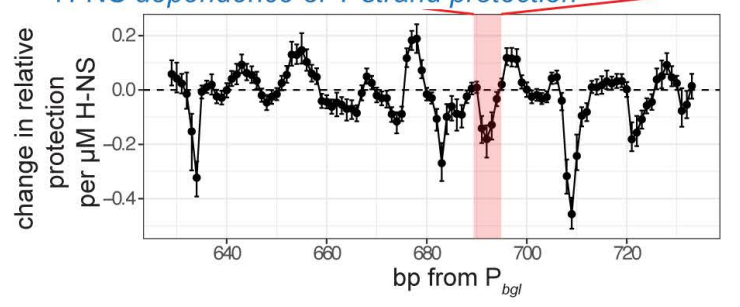

E

Protection of NT strand (cleavage normalized to bare DNA)

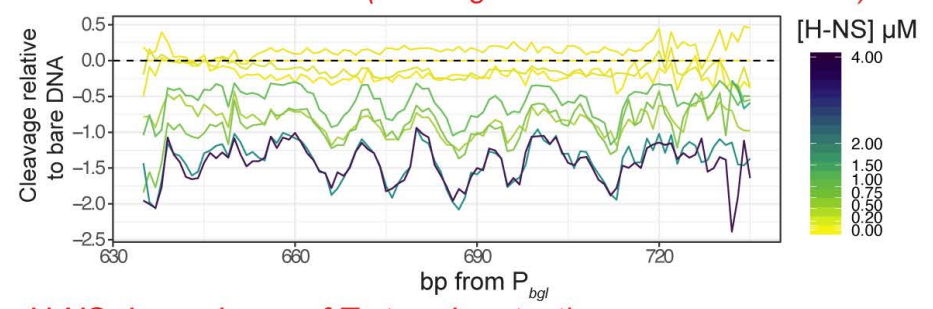

F H-NS dependence of T strand protection

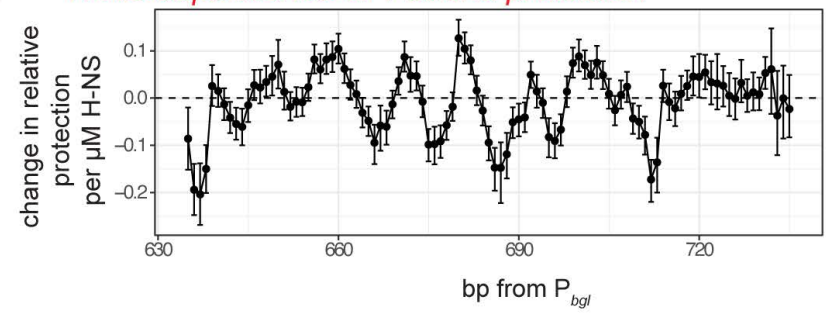

\section{Figure S6-1. Analysis of $\cdot \mathrm{OH}$ footprinting of $\mathrm{H}-\mathrm{NS}$ filaments using linear regression}

(A) Denaturing 7\% PAG showing hydroxyl radical cleavage pattern of 146-bp $b g l$ DRE DNA ( ${ }^{32} \mathrm{P}$-labeled on the NT strand) in the presence or absence of wild-type H-NS filaments formed at $0.05,0.1,0.2,0.5,0.75,1,1.5,2$, or $4 \mu \mathrm{M} \mathrm{H}-\mathrm{NS}$. The ratio of $\mathrm{H}-\mathrm{NS} / \mathrm{kb}$ is indicated below the gel. Wild-type H-NS plus untethered Fe-EDTA lanes were generated by sequential addition of $\mathrm{H}_{2} \mathrm{O}_{2}$, ascorbic acid, and Fe·EDTA. Gels are representative of at least 3 experiments. (B) Same as the data shown in Figure $\mathbf{6} \boldsymbol{C}$. The $\cdot \mathrm{OH}$ footprinting pattern for $\mathrm{H}-\mathrm{NS}$ was quantified by calculating the change in cleavage of each position compared to cleavage of bare DNA and then $\log _{2}$ transformed. The median of 4 replicates was calculated, but error bars are removed for 
1496 clarity. (C) For all positions, normalized peaks areas shown in (B) were plotted as a function of 1497 H-NS concentration. The change in peak area as a function of H-NS concentration (slope) was calculated using a Bayesian linear regression model. Example linear regression model fits shown for positions 690-694 on the T strand. (D) Change in protection relative to bare DNA as a function of H-NS concentration for the T strand. Error bars indicate $95 \%$ credible intervals on the slope estimates. (E) Same as B, but for the NT strand. Data also shown in Figure 6D. (F) 
B
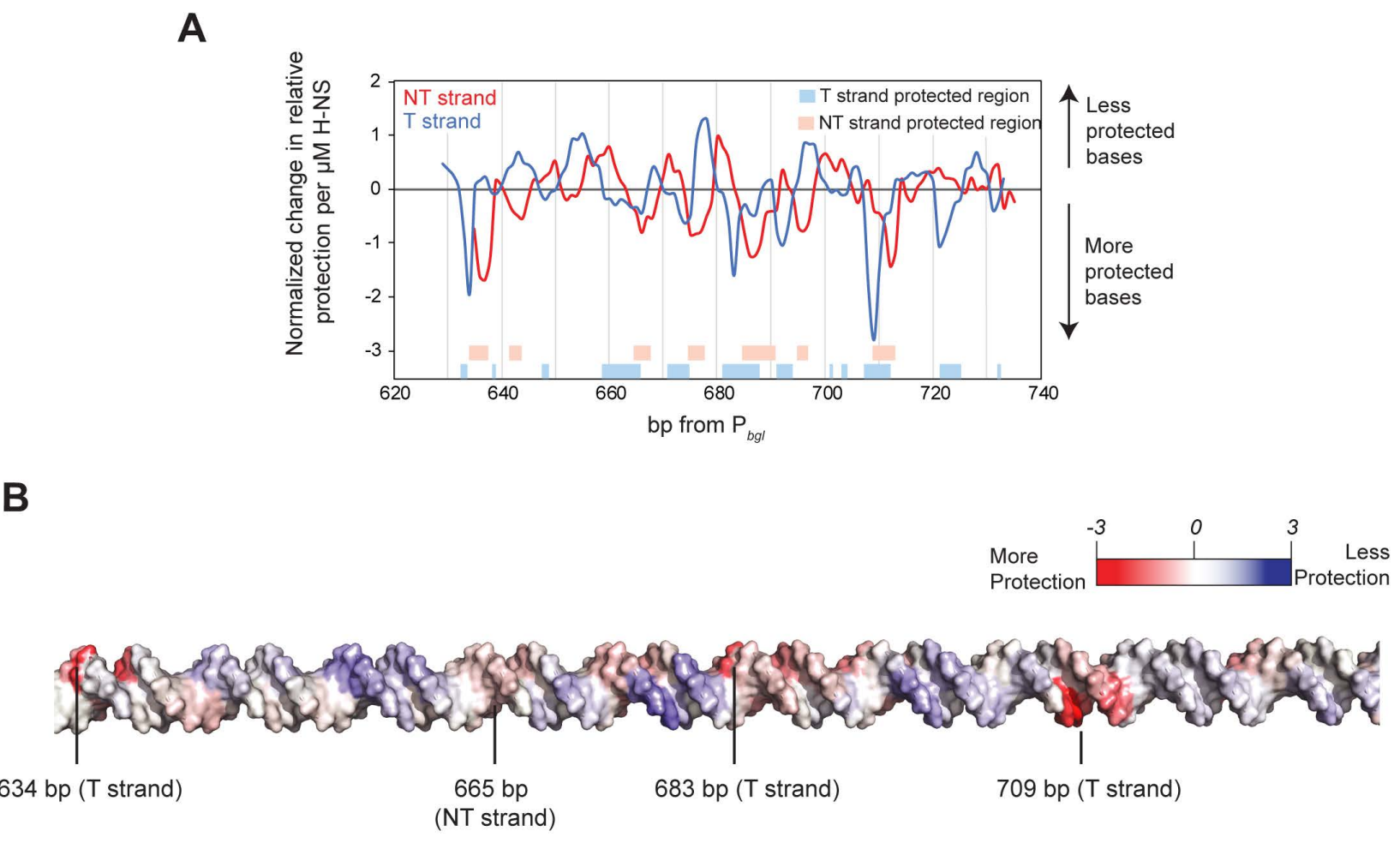

\section{Figure S6-2. Sites of protection from · OH cleavage by wild-type H-NS reveal DBD-DNA contacts in both bridged and linear filaments.}
(A) Slope point-estimates (Figure S6-1D and $\boldsymbol{F}$ ) were Robust-Z normalized within each strand for direct comparison between the strands (see Methods). More negative slopes indicate more $\mathrm{H}-$ NS dependent protection at that position and are interpreted to indicated stronger H-NS binding. The Robust- $\mathrm{Z}$ normalized data were used to compare the $\cdot \mathrm{OH}$ footprinting results and the modeled H-NS protection pattern (Figure S7-2). (B) Data in (A) displayed on B-form DNA where red indicates the most protected bases and blue are the least protected bases. 
A

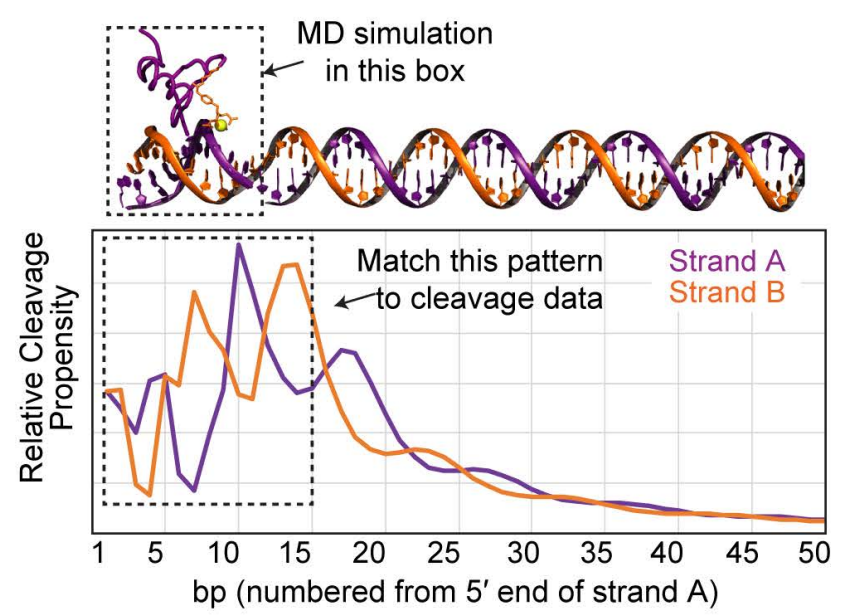

B
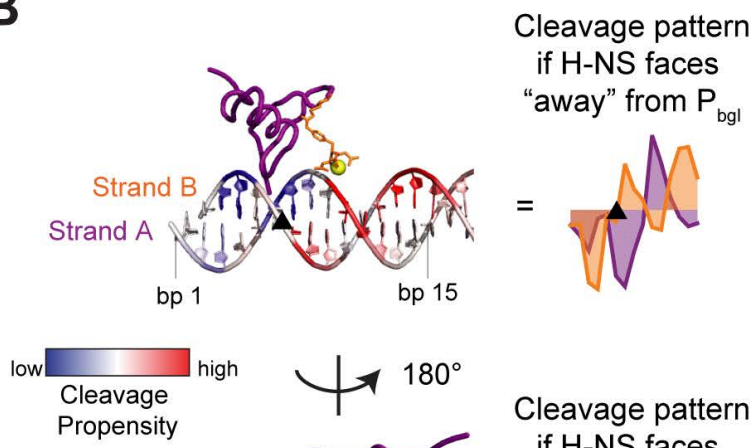

Cleavage pattern if $\mathrm{H}-\mathrm{NS}$ faces "towards" $\mathrm{P}_{\text {bgl }}$

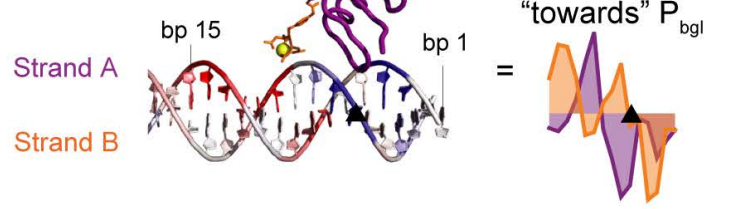

C Tethered Nuclease Cleavage MD Agreement

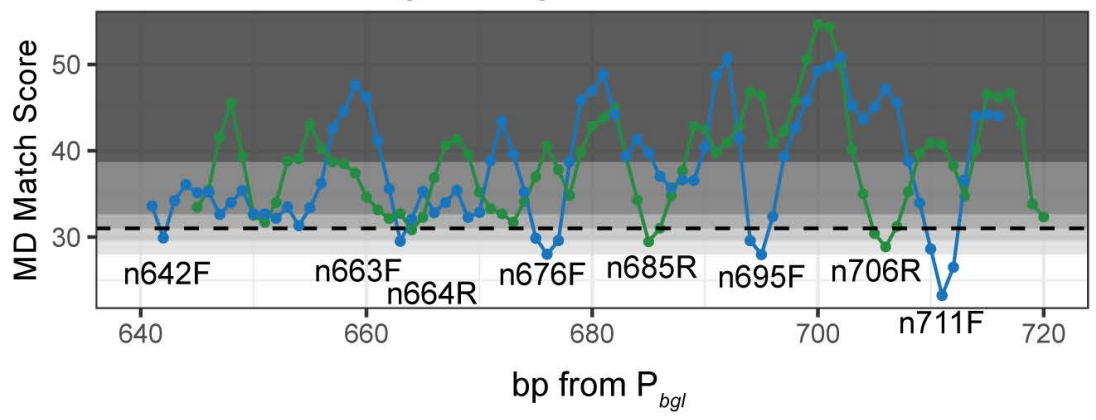

\section{DBD Orientation}

$\rightarrow$ Away from $P_{\text {bgl }}$

$\rightarrow$ Towards $P_{b g l}$

Shuffled Match

Score Percentiles

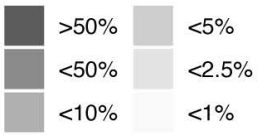

D Final Placement of H-NS DBD in a filament on the DRE fragment

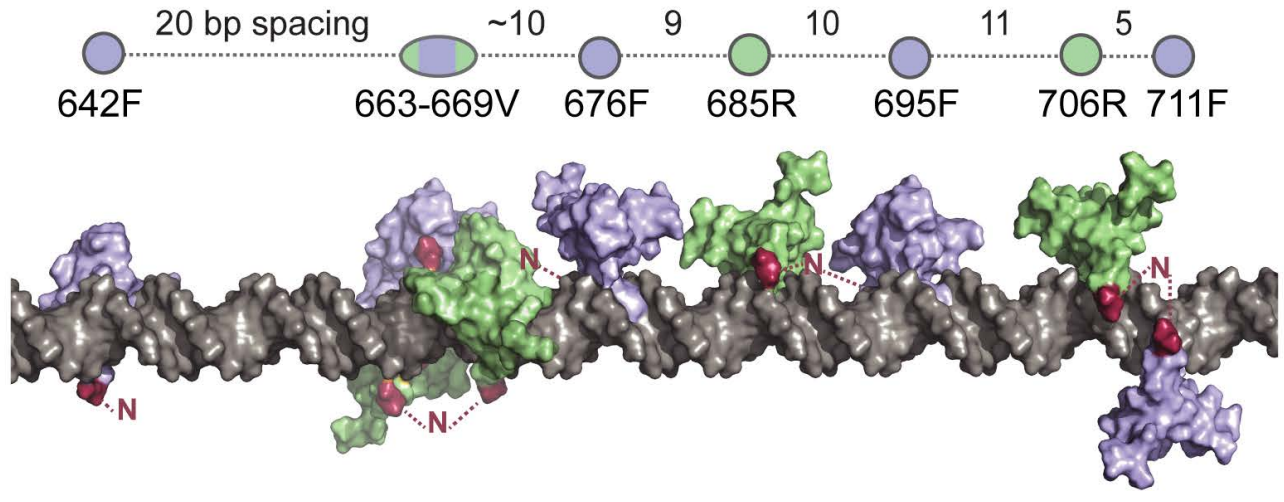

Figure 7. MD simulations of conjugated Fe-EDTA conformational dynamics

(A) MD simulation of Fe'EDTA (orange) conjugated to the H-NS DBD (purple) bound to a 12bp DNA duplex (strand A, purple; strand B, orange). The distance between the $\mathrm{Fe}^{2+}$ and each bp during the simulation was used to estimate the cleavage propensity at each position. Additional DNA (bp 13-50) was included after the MD simulations to estimate cleavage propensities at bp further from the binding site. (B) Overlay of the cleavage propensity on B-form DNA with H-NS bound (PDB 2L93; (Riccardi et al., 2019). Cleavage propensity is on a blue-white-red spectrum where blue is low cleavage propensity and red is high cleavage propensity. Cleavage pattern of H-NS bound in the opposite orientation is also shown. (C) Concordance between expected and observed cleavage patterns as determined by the Manhattan distance (MD Match Score) between 
the pattern of predicted cleavage of the first $15 \mathrm{bp}$ from the MD simulation and the tethered cleavage data of both strands (see Methods). Lower scores indicate greater concordance between the two patterns. The asymmetry of the MD simulation cleavage pattern enabled the determination of DBD orientation with respect to the $\mathrm{P}_{b g l}$ promoter as described in $\mathrm{B}$. Statistical significance was determined by recalculating the Manhattan distance across 1000 shuffled tethered cleavage datasets for the MD cleavage pattern in each orientation. Shaded regions indicate values below the indicated percentiles of MD match scores across all shuffled datasets. The dotted line indicates a MD match score at $<5 \%$ of all match scores determined in the shuffled datasets. The location (bp) and orientation (F or R for forward or reverse) of $8 \mathrm{H}-\mathrm{NS}$ binding sites predicted by TEN-map cleavage are indicated for each DBD. (D) A final model placing 7 H-NS DBDs on the DRE DNA was generated from TEN-map cleavage analysis and $\cdot \mathrm{OH}$ footprinting analysis (Figure S7-2; see Methods). DBDs were either in the forward (slate) or reverse (green). The spacing between each DBD is indicated on the 1D line graph above the $3 \mathrm{D}$ model. The NTD (N) is not placed in our model but would be attached to the DBD at the dark red residues. Associated data: Figure S7-1 and Figure S7-2. 

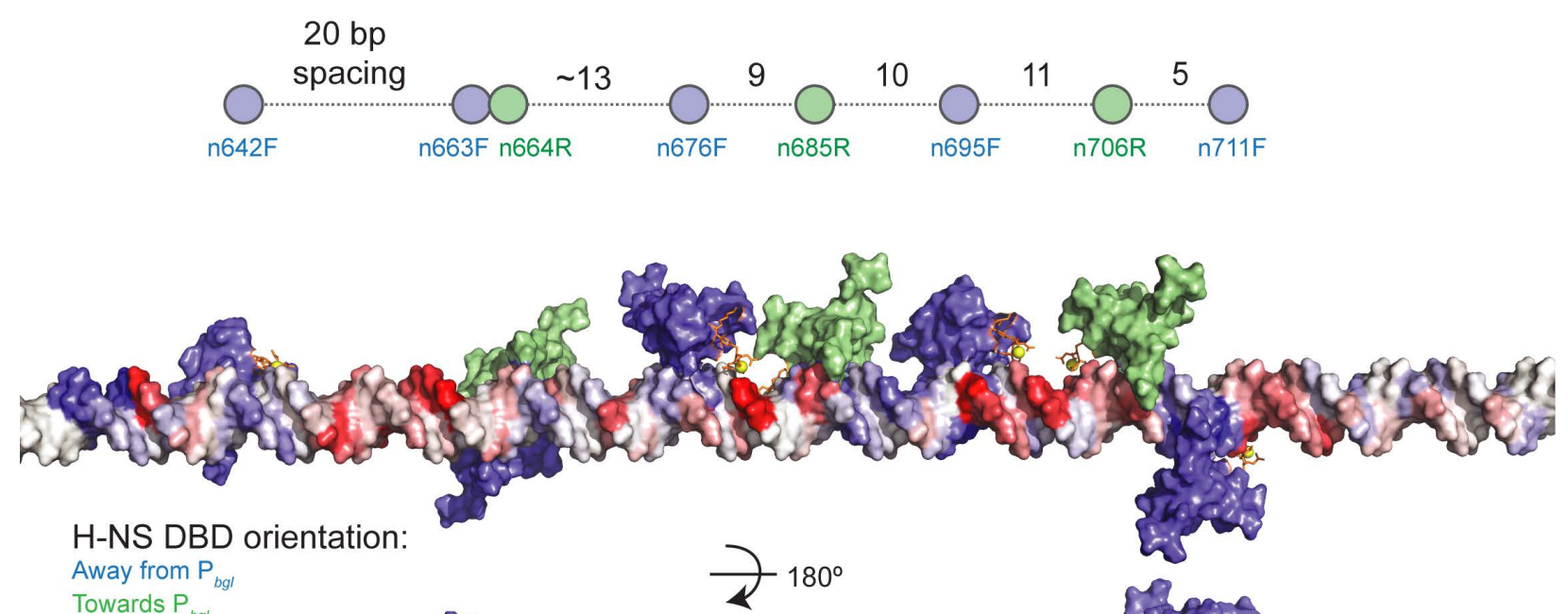

$$
\text { Towards } \mathrm{P}_{b g l}
$$

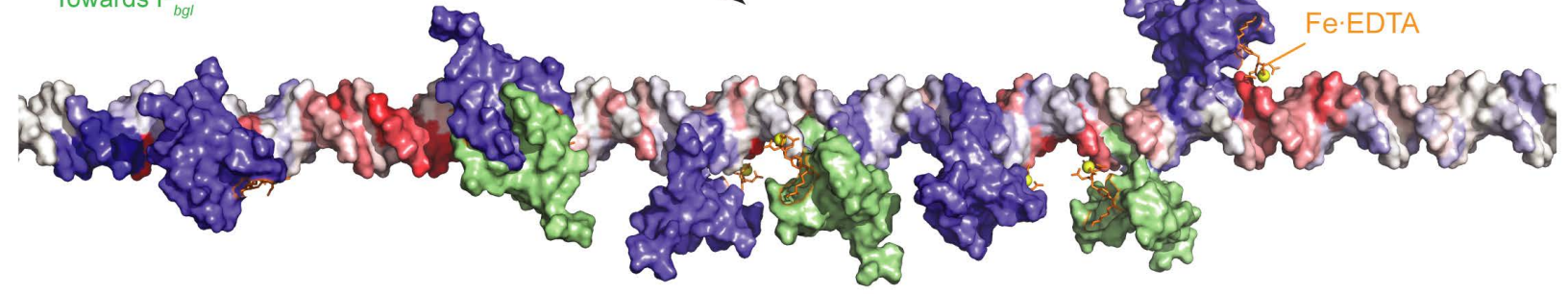

Change in cleavage with [H-NS]

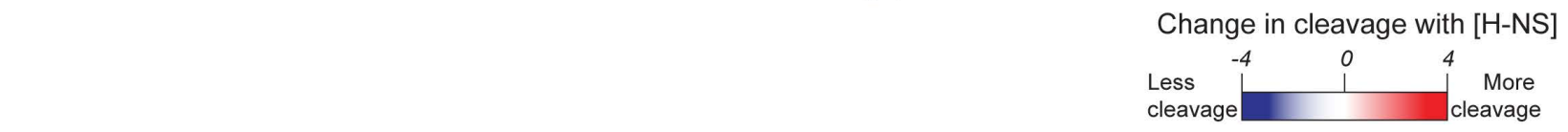

\section{Figure S7-1. Modeling of H-NS DBD location based on match score between TEN-map cleavage pattern and MD simulation.}

The center of the H-NS DBD (slate or green) with the Fe-EDTA tether (orange sticks and yellow sphere, $\mathrm{Fe}^{2+}$ ) was placed on B-form DNA at the position with the best match score in Figure $7 \boldsymbol{C}$. The prediction method ( $\mathrm{n}$ for TEN-map cleavage), position on the DNA (bp from $\mathrm{P}_{b g l}$ ), and the orientation (F or R) are indicated for each DBD. DNA is colored as in Figure S4-5B. Two views of DBD binding model are shown. Spacing between DBD indicated on 1D line plot at the top as in Figure 7. 
A
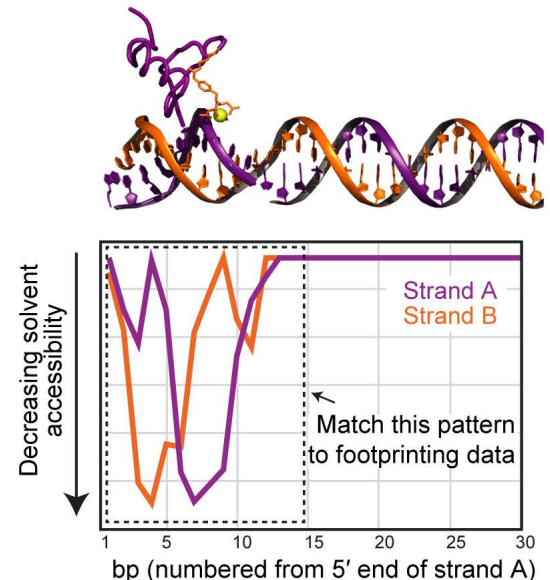

B Cleavage - MD Match Score

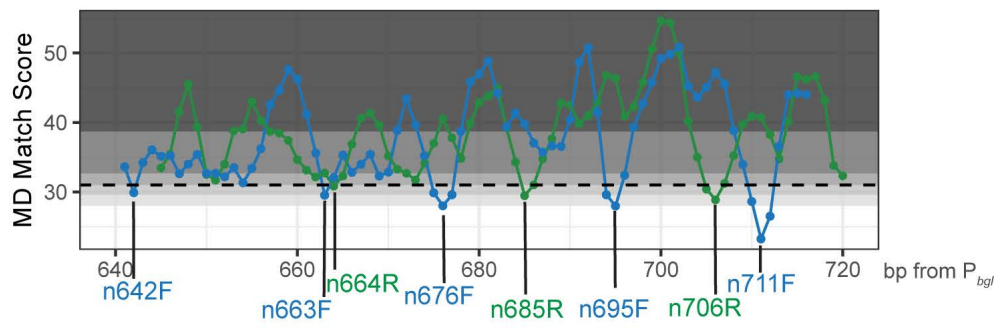

Footprint - MD Match Score

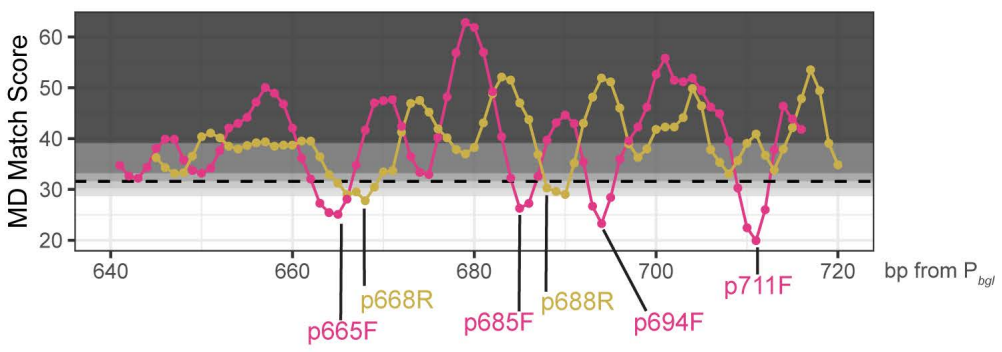

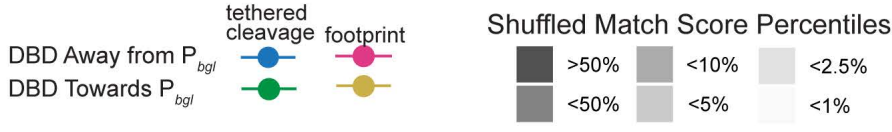

C
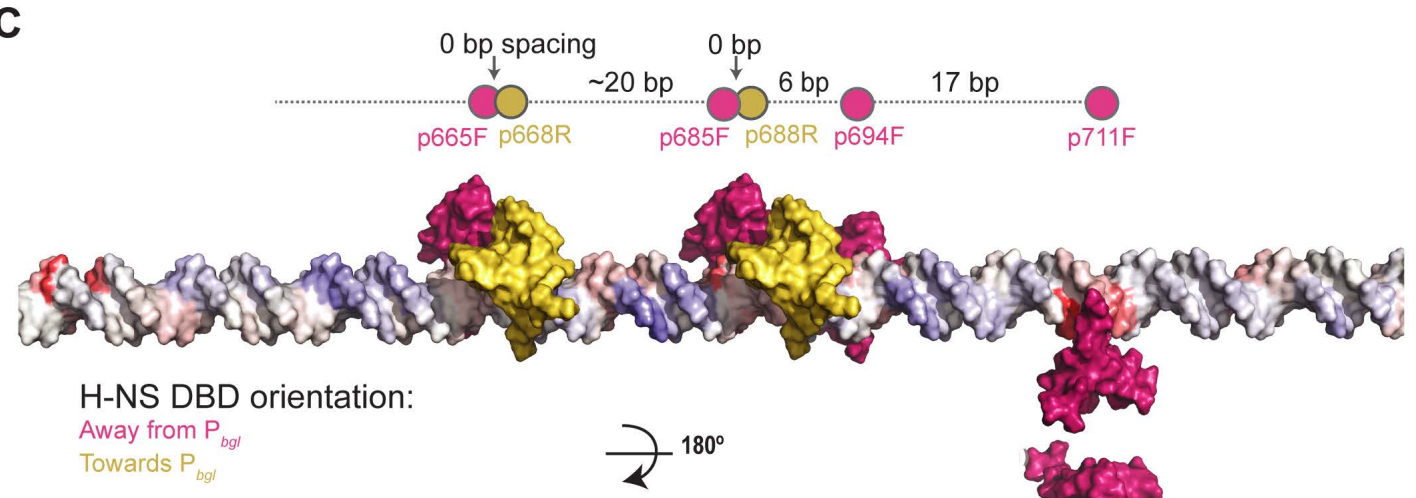

BD orientation:

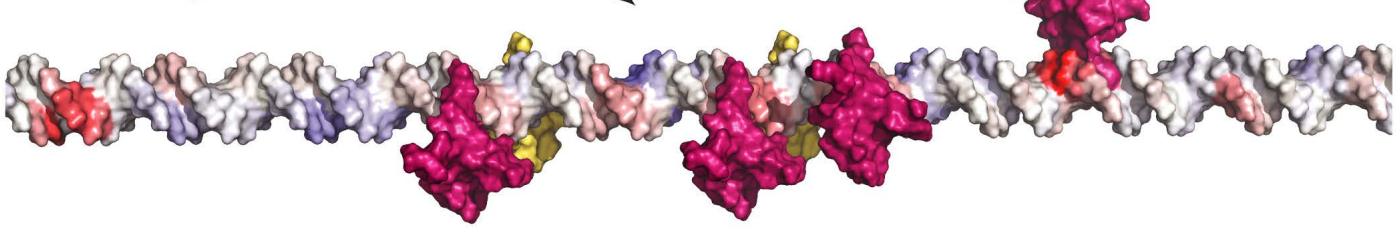

Change in protection with [H-NS]

\section{accessibility patterns.}

Figure S7-2. Modeling of H-NS DBD location based on $\cdot O H$ footprinting and solvent

(A) Solvent accessibility of DNA bound by one H-ND DBD calculated from structure shown at the top. A $15-\mathrm{bp}$ portion of this pattern was compared to the $\cdot \mathrm{OH}$ footprinting pattern (dashed box). (B) Top: repeat of data in Figure $7 \mathrm{C}$ so match scores from $\cdot \mathrm{OH}$ footprinting pattern can be compared to the TEN-map match scores. Bottom: Concordance between expected and observed protection patterns as determined by the Manhattan distance (MD Match Score) between the 
1556

1557

1558

1559

1560

1561

1562

1563

1564

1565

1566

1567

1568

1569

1570

1571

pattern of predicted protection of the first $15 \mathrm{bp}$ from the MD simulation (A) and the $\cdot \mathrm{OH}$ radical footprinting data of both strands (Figure 6; see Methods). Lower scores indicate greater concordance between the two patterns. The asymmetry of the MD simulation cleavage pattern enabled the determination of DBD orientation with respect to the $\mathrm{P}_{b g l}$ promoter with the forward binding cleavage pattern indicating "Away from $\mathrm{P}_{b g}$ " (pink trace) and the reverse binding cleavage indicating "Towards $\mathrm{P}_{b g l}$ " (golden trace). Statistical significance was determined by recalculating the Manhattan distance across 1000 shuffled $\cdot \mathrm{OH}$ footprinting datasets for the MD protection pattern in each orientation. Shaded regions indicate values below the indicated percentiles of MD match scores across all shuffled datasets. The dotted line indicates a MD match score at $<5 \%$ of all match scores determined in the shuffled datasets. (C) The center of the H-NS DBD (gold or pink) was placed on B-form DNA at the position with the best match score in (B). The prediction method ( $\mathrm{p}$ for footprint), position on the DNA (bp from $\mathrm{P}_{b g l}$ ), and the orientation (F or R) are indicated for each DBD. Spacing between DBD indicated on 1D line plot at the top as in Figure 7. Note that there is overlap between the two DBDs at 665 and 668 and the two DBDs at 685 and 688, suggesting that only one H-NS is bound in either of those $3 \mathrm{bp}$ regions. DNA is colored as in Figure S6-2. 
A
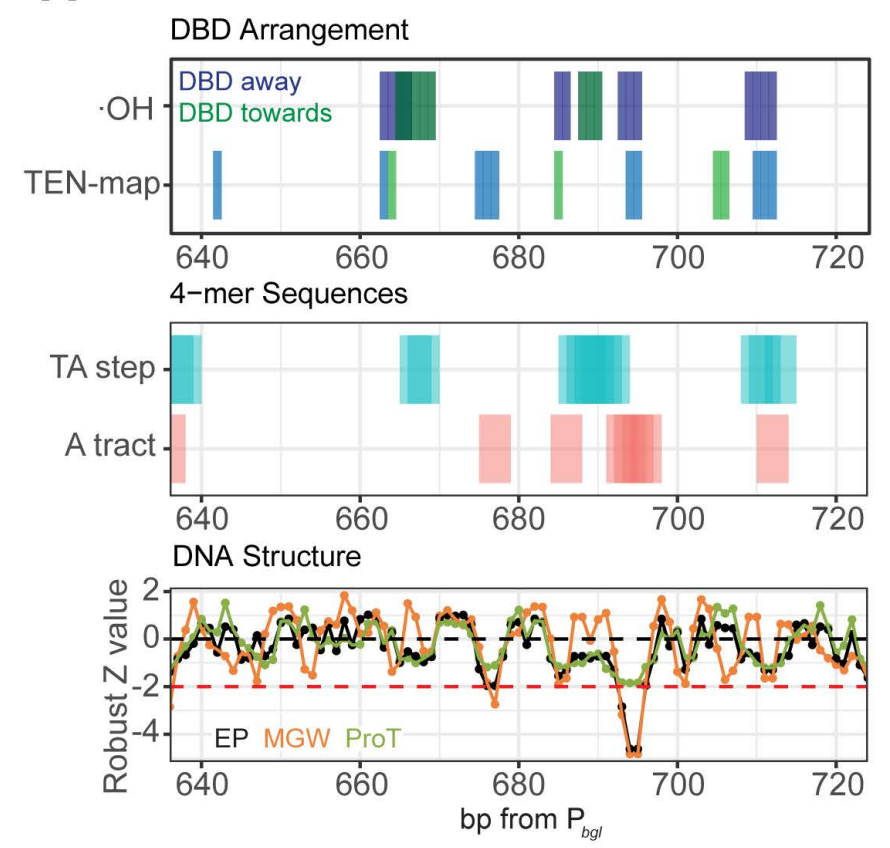

D

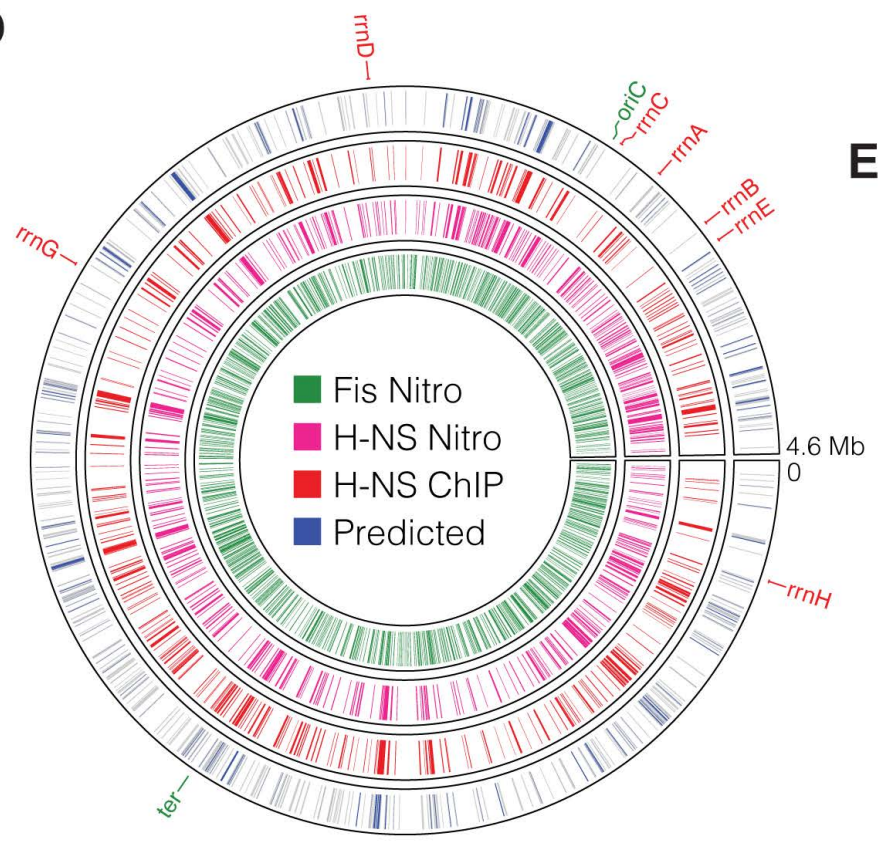

B

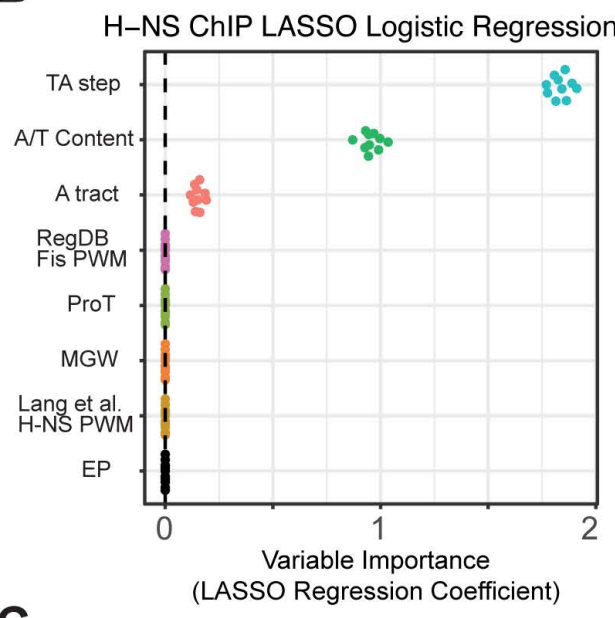

C

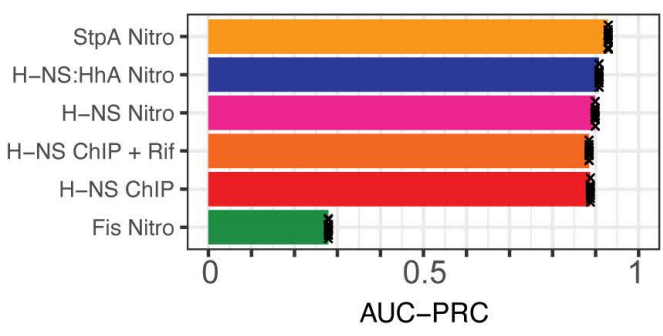

E

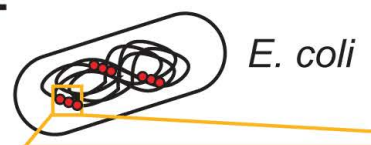

$\mathrm{H}-\mathrm{NS}$ filament locations determined by TA steps, A/T content, \& A-tracts. DBD position \& orientation additionally set by narrow MGW \& negative EP :

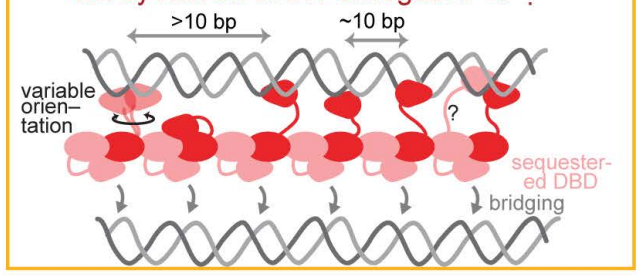

Figure 8. DNA sequence dictates H-NS DBD arrangement within filaments and H-NS filament location genome-wide.

(A) Summary of in vitro analysis of a single H-NS filament and association with DNA sequence and sequence-based structural features. (Top) Regions of high confidence DBD binding and orientation determined from concordance between predicted and observed TEN-map patterns (see Figure 7) as well as predicted and observed DNA protection under $\cdot \mathrm{OH}$ radical footprinting (see Figure S7-2). Each bar indicates regions where match scores between expected and observed patterns are below $95 \%$ of match scores obtained with 1000 shuffled datasets with each 
orientation of the DBD (see Methods). Blue bars indicate a DBD orientation away from $\mathrm{P}_{b g l}$ whereas green bars indicate a DBD orientation towards $\mathrm{P}_{b g l}$. (Middle) Location of 4mers consistent with a TA step or an A-tract (see Methods for definitions) within the DRE sequence. (Bottom) Overlay of predicted minor groove width (MGW, orange), propeller twist (ProT, green), and electrostatic potential in the minor groove (EP, black) across the DRE sequence. The values were Robust- $Z$ scaled and centered using the genome-wide median and MAD (see Methods). The black dotted line indicates the genome-wide median whereas the red dotted line indicates the -2 cutoff used to determine regions with substantially lower values for that feature. (B) Determination of a minimal set of features to predict regions of H-NS binding across the genome. Final coefficients from a LASSO logistic regression model trained on the H-NS ChIP data using the bound status of $1000 \mathrm{bp}$ windows tiled every $250 \mathrm{bp}$ across the genome are displayed with each point representing values determined from one of ten trained models using 10 -fold cross validation with stratified sampling (see Methods). (C) Performance of models trained on predicting bound regions for each genome-wide dataset (from panel B). Each bar displays the average area under a precision recall curve (AUC-PRC) from the 10 replicates of models trained on stratified subsampled data (x indicates each replicate). (D) Circular plot (Gu et al., 2014) displaying the predicted genome-wide H-NS binding sites as determined by a LASSO logistic regression model trained on the full H-NS ChIP-seq data set and only considering windows with a predicted probability $>0.95$ (blue) or between 0.5 and 0.95 (black) of being bound by H-NS as bound regions. For comparison, MOSAiCS determined peaks are shown for H-NS ChIP (red), H-NS Nitro-seq (pink), and Fis Nitro-seq (green). (E) DNA sequence and structure determine global and local H-NS filament arrangement. In E. coli, H-NS filaments form genome-wide at sequences with TA steps, A-tracts, and are AT-rich. Within each filament, most DBDs adopt an arrangement with $\sim 10$ bp spacing between DBDs; however, some DBDs are more flexible and adopt different orientations or bind with small variations in the $\sim 10$ bp spacing along the DNA. The arrangement of H-NS coincides with DNA shape and sequence motif parameters. Our results indicate the same arrangement of the DBD in both bridged or linear filaments, which is best explained by the hemi-sequestration model in which one DBD in an HNS dimer is sequestered by interactions with the NTD in a linear filament but is able to bind 
A
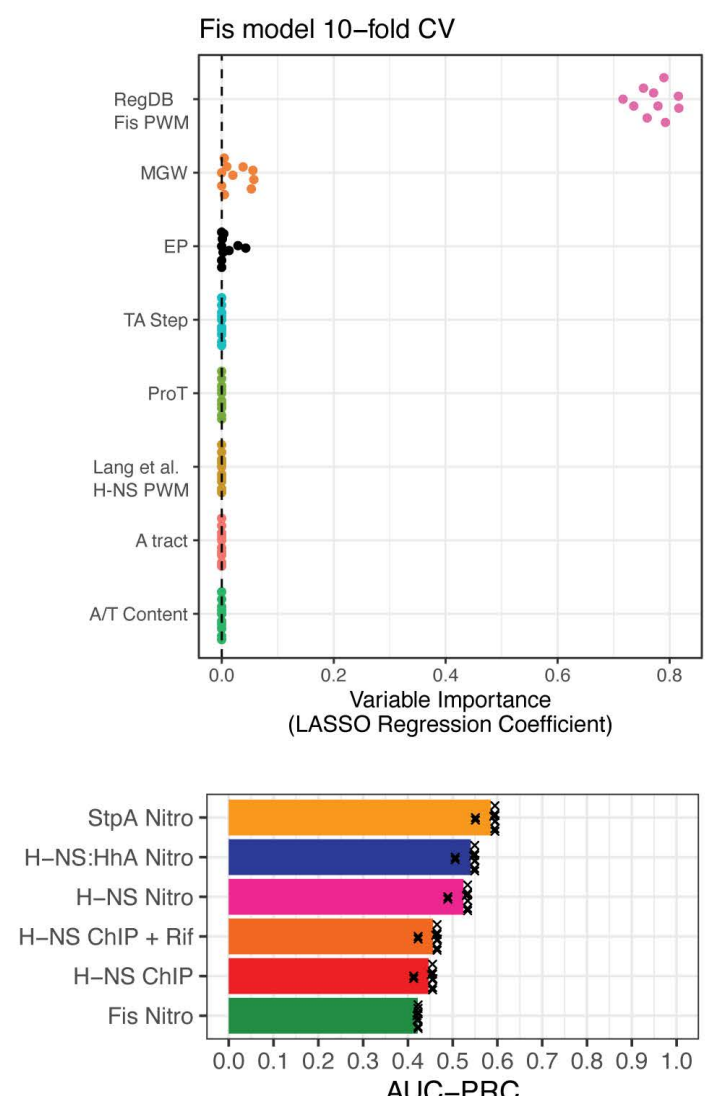

B
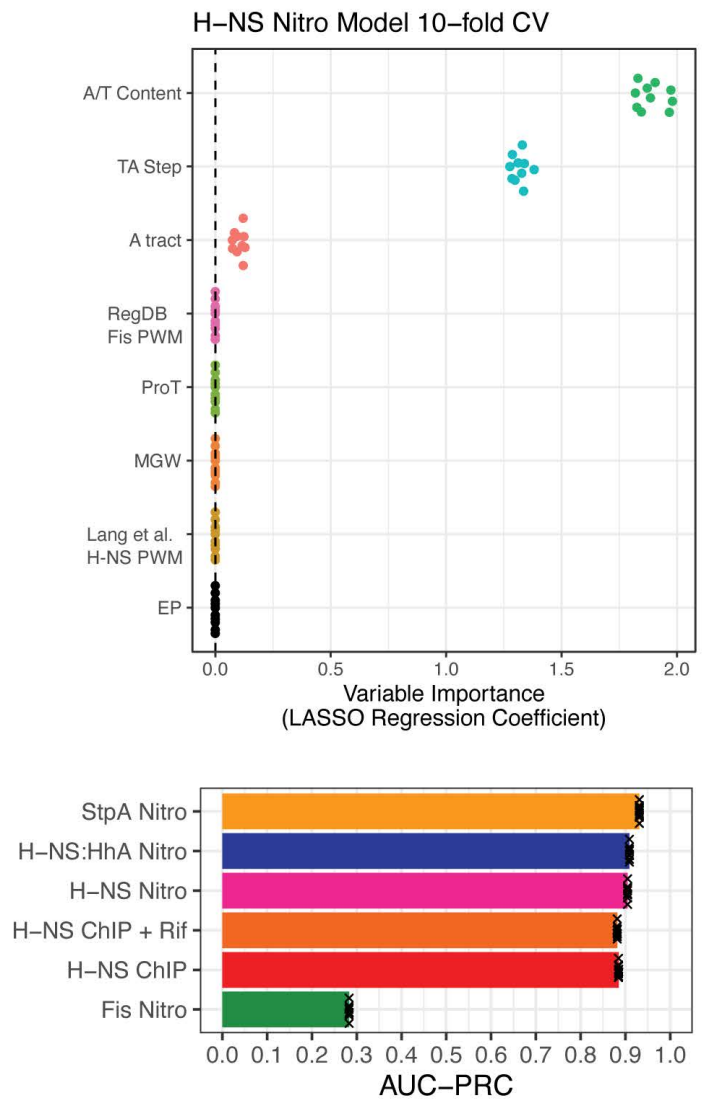

1612 Figure S8-1. LASSO analysis based on H-NS Nitro-seq data also predicts in vivo H-NS 1613 binding pattern, but Fis does not.

1614 (A) Determination of a minimal set of features to predict regions of H-NS binding across the genome using LASSO model trained on the Fis Nitro-seq data (see Methods). Performance of model on predicting bound regions in various genome-wide data sets. Each bar displays the average area under a precision recall curve (AUC-PRC) from the 10 replicates of models trained on stratified subsampled data (x indicates each replicate). (B) As in A, but LASSO model trained on H-NS Nitro-seq data used to predict binding throughout the genome. 


\section{REFERENCES}

1622 Ali, S.S., Whitney, J.C., Stevenson, J., Robinson, H., Howell, P.L., and Navarre, W.W. 2013.

1623 Structural insights into the regulation of foreign genes in Salmonella by the Hha/H-NS

1624 complex. J Biol Chem 288: 13356-13369. doi: 10.1074/jbc.M113.455378.

1625 Amit, R., Oppenheim, A.B., and Stavans, J. 2003. Increased bending rigidity of single DNA molecules by H-NS, a temperature and osmolarity sensor. Biophys. J. 84: 2467-2473. doi:

Andrews, S. (2010). FastQC: a quality control tool for high throughput sequence data. . 10.1016/S0006-3495(03)75051-6.

Arold, S.T., Leonard, P.G., Parkinson, G.N., and Ladbury, J.E. 2010. H-NS forms a superhelical protein scaffold for DNA condensation. PNAS 107: 15728-15732. doi:

1633

1634

1635 10.1073/pnas. 1006966107.

Baba, T., Ara, T., Hasegawa, M., Takai, Y., Okumura, Y., Baba, M., Datsenko, K.A., Tomita, M., Wanner, B.L., and Mori, H. 2006. Construction of Escherichia coli K-12 in-frame, single-gene knockout mutants: the Keio collection. Mol Syst Biol 2: 2006 0008. doi: 10.1038/msb4100050.

Badaut, C., Williams, R., Arluison, V., Bouffartigues, E., Robert, B., Buc, H., and Rimsky, S. 2002. The Degree of Oligomerization of the H-NS Nucleoid Structuring Protein Is Related to Specific Binding to DNA. J. Biol. Chem. 277: 41657-41666. doi: 10.1074/jbc.M206037200.

Balasubramanian, B., Pogozelski, W.K., and Tullis, T.D. 1998. DNA strand breaking by the hydroxyl radical is governed by the accessible surface area of the hydrogen atoms of the DNA backbone. Proc. Natl. Acad. Sci. USA 95: 9738-9743. doi: 10.1073/pnas.95.17.9738.

Banos, R.C., Vivero, A., Aznar, S., Garcia, J., Pons, M., Madrid, C., and Juarez, A. 2009. Differential regulation of horizontally acquired and core genome genes by the bacterial modulator H-NS. PLoS Genet. 5: e1000513. doi: 10.1371/journal.pgen.1000513. 
1645 Best, R.B., Zhu, X., Shim, J., Lopes, P.E.M., Mittal, J., Feig, M., and MacKerell, A.D. 2012.

1646 Optimization of the Additive CHARMM All-Atom Protein Force Field Targeting Improved

1647 Sampling of the Backbone phi, psi and Side-Chain chi(1) and chi(2) Dihedral Angles. Journal

1648 of Chemical Theory and Computation 8: 3257-3273. doi: 10.1021/ct300400x.

1649 Bolger, A.M., Lohse, M., and Usadel, B. 2014. Trimmomatic: a flexible trimmer for Illumina

1650 sequence data. Bioinformatics 30: 2114-2120. doi: 10.1093/bioinformatics/btu170 \%J

1651 Bioinformatics.

1652 Boudreau, B.A., Hron, D.R., Qin, L., van der Valk, R.A., Kotlajich, M.V., Dame, R.T., and

1653 Landick, R. 2018. StpA and Hha stimulate pausing by RNA polymerase by promoting DNA-

1654 DNA bridging of H-NS filaments. Nucleic acids research 46: 5525-5546. doi:

$1655 \quad 10.1093 /$ nar/gky265.

1656 Bouffartigues, E., Buckle, M., Badaut, C., Travers, A., and Rimsky, S. 2007. H-NS cooperative 1657 binding to high-affinity sites in a regulatory element results in transcriptional silencing. Nat. $1658 \quad$ Struct. Mol. Biol. 14: 441-448. doi: 10.1038/nsmb1233.

1659 Brooks, B.R., Brooks, C.L., Mackerell, A.D., Nilsson, L., Petrella, R.J., Roux, B., Won, Y., 1660 Archontis, G., Bartels, C., Boresch, S., et al. 2009. CHARMM: The Biomolecular Simulation 1661 Program. J. Comput. Chem. 30: 1545-1614. doi: 10.1002/Jcc.21287.

1662 Burkhoff, A.M., and Tullis, T.D. 1988. Structural details of an adenine tract that does not cause 1663 DNA to bend. Nature 331: 455-457. doi: 10.1038/331455a0.

1664 Bürkner, P.-C. 2017. brms: An R Package for Bayesian Multilevel Models Using Stan. Journal of $1665 \quad$ Statistical Software 80 doi: 10.18637/jss.v080.i01.

1666 Campbell, E.A., Korzheva, N., Mustaev, A., Murakami, K., Nair, S., Goldfarb, A., and Darst, S.A. 1667 2001. Structural mechanism for rifampicin inhibition of bacterial RNA polymerase. Cell 104: 1668 901-912. doi: 10.1016/s0092-8674(01)00286-0. 
1669 Chandraprakash, D., and Seshasayee, A.S.N. 2014. Inhibition of factor-dependent transcription

1670 termination in Escherichia coli might relieve xenogene silencing by abrogating H-NS-DNA

1671 interactions in vivo. J. Biosci. (Bangalore) 39: 53-61. doi: 10.1007/s12038-014-9413-4.

1672 Cheal, S.M., Ng, M., Barrios, B., Miao, Z., Kalani, A.K., and Meares, C.F. 2009. Mapping

1673 protein-protein interactions by localized oxidation: consequences of the reach of hydroxyl

1674 radical. Biochemistry 48: 4577-4586. doi: 10.1021/bi900273j.

1675 Chiu, T.-P., Rao, S., Mann, R.S., Honig, B., and Rohs, R. 2017. Genome-wide prediction of 1676 minor-groove electrostatic potential enables biophysical modeling of protein-DNA binding.

1677 Nucleic Acids Res. 45: 12565-12576. doi: 10.1093/nar/gkx915.

1678 Chiu, T.P., Comoglio, F., Zhou, T., Yang, L., Paro, R., and Rohs, R. 2016. DNAshapeR: an

1679 R/Bioconductor package for DNA shape prediction and feature encoding. Bioinformatics 32:

1680 1211-1213. doi: 10.1093/bioinformatics/btv735.

1681 Cho, B.K., Knight, E.M., Barrett, C.L., and Palsson, B.O. 2008. Genome-wide analysis of Fis

1682 binding in Escherichia coli indicates a causative role for A-/AT-tracts. Genome Res. 18: 900-

1683 910. doi: 10.1101/gr.070276.107.

1684 Chung, D., Zhang, Q., and Keles, S. (2014). MOSAiCS-HMM: A model-based approach for 1685 detecting regions of histone modifications from ChIP-seq data. In Statistical Analysis of Next 1686 Generation Sequencing Data, S. Data, and D. Nettleton, eds. (Springer).

1687 Cook, P.R., and Marenduzzo, D. 2018. Transcription-driven genome organization: a model for 1688 chromosome structure and the regulation of gene expression tested through simulations.

1689 Nucleic Acids Res. 46: 9895-9906. doi: 10.1093/nar/gky763.

1690 Dame, R.T., Noom, M.C., and Wuite, G.J. 2006. Bacterial chromatin organization by H-NS

1691 protein unravelled using dual DNA manipulation. Nature 444: 387-390. doi:

$1692 \quad 10.1038 /$ nature05283. 
1693 Dame, R.T., Rashid, F.M., and Grainger, D.C. 2020. Chromosome organization in bacteria:

1694 mechanistic insights into genome structure and function. Nat. Rev. Genet. doi:

$1695 \quad 10.1038 / \mathrm{s} 41576-019-0185-4$.

1696 Das, R., Laederach, A., Pearlman, S.M., Herschlag, D., and Altman, R.B. 2005. SAFA: semi1697 automated footprinting analysis software for high-throughput quantification of nucleic acid 1698 footprinting experiments. RNA 11: 344-354. doi: 10.1261/rna.7214405.

1699 Davis, J., and Goadrich, M. (2006). The relationship between Precision-Recall and ROC curves.

1700 In Proceedings of the 23rd international conference on Machine learning (Pittsburgh,

1701 Pennsylvania, USA, Association for Computing Machinery), pp. 233-

$1702 \quad 240^{\wedge} 10.1145 / 1143844.1143874$.

1703 Dole, S., Nagarajavel, V., and Schnetz, K. 2004. The histone-like nucleoid structuring protein H-

1704 NS represses the Escherichia coli bgl operon downstream of the promoter. Mol Microbiol 52:

1705 589-600. doi: 10.1111/j.1365-2958.2004.04001.x.

1706 Eastman, P., Swails, J., Chodera, J.D., McGibbon, R.T., Zhao, Y.T., Beauchamp, K.A., Wang,

1707 L.P., Simmonett, A.C., Harrigan, M.P., Stern, C.D., et al. 2017. OpenMM 7: Rapid

1708 development of high performance algorithms for molecular dynamics. PLoS Comp. Biol. 13:

1709 17. doi: 10.1371/journal.pcbi.1005659.

1710 Favorov, A., Mularoni, L., Cope, L.M., Medvedeva, Y., Mironov, A.A., Makeev, V.J., and

1711 Wheelan, S.J. 2012. Exploring Massive, Genome Scale Datasets with the GenometriCorr

1712 Package. PLoS Comp. Biol. 8: e1002529. doi: 10.1371/journal.pcbi.1002529.

1713 Friedman, J.H., Hastie, T., and Tibshirani, R. 2010. Regularization Paths for Generalized Linear

1714 Models via Coordinate Descent. 2010 33: 22 \%J Journal of Statistical Software. doi:

$1715 \quad 10.18637 /$ jss.v033.i01. 
1716 Fukui, N., Oshima, T., Ueda, T., Ogasawara, N., and Tobe, T. 2016. Gene Activation through the

1717 Modulation of Nucleoid Structures by a Horizontally Transferred Regulator, Pch, in

1718 Enterohemorrhagic Escherichia coli. PLoS One 11: e0149718. doi:

1719 10.1371/journal.pone.0149718.

1720 Gao, Y., Foo, Y.H., Winardhi, R.S., Tang, Q., Yan, J., and Kenney, L.J. 2017. Charged residues

1721 in the H-NS linker drive DNA binding and gene silencing in single cells. Proc Natl Acad Sci

1722 USA 114: 12560-12565. doi: 10.1073/pnas.1716721114.

1723 Gawade, P., Gunjal, G., Sharma, A., and Ghosh, P. 2020. Reconstruction of transcriptional

1724 regulatory networks of Fis and H-NS in Escherichia coli from genome-wide data analysis.

1725 Genomics 112: 1264-1272. doi: https://doi.org/10.1016/j.ygeno.2019.07.013.

1726 Ghosh, I.N., and Landick, R. 2016. OptSSeq: High-Throughput Sequencing Readout of Growth

1727 Enrichment Defines Optimal Gene Expression Elements for Homoethanologenesis. ACS Synth

1728 Biol 5: 1519-1534. doi: 10.1021/acssynbio.6b00121.

1729 Gordon, B.R., Li, Y., Cote, A., Weirauch, M.T., Ding, P., Hughes, T.R., Navarre, W.W., Xia, B., 1730 and Liu, J. 2011. Structural basis for recognition of AT-rich DNA by unrelated xenogeneic 1731 silencing proteins. Proc Natl Acad Sci USA 108: 10690-10695. doi: 10.1073/pnas.1102544108.

1732 Grainger, D.C. 2016. Structure and function of bacterial H-NS protein. Biochem. Soc. Trans. 44:

1733 1561-1569. doi: 10.1042/BST20160190.

1734 Grainger, D.C., Goldberg, M.D., Lee, D.J., and Busby, S.J. 2008. Selective repression by Fis and 1735 H-NS at the Escherichia coli dps promoter. Mol Microbiol 68: 1366-1377. doi: 10.1111/j.1365$1736 \quad 2958.2008 .06253 . x$.

1737 Grainger, D.C., Hurd, D., Goldberg, M.D., and Busby, S.J. 2006. Association of nucleoid proteins 1738 with coding and non-coding segments of the Escherichia coli genome. Nucleic acids research 1739 34: 4642-4652. doi: 10.1093/nar/gk1542. 
1740 Grant, C.E., Bailey, T.L., and Noble, W.S. 2011. FIMO: scanning for occurrences of a given

1741 motif. Bioinformatics 27: 1017-1018. doi: 10.1093/bioinformatics/btr064.

1742 Grau, J., Grosse, I., and Keilwagen, J. 2015. PRROC: computing and visualizing precision-recall

1743 and receiver operating characteristic curves in R. Bioinformatics 31: 2595-2597. doi:

1744 10.1093/bioinformatics/btv153 \% J Bioinformatics.

1745 Greiner, D.P., Miyake, R., Moran, J.K., Jones, A.D., Negishi, T., Ishihama, A., and Meares, C.F.

1746 1997. Synthesis of the protein cutting reagent Iron $(S)-1-(p-$

1747 Bromoacetamidobenzyl)ethylenediaminetetraacetate and conjugation to cysteine side chains.

1748 Bioconj. Chem. 8: 44-48. doi: 10.1021/bc9600731.

1749 Gu, Z., Gu, L., Eils, R., Schlesner, M., and Brors, B. 2014. circlize implements and enhances

1750 circular visualization in R. Bioinformatics 30: 2811-2812. doi: 10.1093/bioinformatics/btu393

$1751 \% \mathrm{~J}$ Bioinformatics.

1752 Gulvady, R., Gao, Y., Kenney, L.J., and Yan, J. 2018. A single molecule analysis of H-NS

1753 uncouples DNA binding affinity from DNA specificity. Nucleic acids research 46: 10216-

1754 10224. doi: 10.1093/nar/gky826.

1755 Hampel, F.R. 1974. The Influence Curve and its Role in Robust Estimation. Journal of the

1756 American Statistical Association 69: 383-393. doi: 10.1080/01621459.1974.10482962.

1757 Hart, K., Foloppe, N., Baker, C.M., Denning, E.J., Nilsson, L., and MacKerell, A.D. 2012.

1758 Optimization of the CHARMM Additive Force Field for DNA: Improved Treatment of the

1759 BI/BII Conformational Equilibrium. Journal of Chemical Theory and Computation 8: 348-362.

1760 doi: $10.1021 /$ ct200723y.

1761 Herring, C.D., Raffaelle, M., Allen, T.E., Kanin, E.I., Landick, R., Ansari, A.Z., and Palsson,

1762 B.O. 2005. Immobilization of Escherichia coli RNA polymerase and location of binding sites 
by use of chromatin immunoprecipitation and microarrays. J Bacteriol 187: 6166-6174. doi:

1765

1766

1767

1768

1769

1770

1771

1772

1773

1774

1775

1776

1777

1778

1779

1780

1781

1782

1783

1784

1785

1786

Japaridze, A., Renevey, S., Sobetzko, P., Stoliar, L., Nasser, W., Dietler, G., and Muskhelishvili, G. 2017. Spatial organization of DNA sequences directs the assembly of bacterial chromatin by a nucleoid-associated protein. J Biol Chem 292: 7607-7618. doi: 10.1074/jbc.M117.780239.

Kahramanoglou, C., Seshasayee, A.S., Prieto, A.I., Ibberson, D., Schmidt, S., Zimmermann, J., Benes, V., Fraser, G.M., and Luscombe, N.M. 2011. Direct and indirect effects of H-NS and Fis on global gene expression control in Escherichia coli. Nucleic Acids Res 39: 2073-2091. doi: $10.1093 /$ nar/gkq934.

Kim, D., Hong, J.S., Qiu, Y., Nagarajan, H., Seo, J.H., Cho, B.K., Tsai, S.F., and Palsson, B.O. 2012. Comparative analysis of regulatory elements between Escherichia coli and Klebsiella pneumoniae by genome-wide transcription start site profiling. PLoS Genet. 8: e1002867. doi: 10.1371/journal.pgen.1002867.

Kotlajich, M.V., Hron, D.R., Boudreau, B.A., Sun, Z., Lyubchenko, Y.L., and Landick, R. 2015. Bridged filaments of histone-like nucleoid structuring protein pause RNA polymerase and aid termination in bacteria. Elife 4 doi: 10.7554/eLife.04970.

Krietenstein, N., Wal, M., Watanabe, S., Park, B., Peterson, C.L., Pugh, B.F., and Korber, P. 2016. Genomic Nucleosome Organization Reconstituted with Pure Proteins. Cell 167: 709-721 e712. doi: 10.1016/j.cell.2016.09.045.

Kuan, P.F., Chung, D., Pan, G., Thomson, J.A., Stewart, R., and Keles, S. 2011. A Statistical Framework for the Analysis of ChIP-Seq Data. J Am Stat Assoc 106: 891-903. doi: 10.1198/jasa.2011.ap09706.

Lang, B., Blot, N., Bouffartigues, E., Buckle, M., Geertz, M., Gualerzi, C.O., Mavathur, R., Muskhelishvili, G., Pon, C.L., Rimsky, S., et al. 2007. High-affinity DNA binding sites for H- 
1787 NS provide a molecular basis for selective silencing within proteobacterial genomes. Nucleic

1788 Acids Res 35: 6330-6337. doi: 10.1093/nar/gkm712.

1789 Langmead, B., and Salzberg, S.L. 2012. Fast gapped-read alignment with Bowtie 2. Nat. Methods 1790 9: 357-359. doi: 10.1038/nmeth.1923.

1791 Leonard, P.G., Ono, S., Gor, J., Perkins, S.J., and Ladbury, J.E. 2009. Investigation of the self1792 association and hetero-association interactions of H-NS and StpA from Enterobacteria. Mol Microbiol 73: 165-179. doi: 10.1111/j.1365-2958.2009.06754.x.

1794 Li, H., Handsaker, B., Wysoker, A., Fennell, T., Ruan, J., Homer, N., Marth, G., Abecasis, G., 1795 Durbin, R., and Subgroup, G.P.D.P. 2009. The Sequence Alignment/Map format and 1796 SAMtools. Bioinformatics 25: 2078-2079. doi: 10.1093/bioinformatics/btp352 \%J 1797 Bioinformatics.

1798

1799

1800

1801 1802 1803

Liao, Q.H., Pabis, A., Strodel, B., and Kamerlin, S.C.L. 2017. Extending the Nonbonded Cationic Dummy Model to Account for Ion-Induced Dipole Interactions. Journal of Physical Chemistry Letters 8: 5408-5414. doi: 10.1021/acs.jpclett.7b02358.

Lim, C.J., Lee, S.Y., Kenney, L.J., and Yan, J. 2012. Nucleoprotein filament formation is the structural basis for bacterial protein H-NS gene silencing. Sci Rep 2: 509. doi:

$$
10.1038 / \text { srep00509. }
$$

Liu, Y., Chen, H., Kenney, L.J., and Yan, J. 2010. A divalent switch drives H-NS/DNA-binding conformations between stiffening and bridging modes. Genes Dev 24: 339-344. doi: $10.1101 / \operatorname{gad} .1883510$.

Lucchini, S., McDermott, P., Thompson, A., and Hinton, J.C. 2009. The H-NS-like protein StpA represses the RpoS (sigma 38) regulon during exponential growth of Salmonella Typhimurium. Mol Microbiol 74: 1169-1186. doi: 10.1111/j.1365-2958.2009.06929.x. 
1810 Lucchini, S., Rowley, G., Goldberg, M.D., Hurd, D., Harrison, M., and Hinton, J.C. 2006. H-NS

1811 mediates the silencing of laterally acquired genes in bacteria. PLoS Pathog 2: e81. doi:

$1812 \quad$ 10.1371/journal.ppat.0020081.

1813 Luger, K., Mader, A.W., Richmond, R.K., Sargent, D.F., and Richmond, T.J. 1997. Crystal

1814 structure of the nucleosome core particle at $2.8 \AA$ resolution. Nature 389: 251-260. doi:

$1815 \quad 10.1038 / 38444$.

1816 Maxam, A.M., and Gilbert, W. 1977. A new method for sequencing DNA. Proc. Natl. Acad. Sci.

1817 USA 74: 560-564. doi: 10.1073/pnas.74.2.560.

1818 Mooney, R.A., Davis, S.E., Peters, J.M., Rowland, J.L., Ansari, A.Z., and Landick, R. 2009.

1819 Regulator trafficking on bacterial transcription units in vivo. Mol Cell 33: 97-108. doi:

$1820 \quad$ 10.1016/j.molcel.2008.12.021.

1821 Muller, C.M., Dobrindt, U., Nagy, G., Emody, L., Uhlin, B.E., and Hacker, J. 2006. Role of 1822 histone-like proteins H-NS and StpA in expression of virulence determinants of uropathogenic 1823 Escherichia coli. J Bacteriol 188: 5428-5438. doi: 10.1128/JB.01956-05.

1824 Myers, K.S., Yan, H., Ong, I.M., Chung, D., Liang, K., Tran, F., Keles, S., Landick, R., and 1825 Kiley, P.J. 2013. Genome-scale analysis of escherichia coli FNR reveals complex features of 1826 transcription factor binding. PLoS Genet. 9: e1003565. doi: 10.1371/journal.pgen.1003565.

1827 Nagarajavel, V., Madhusudan, S., Dole, S., Rahmouni, A.R., and Schnetz, K. 2007. Repression by 1828 binding of H-NS within the transcription unit. J Biol Chem 282: 23622-23630. doi:

$1829 \quad 10.1074 /$ jbc.M702753200.

1830 Navarre, W.W., Porwollik, S., Wang, Y., McClelland, M., Rosen, H., Libby, S.J., and Fang, F.C.

1831 2006. Selective silencing of foreign DNA with low GC content by the H-NS protein in

1832 Salmonella. Science 313: 236-238. doi: 10.1126/science. 1128794. 
1833 Neidhardt, F.C., Bloch, P.L., and Smith, D.F. 1974. Culture Medium for Enterobacteria. 119:

1834 736-747. doi:

1835 Nieto, J.M., Madrid, C., Prenafeta, A., Miquelay, E., Balsalobre, C., Carrascal, M., and Juarez, A.

1836 2000. Expression of the hemolysin operon in Escherichia coli is modulated by a nucleoid-

1837 protein complex that includes the proteins Hha and H-NS. Mol. Genet. Genomics 263: 349-

$1838 \quad$ 358. doi: $10.1007 / \mathrm{s} 004380051178$.

1839 Noom, M.C., Navarre, W.W., Oshima, T., Wuite, G.J., and Dame, R.T. 2007. H-NS promotes

1840 looped domain formation in the bacterial chromosome. Current biology : CB 17: R913-914.

1841 doi: 10.1016/j.cub.2007.09.005.

1842 Owen-Hughes, T., Pavitt, G., Santos, D., Sidebotham, J., Hulton, C., Hinton, J., and Higgins, C.

1843 1992. The chromatin-associated protein H-NS interacts with curved DNA to influence DNA

1844 topology and gene expression. . Cell 71: 255-265. doi: 10.1002/j.1460-2075.1994.tb06256.x.

1845 Papoulas, O. 1996. Rapid Separation of Protein-Bound DNA from Free DNA Using

1846 Nitrocellulose Filters. Curr Protoc Mol Biol: 12.18.11-12.18.19. doi:

$1847 \quad 10.1002 / 0471142727 . m b 1208 s 36$.

1848 Peters, J.M., Mooney, R.A., Grass, J.A., Jessen, E.D., Tran, F., and Landick, R. 2012. Rho and

1849 NusG suppress pervasive antisense transcription in Escherichia coli. Genes Dev 26: 2621-2633.

1850 doi: $10.1101 /$ gad.196741.112.

1851 Quinlan, A.R., and Hall, I.M. 2010. BEDTools: a flexible suite of utilities for comparing genomic 1852 features. Bioinformatics 26: 841-842. doi: 10.1093/bioinformatics/btq033.

1853 Rangarajan, A.A., and Schnetz, K. 2018. Interference of transcription across H-NS binding sites 1854 and repression by H-NS. Mol Microbiol 108: 226-239. doi: 10.1111/mmi.13926. 
1855 Riccardi, E., van Mastbergen, E.C., Navarre, W.W., and Vreede, J. 2019. Predicting the

1856 mechanism and rate of H-NS binding to AT-rich DNA. PLoS Comput Biol 15: e1006845. doi:

$1857 \quad$ 10.1371/journal.pcbi.1006845.

1858 Rousseeuw, P.J., and Croux, C. 1993. Alternatives to the Median Absolute Deviation. Journal of 1859 the American Statistical Association 88: 1273-1283. doi: 10.1080/01621459.1993.10476408.

1860 Sankar, T.S., Neelakanta, G., Sangal, V., Plum, G., Achtman, M., and Schnetz, K. 2009. Fate of 1861 the H-NS-repressed bgl operon in evolution of Escherichia coli. PLoS Genet. 5: e1000405. doi: 1862 10.1371/journal.pgen.1000405.

Santos-Zavaleta, A., Salgado, H., Gama-Castro, S., Sanchez-Perez, M., Gomez-Romero, L., Ledezma-Tejeida, D., Garcia-Sotelo, J.S., Alquicira-Hernandez, K., Muniz-Rascado, L.J., Pena-Loredo, P., et al. 2019. RegulonDB v 10.5: tackling challenges to unify classic and high throughput knowledge of gene regulation in E. coli K-12. Nucleic Acids Res 47: D212-D220. doi: 10.1093/nar/gky1077.

Saxena, S., and Gowrishankar, J. 2011. Modulation of Rho-Dependent Transcription Termination in Escherichia coli by the H-NS Family of Proteins. J. Bacteriol. 193: 3832. doi: 10.1128/JB.00220-11.

Schnetz, K. 1995. Silencing of the Escherichia coli bgl promoter by flanking sequence elements.

1873 Shahul Hameed, U.F., Liao, C., Radhakrishnan, A.K., Huser, F., Aljedani, S.S., Zhao, X., Momin, conformational switch for environment-controlled gene silencing. Nucleic Acids Res 47: 26662680. doi: 10.1093/nar/gky1299.

1877 Shen, B.A., and Landick, R. 2019. Transcription of Bacterial Chromatin. J. Mol. Biol. doi: $1878 \quad$ 10.1016/j.jmb.2019.05.041. 
1879

1880

1881

1882

1883

1884

1885

1886

1887

1888

1889

1890

1891

1892

1893

1894

1895

1896

1897

1898

1899

1900

1901

Singh, S.S., Singh, N., Bonocora, R.P., Fitzgerald, D.M., Wade, J.T., and Grainger, D.C. 2014. Widespread suppression of intragenic transcription initiation by H-NS. Genes Dev 28: 214219. doi: $10.1101 / \operatorname{gad} .234336 .113$.

Sonnenfield, J.M., Burns, C.M., Higgins, C.F., and Hinton, J.C. 2001. The nucleoid-associated protein StpA binds curved DNA, has a greater DNA binding affinity than H-NS and is present in significant levels in hns mutants. Biochimie 83: 243-249. doi: 10.1016/s03009084(01)01232-9.

Srinivasan, R., Chandraprakash, D., Krishnamurthi, R., Singh, P., Scolari, V.F., Krishna, S., and Seshasayee, A.S. 2013. Genomic analysis reveals epistatic silencing of "expensive" genes in Escherichia coli K-12. Mol Biosyst 9: 2021-2033. doi: 10.1039/c3mb70035f.

Stoebel, D.M., Free, A., and Dorman, C.J. 2008. Anti-silencing: overcoming H-NS-mediated repression of transcription in Gram-negative enteric bacteria. 154: 2533-2545. doi: https://doi.org/10.1099/mic.0.2008/020693-0.

Talbert, P.B., Meers, M.P., and Henikoff, S. 2019. Old cogs, new tricks: the evolution of gene expression in a chromatin context. Nat. Rev. Genet. 20: 283-297. doi: 10.1038/s41576-0190105-7.

Thomason, M.K., Bischler, T., Eisenbart, S.K., Forstner, K.U., Zhang, A., Herbig, A., Nieselt, K., Sharma, C.M., and Storz, G. 2015. Global transcriptional start site mapping using differential RNA sequencing reveals novel antisense RNAs in Escherichia coli. J Bacteriol 197: 18-28. doi: 10.1128/JB.02096-14.

Tupper, A.E., Owen-Hughes, T.A., Ussery, D.W., Santos, D.S., Ferguson, D.J.P., Sidebotham, J.M., Hinton, J.C., and Higgins, C.F. 1994. The chromatin-associated protein H-NS alters DNA topology in vitro. EMBO J 13: 258-268. doi: 10.1002/j.1460-2075.1994.tb06256.xs. 
1902 Ueda, T., Takahashi, H., Uyar, E., Ishikawa, S., Ogasawara, N., and Oshima, T. 2013. Functions

1903 of the Hha and YdgT proteins in transcriptional silencing by the nucleoid proteins, H-NS and

1904 StpA, in Escherichia coli. DNA Res. 20: 263-271. doi: 10.1093/dnares/dst008.

1905 Ulissi, U., Fabbretti, A., Sette, M., Giuliodori, A.M., and Spurio, R. 2014. Time-resolved 1906 assembly of a nucleoprotein complex between Shigella flexneri virF promoter and its

1907 transcriptional repressor H-NS. Nucleic Acids Res 42: 13039-13050. doi: 10.1093/nar/gku1052.

1908 Uyar, E., Kurokawa, K., Yoshimura, M., Ishikawa, S., Ogasawara, N., and Oshima, T. 2009.

1909 Differential binding profiles of StpA in wild-type and h-ns mutant cells: a comparative analysis

1910 of cooperative partners by chromatin immunoprecipitation-microarray analysis. J Bacteriol

1911 191: 2388-2391. doi: 10.1128/JB.01594-08.

1912 van der Valk, R.A., Qin, L., Moolenaar, G.F., and Dame, R.T. (2018). Quantitative Determination 1913 of DNA Bridging Efficiency of Chromatin Proteins. In Bacterial Chromatin: Methods in 1914 Molecular Biology, R.T. Dame, ed. (New York, NY: Humana Press).

1915 van der Valk, R.A., Vreede, J., Qin, L., Moolenaar, G.F., Hofmann, A., Goosen, N., and Dame, 1916 R.T. 2017. Mechanism of environmentally driven conformational changes that modulate H-NS 1917 DNA-bridging activity. eLife 6: e27369. doi: 10.7554/eLife.27369.

1918 Vora, T., Hottes, A.K., and Tavazoie, S. 2009. Protein occupancy landscape of a bacterial 1919 genome. Mol Cell 35: 247-253. doi: 10.1016/j.molcel.2009.06.035.

1920 Wang, H., Yehoshua, S., Ali, S.S., Navarre, W.W., and Milstein, J.N. 2014. A biomechanical 1921 mechanism for initiating DNA packaging. Nucleic Acids Res 42: 11921-11927. doi: $1922 \quad 10.1093 /$ nar/gku896.

1923 Will, W.R., Bale, D.H., Reid, P.J., Libby, S.J., and Fang, F.C. 2015. Evolutionary expansion of a 1924 regulatory network by counter-silencing. Nat Commun 5: 5270. doi: 10.1038/ncomms6270. 
1925 Will, W.R., Whitham, P.J., Reid, P.J., and Fang, F.C. 2018. Modulation of H-NS transcriptional

1926 silencing by magnesium. Nucleic Acids Res 46: 5717-5725. doi: 10.1093/nar/gky387.

1927 Wilson, K. 2001. Preparation of Genomic DNA from Bacteria. Curr Protoc Mol Biol 56: 2.4.1-

$1928 \quad$ 2.4.5. doi: 10.1002/0471142727.mb0204s56.

1929 Yamada, H., Yoshida, T., Tanaka, K., Sasakawa, C., and Mizuno, T. 1991. Molecular analysis of

1930 the Escherichia coli has gene encoding a DNA-binding protein, which preferentially recognizes

1931 curved DNA sequences. . Mol. Gen. Genet. 230: 332-336. doi: 10.1007/BF00290685.

1932 Zhou, T., Yang, L., Lu, Y., Dror, I., Dantas Machado, A.C., Ghane, T., Di Felice, R., and Rohs, R.

1933 2013. DNAshape: a method for the high-throughput prediction of DNA structural features on a 1934 genomic scale. Nucleic Acids Res 41: W56-62. doi: 10.1093/nar/gkt437. 